\section{ZOOLOGIQUES, BOTANIQUES, OCÉANOGRAPHIQUES ET GÉOLOGIQQUES ENTEEPRISES $\triangle U X$ INDES NF́ERLANDAISES ORIENTALES en 1899-1900, \\ a bord du SIBOG SOUS LE COMMANDEMENT DE \\ G. F. TYDEMAN PUBLIÉs PAR \\ $M$ A $\mathbf{X}$ W $\mathbf{B} \mathbf{E}$ Chef de l'expédition.}

*I. Introduction et déscription de l'expédition, Max Weber. * II. Le bateau et son équipement scientifique, G. F. Ty d e man. * III. Résultats hydrographiques, G. F. T y de ma n.

*IV. Foraminifera, J. Ho f e $^{1}{ }^{1}$ ).

* IVbis. Xenophyophora, F. E. Schulze.

V. Radiolaria, M. Hartmann.

* VI. Porifera, G. C. J. Vosmaer, M. Burton et I. Ijima ${ }^{1}$. * VII. Hydropolypi, A. Billard ${ }^{1}$ )

* VIII. Stylasterina, S. J. Hicks on et Mlle H. M. England. *IX. Siphonophora, Mlles Lens et $\mathbf{r a n}$ Riemsdijk.

* X. Hydromedusae, $\mathrm{O}$. Ma a s.

* XI. Scyphomedusae, O. M a a s.

* XII. Ctenophora, Mille F. M oser.

*XIII. Gorgonidae, Alcyonidae, J. Verslugs, S. J. Hickson, C. C. Nutting, J. A. Thomson \& Mlle L. M. I. Dean * XIV. Pennatulidae, S. J. Hickson. [et G. Stiasny ${ }^{1}$. *XV. Actiniaria, P. Me Murrieh ${ }^{1}$ ).

*XVI. Madreporaria, A. Álcock, C. J. van der Horst et

*XVII. Antipatharia, A. J. van Peseh. [H. Bosehma')

XVIII. Turbellaria, Sixten Bock.

XIX. Cestodes, (J. W. S pengel †)

*XX. Nema tomorpha, H. F. Nierstrasz.

* XXI. Chaetognatha, G. H. Fo w le r.

*XXII. Nemertini, (A.A. W. Hubrecht $\dagger$ ) et Mme G.Stiasn ${ }^{1}$ ) XXIII. Myzostomidae, R. R. von Stummer.

*XXIV'. Polychaeta errantia, R. Horst, H. A ugener ${ }^{1}$ ).

XXIV2. Polychaeta sedentaria, M. Caullery, F. Mesnil et

* XXV. Gephyrea, C. Ph. Sluiter.

* XXVI. Enteropneusta, J. W. S p engel.

+ XXVIbis. Pterobranchia, S. F. Ha r mer.

*XXVII. Brachiopoda, J. W. Jackson et G. St iasn y.

* XXVIII. Polyzoa, S. F. Ha rmer").

*XXIX. Copepoda, A. Scott at W. H. Leigh-Sharpe.

*XXX. Ostracoda, G. W. M üll er.

* XXXI. Cirripedia, P. P. C. Hoek

*XXXIbis. Rhizocephala. P. N. van Kampon et H. Boschma.

*XXXII. Isopoda, H. F. Nierstrasz et G. A. Brender à

* XXXIII. Amphipoda, J. M. Pirlot.

[Brandis 1 )

* XXXIV. Caprellidae, P. Ma zer.

*XXXV. Stomatopoda, H. J. Ha n sen.

* XXXVI. Cnmacea, W. T. Calman.

* XXXVII. Schizopoda, H. J. Hansen.

*XXXVIII. Sergestidae, H. J. H ansen.

*XXXIX. Decapoda, J. G. de Man, J. E. W. Ihle, J. J. Tesch, H. J. Flipse, Mlle A, J, van Dam et Mlle J.

*XL. Pantopoda, J. C. C. Lo man.

XLI. Halobatidae, D. Ma e Gilla $\mathrm{vr}$ Y.

* XLII. Crinoidea, Ja. Dëderle in et A us tin H. Clark.

* XLIII. Echinoidea, J. C. H. de M e ij ere.

* XLIV. Holothurioidea, C. Ph. Slu i ter.

* XLV. Ophiuroidea, R. Köh le r.

* XLVI. Asteroidea, L. Döderlein et H. Eng el ')

* XLVII. Solenogastres, H. F. Nierstras

* XLVIII. Chitonidae, H. F. Nierstrasz.

*XLIX1. Prosobranchia, M. M. Schepman.

* XLIX2. Prosobranchia parasitica, H. F. Nierstrasz et M. M. * L. Opisthobranchin, R. Bergh. [Schepman * LI. Heteropoda, J.J. T e se h.

* LII. Pteropoda, J. J. Te se h.

* LIII. Lamellibranchiata, P. Pelseneer, Ph. Da utzen ber *LIV. Scaphopoda, Mlle M. Boissevain. [et B. Prashad. LV. Cephalopoda, W. A d a m.

* LVI. Tunicata, C. Ph. Slul t, fet J. E. W. Ihle.

* LVII. Pisces, Max Weber.

- LVIII. Cetacea, Max We ber.

* LIX. Liste des algues, Mme A. Weber.

* LX. Halimeda, Nllle E. S. Barton. (Mme E. S. Gepp).

* LXI. Corallinaceae, Mme A. Weber et M. Foslie.

* LXII. Codiaceae, A. et IIme E. S. Gepp.

LXIII. Dinoflagellata, Coccosphaeridae, (J. P. Lots y + ).

LXIV. Diatomaceae, (J. P. L ot s y $\dagger$ ).

* LXV. Deposita marina, O. B. Böggild.

* LXVI. Résultats géologiques, A. Wi c h ma n n.

\section{Siboga-Expeditie}

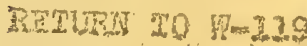

\section{DIR ISOPODEN DER SIBOCA-EXPPDITFON}

IV

\section{ISOPODA GENUINA}

\author{
III. GNATHIIDEA, ANTHURIDEA, VALVIFERA, \\ ASELLOTA, PHREATOCOIDEA
}

voN

\section{H. F, NIERSTRASZ}

UTRECHT

Mit 66 Figuren im Text

Monographie XXXII $d$ de:

\section{UITKOMSTEN OP ZOOLOGISCH,}

\section{BOTANISCH, OCEANOGRAPHISCH EN GEOLOGISCH GEBIED}

verzameld in Nederlandsch Oost-Indië I899-I900

aan boord H. M. Siboga onder commando van

Luitenant ter zee $\mathrm{te}$ kl. G. F. TYDEMAN

UITGEGEVEN DOOR

Dr. MAX WEBER $\dagger$ en Prof. Dr. L. F. DE BEAUFORT Prof. in Amsterdam, Leider der Expeditie

Directeur van het Zoologisch Museum Amsterdam

(met medewerking van de Maatschappij ter bevordering van het Natuurkundig Onderzoek der Nederlandsche Koloniën)

LEIDEN

E. J. BRILL

I94I 

CRUSYRCER IIBRARY SMRTRESORLAN INST.

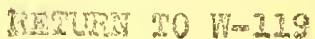




\section{SIBOGA-EXPEDITIE}




\section{Siboga-Expeditie}

\section{UITKOMSTEN}

$\mathrm{OP}$

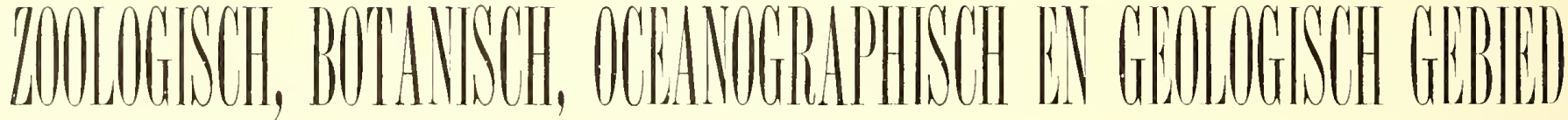

VERZAMELD IN

NEDERLANDSCH OOST-INDIË I 899-I900

A AN BOORD H. M. SIBOGA ONDER COMMANDO VAN

Luitenant ter zee $1^{\mathrm{e}}$ kl. G. F. TYDEMAN

UITGEGEVEN DOOR

Dr. MAX WEBER + en Prof. Dr. L. F. DE BEAUFORT

Prof. in Amsterdam, Leider der Expeditie

Directeur van het Zölogisch Museum Amsterdam

(met medewerking van de Maatschappij ter bevordering van het Natuurkundig

Onderzoek der Nederlandsche Koloniën)

LEIDEN

E. J. BRILL

I94 I 


\title{
DIE ISOPODEN DER SIBOGA-EXPEDITION
}

\author{
IV \\ ISOPODA GENUINA \\ III. GNATHIIDEA, ANTHURIDEA, VALVIFERA, \\ ASELLOTA, PHREATOCOIDEA
}

VON

H. F. NIERSTRASZ

UTRECHT

Mit 66 Figuren im Text

LEIDEN

E. J. BRILL

I 94 I 
Copyright I9tI by E. Ұ. Brill, Leiden, Holland All rights reseried, including the right to translate or to reproduce this book or parts thereof in any form 


\section{ZUM GELEIT}

Als mein verebrter Chef und vaterlicher Freund H. F. NiERSTRASz in September 1937 ablebte, liess er ein grössenteils vollendetes Manuskript über die noch restierenden Gruppen der Siboga-Isopoden nach. Nierstrasz hatte die Absicht diesem Manuskript eine kritische tiergeographische Diskussion über die Verbreitung der Isopoda als Ganzheit beizugeben, wozu er allein durch seinen langjäbrigen Studien berufen war. Diese Diskussion lag aber bei Nierstrasz' Tode nicht vor. Da ich mich nicht kompetent füble diese Lücke auszufüllen, habe ich mich beschränkt zut dem Druckreifmachen der Nierstrasz'schen Arbeit. Nur babe ich die seit Nierstrasz' Tode in 1937 und 1938 erschienenen Arbeiten mit berïcksichtigt und diesbezügliche Einfügungen gemacht. Für tiergeographische Betrachtungen sei besonders auf die interessante Arbeit GurJanova's (78. S. 237-250) bingewiesen. Dieser Autor hat sich ausfübrlich mit der Verbreitung der Isopoden der Bering-See, des Ochotskischen und Japanischen Meeres beschäftigt. Gurjanova weist daraufbin, dass unsere Kenntnisse dieser Gruppe im nördlichen Pazifik zur Zeit noch fragmentarisch ist, sodass die von ihm gegebene Übersicht nur als ein Versuch einer provisorischen Einleitung gelten kann.

Er gibt in seiner Arbeit eine Einteilung der Formen nach der geograpbischen Verbreitung derselben in Zusammenhang mit der Umwelt und weist daraufbin, dass die „Verbreitung der Isopodenfauna in den 3 Meeren zum grössten Teil durch die Bodenbeschaffenbeit und das Relief, so wie durch Strömungen bedingt ist. Nicht minder wichtig ist auch die geographische Lage der Meere: der Unterschied in der Entfernung vom Äquator zwischen dem Japanischen, Ochotskischen und Beringsmeere”. Das Bering-Meer enthält bauptsächlich Kaltwasserformen. Hier sind vor allem die Asellota reich vertreten, die mebr südlich, z.B. im Japanischen Meere von warmwasser liebenden Sphaeromidae vertreten sind". Das Japanische Meer weist eine grössere Mannigfaltigkeit an Formen auf als die nördlichen Meere. Dort ist die Warmwasserfauna des oberen Sublitorals sebr reich, während grössere Tiefen von einer an Artenzabl armen, gemässigten Fauna bewobnt werden. In dem Bering-Meer ist die isopodenfauna sowobl in den oberen Schichten, als auch im Abyssal artenreich, wäbrend im Ochotskischen Meere die reiche Flachwasserfauna wiederum von der ärmeren, aber sebr eigentümlichen Tiefenfauna absticht". Dies hängt wie Gurjanova hervorbebt wiederum zusammen mit der Unterschichtung bzw. Oberschichtung von $W$ asser, dass durch $W$ asserströmungen in den verschiedenen Meeren gebracht wird. In den nördlichen Meeren schichtet sich wärmeres $W$ asser, das vom Süden her kommt über die kältere Tiefsee, während auch kaltes Tiefenwasser vom Norden nach dem Süden durchdringt.

Es wäre vielversprechend die tropischen Verbältnisse mit den Verbältnissen der nördlichen Meere zu vergleichen. Ich will darauf aber aus dem oben erwähnten Grïnden verzichten, werde nur bei den verschiedenen Arten und Gattungen auf die von GuRjanova gemachten Bemerkungen zurückgreifen und einige erläuternde Bemerkungen dieses Autors zufügen zu dem Manuskripte Nierstrasz, das im übrigen ganz und gar seine Arbeit bleibt.

Utrecht, 15 Juni 1941

J. H. SCHUURMANS STEKHOVEN Jr. 


\section{IV \\ ISOPODA GENUINA}

\section{VOR WORT}

Bis jetzt sind von den Siboga-Isopoden die Isopoda Chelifera (126) und von den Isopoda Genuina die Unterordnungen der Epicaridea (131) und der Flabellifera (130) beschrieben worden. Ich möchte hierbei bemerken, dass es rationeller gewesen wäre die Isopoda Chelifera vollständig von den Isopoda Genuina zu trennen und beide als gesonderte Ordnungen zu betrachten, sodass wir besser sprechen könnten von den Ordnungen der Anisopoda oder Tanaidacea und der Isopoda. Folgen wir die Einteilung Calman's (27) mit Verbesserungen von MONOD (111), so kommen wir zu folgenden Subordnungen der Isopoden: Flabellifera, Valvifera, Asellota, Pbreatocoidea, Anthuroidea, Gnatbiidea, Epicaridea und Oniscoidea; von diesen sind die Flabellifera und Epicaridea schon erschienen und es bleiben also die Valvifera, Asellota, Phreatocoidea, Anthuroidea, Gnathiidea übrig, denn die Oniscoidea sind im Material der Siboga nicht vorhanden. Leider ist dies auch der Fall mit den Phreatocoidea und den Gnathiidea; ich habe jedoch diese beiden Unterordnungen doch aufgenommen und die Oniscoidea als echte Landtiere fortgelassen. Freilich aber sind die übrigen Unterordnungen auch nur spärlich vertreten, vor allem die Asellota. Die Gruppen sind in der sonst üblichen Weise vorgeführt.

\section{Unterordnung GNATHIIDEA}

Gnathia Leach

1 Gnathia philogona Monod.

Strasse von Malacca, Singapur (113, S. 423).

2 Gnatbia perimulica Monod.

Golf von Siam: Koh Mesan und Ko Chang, 2-27 m (113, S. 425).

3 Gnatbia alces Monod.

Golf von Siam, zwischen Koh Mesan und Kap Liant, 9-14 m; Singapur (113, S. 495, 496).

4 Gnatbia mortenseni Monod.

Golf von Siam an verschiedenen Stellen, 18-54 m (113, S. 501).

5 Gnatbia pilosipes Monod.

Golf von Siam. Koh Kam und Koh Kahdal, 7-9 m (113, S. 446). 
6 Gnatbia coralliopbila Monod.

Golf von Siam, Koh Chang, 2 m (113, S. 395).

7 Gnatbia consobrina Monod.

Nagasaki, Japan (113, S. 449).

8 Gnathia nipponensis Monod. $34^{\circ} 20^{\prime} \mathrm{N} .130^{\circ} 10^{\prime}$ O., $108 \mathrm{~m}(113$, S. 381$)$.

9 Gnathia rectifrons Gurjanova.

Japanisches Meer, Bai des Peter des Grossen, Tiefe $80-88$ m (78, S. 230; 77, S. 259).

10 Gnathia schmidti Gurjanova.

Japanisches Meer, Wladimir Bai, Tiefe 308-1002 m (77, S. 259, 78, S. 232).

11 Gnatbia derzhavini Gurjanova.

Japanisches Meer, Tiefe 110-121 m (78, S. 233, 77, S. 259).

12 Gnatbia tuberculata Richardson.

Japan, zwischen Nanao, Hondo und Isuruga, Hondo, $37^{\circ} 22^{\prime} 30^{\prime \prime}$ N., $137^{\circ} 10^{\prime}$ O., Tiefe $1124 \mathrm{~m}$ (155, S. 76) Confer (78, S. 230) im Bai des Peter des Grossen, Tiefe 892 m (78, S. 245).

13 Gnatbia latidens (Beddard).

Flinder's Passage, N.O. Australien, 13 m (14, S. 120).

14 Gnathia calamitosa Monod.

Disaster Bay, Neu-Süd-Wales, 54-72 m; $37^{\circ} 05^{\prime}$ S. $150^{\circ} 05^{\prime}$ O., 54-90 m (113, S. 508).

15 Gnathia calmani Monod.

„Victoria-Tasmania Cables” (113, S. 552).

16 Gnathia pustulosa Hale.

Adelaide, Süd-Australien (79, S. 5).

Glenelg, Süd-Australien (84, S. 244).

17 Gnatbia mulieraria Hale.

Golf von St. Vincent, Süd Australien, 13-25 m (79, S. 4, 84, S. 243).

18 Gnathia regalis Monod.

Three Kings, Neu-Seeland, 117 m (113, S. 536).

19 Gnathia akaroensis Monod.

Akaroa, Neu-Seeland, 10 m (113, S. 443).

20 Gnatbia pacifica Monod.

Colville Channel, Neu-Seeland, 63 m (113, S. 451).

21 Gnathia polythrix Monod.

Three Kings-Inseln, Neu-Seeland, 118 m (113, S. 371).

22 Gnatbia bracbyuropus Monod.

Akaroa und Lyttelton, Neu-Seeland; bis $11 \mathrm{~m}$ (113, S. 580).

Nur die Praniza-Form ist bekannt. 
23 Gnathia aureola (Stebbing).

Lifu, Loyalty-Inseln, Bai von Sandal; Lagune von Mangareva, Gambier-Inseln; Küste Natal's (113, S. 573; 9, S. 385).

Nur die Praniza-Form ist bekannt.

24 Gnathia virido-nitens Monod.

Mangareva, Cambier-Inseln. (113, S. 576).

Nur die Praniza-Form ist bekannt.

25 Gnathia margaritarum Monod.

Golf von Panama, Pontadara, Pontadora und Iles des Perles. 14- $47 \mathrm{~m}(113$, S. 439).

26 Gnatbia elongata (Kröyer).

Japanisches Meer, 120-3000 m (78, S. 235, 77, S. 259), Strasse von Georgien, Britisch Kolumbien 45-360 m, Korea(?) weiter nord-atlantisch und arktisch, Tiefe von 2-927 m, von W.-Grönland, Jan Mayen, Kara-Meer, New York (113, S. 354, weiter von O. Grönland, Tiefe $60 \mathrm{~m}$ (137, S. 4), Island, $40-130 \mathrm{~m}(200$, S. 14).

G. elongata ist nach GURJANOva (78, S. 245) eine autochthone, arktische Form, die aus dem Eismeer in den Stillen Ozean eindringt. Dort kommt sie nur in Tiefen von $76-900 \mathrm{~m}$ vor.

27 Gnathia crenulatifrons Monod.

Kalifornien, La Jolla, 18-45 m (113, S. 393).

28 Gnathia taprobanensis Monod.

Trincomalı, Ceylon, $13 \mathrm{~m}$ (113, S. 504).

29 Gnatbia antarctica (Studer).

Kerguelen; Prince Edward-Insel: Süd-Georgien; weiter antarktisch; nördlich bis Patagonien und Chili, südlich bei dem Ross-Meer; Tiefe bis 457 m (113, S. 344).

Die Varietät insularis VANHÖFfen im Praniza-Stadium bei Kerguelen (218, S. 487).

30 Gnatbia tuberculosa (Beddard).

Kerguelen, 54-229 m (113, S. 377).

31 Gnatbia rhinobatis Kossmann.

Rotes Meer (103, S. 105, 119, S. 247).

32 Gnatbia disjuncta Barnard.

Knysna Heads, Süd-Afrika, 72 m (7, S. 335).

33 Gnathia spongicola Barnard.

Table Mountain; Cape Point; Lion's Head, alle Süd-Afrika; $230-342$ m (7, S. 334).

Die var. minor Barnard: Buffel's Bay, 54 m (7, S. 334).

34 Gnatbia africana Barnard.

St. James, False Bay; Sea Point; beide Süd-Afrika (4, S. 202; 6, S. 334a); Port Alexander, Angola, $72 \mathrm{~m}(196$, S. 1).

35 Gnathia cryptopais Barnard.

Duminy Point, Salandanha-Bai, 157 m (7, p. 418).

Überdies gibt es noch etwa 28 atlantische und arktische Arten. Obengenannte Liste habe 
ich zusammengesetzt mit Hilfe von MoNoD's brillanter Beschreibung der Gnatbiidae (113). Es ergibt sich hieraus, dass Arten aus dem Indo-australischen Archipel und den Philippinen noch nicht beschrieben worden sind Aber MONOD gibt eine lange Liste von undeterminierbaren Formen, aus welcher sich ergibt, dass bei Batavia, Java, Borneo, den Philippinen jedoch Arten der Gnathiiden vorkommen (113, S. 605); weiter im Roten Meer und bei der Insel Aldabra (Comoren).

Die Arten Gnatbia tuberculata, Gn. derzhavini, Gn. schmidti, Gn. rectifrons und Elaphognathia monodi (Confer S. 236, 238), gehören zu den endemischen Formen des Japanischen Meeres. Von diesen kommen Gnathia tuberculata und Gn. rectifrons in dem Peter des Grossen Bai vor (78, S. 245).

\section{Euneognathia Stebbing}

1 Euneognatbia gigas (Beddard).

Bai von Cumberland, Christmas Harbour, Kerguelen, 229 m; weiter antarktisch (205, S. 247; 113, S. 319).

Die einzig bekannte Art.

\section{Elaphognathia Monod}

1 Elaphognathia rangifer Monod.

Singapur; zwischen Koh Mesan und Kap Liant, 14 m (113, S. 571).

2 Elaphognatbia ferox (Haswell).

Port Jackson (93, S. 1006).

3 Elaphognatbia lucanoides Monod.

Misaki, Japan, 5 m (113, S. 568).

4 E. monodi Gurjanova.

Japanisches Meer, $60 \mathrm{~m}, 0-2 \mathrm{~m}$ (77, S. 256; 78, S. 236).

Elaphognathia monodi ist eine endemische Form des Japanischen Meeres(78, S. 245).

5 Elaphognathia insolita (Stebbing).

Karativo, Golf van Manaar (188, S. 9).

\section{Thaumastognathia Monod}

\section{Thaumastognathia diceros Monod.}

Three Kings, Neu-Seeland, 117 m (26, S. 307).

Die einzig bekannte Art.

Die Gattungen Akidognathia STebing, Bathygnathia Dollfus, Paragnathia Omer CooPER und Perignathia MONOD enthalten keine indopazifische Formen. 


\section{Unterordnung ANTHURIDEA}

\section{Familie AnthuridaE}

\section{Anthura Leach}

\section{Anthura gracilis (Mont).}

Kerguelen, 9-18 m (65, S. 244). übrigens atlantisch und mediterran. Ob der Fundort Kerguelen richtig ist, lässt sich bezweifeln, BARNARD nennt ihn wenigstens nicht (8, S. 130).

Andere Arten kommen nicht vor.

\section{Ptilanthura Harger}

Von der mit Antbura verwandten Gattung Ptilanthura Harger kommt nur die einzige Art Ptilanthura tenuis Harger an der Ost-Küste Nord-Amerika's vor, Tiefe 0-34 m (149, S. 67).

\section{Exanthura Barnard}

1 Exanthura macruron Barnard.

Seapoint bei Kapstadt (6, S. 338a).

2 Exanthura filiformis (Lucas).

Kap. St. Blaize, 225 m; Cape Infanta, 77 m; Lion's Head bei Kapstadt, 171-414 m; Algerien, 225450 m (7, S. 342; 8, S. 131, 9, S. 388).

Keine anderen Arten sind bekannt.

\section{Haliophasma Haswell}

1 Haliophasma purpureum Haswell.

Port Jackson (91, S. 477).

2 Haliophasma tricarinatum Barnard.

Agulhas-Bank, 72 m; Kap.; St. Blaize, 72-76 m (8, S. 132; 9, S. 386).

3 Haliophasma coronicauda Barnard.

Saldanha-Bai, Süd-Afrika, 157 m (9, S. 388).

Die vierte Art, Haliophasma dakarense, Barnard ist bis Dakar, West-Afrika, 21-25 m. gefunden worden (8, S. 133). Die Gattung zeigt, mit Ausnahme von purpureum, eine atlantische Tendenz.

\section{Notanthura Monod}

Die Gattung Notanthura Monod mit der einzigen Art Notantbura barnardi ist atlantisch (Bai von Douala, Souelaba, Kamerun, 115, S. 209, 117, S. 17). Indessen ist es zweifelhaft, ob die Gattung an dieser Stelle gesetzt werden darf. 


\section{Eisothistos Haswell}

1 Eisothistos vermiformis Haswell.

Watson's Bay, Neu-Süd-Wales, litoral (94, S. 676).

Weiter beschrieb VANHÖFFEN noch' zwei andere Arten:

E. atlanticus von Porto grande, St. Vincent, Kap Verdische Inseln, nach Barnard (8, S. 134) auch

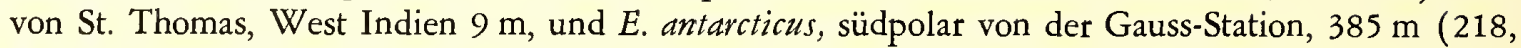
S. 494); leztere Form wird auch von TatTersall erwähnt von $77^{\circ} 5^{\prime}$ S. $164^{\circ} 17^{\prime}$ O., $252 \mathrm{~m}$ (205, S. 232).

\section{Malacanthura Barnard}

1 Malacantbura linguicauda Barnard.

Umhlangakulu River, Natal, 90 m (7, p. 339); Lion's Head, 171 m (8, S. 133; 9, S. 388).

Die einzig bekannte Art.

\section{Anthelura Norman et Stebbing}

1 Anthelura remipes Barnard.

Lion's Head bei Kapstadt, 281 m (6, S. 340a).

Die fünf übrigen Arten sind atlantisch.

\section{Xenanthura Barnard}

1 Xenanthura orientalis Barnard.

Vatta Kayal. Aleppey (11, S. 307).

Die zweite Art brevitelson Barnard ist von BARNARD von St. Thomas, West-Indien, 45$50 \mathrm{~m}$. beschrieben worden (8, S. 139).

\section{Ananthura Barnard}

Die Gattung Ananthura BarNard zeigt zwei Nord-Atlantische und eine mediterrane Art (8, S. 136).

\section{Hyssura Norman and Stebbing}

Die Gattung Hyssura Norman \& Stebbing, mit 2 Arten, ist Atlantisch (8, S. 137).

\section{Cyathura Norman et Stebbing}

1 Cyathura indica Barnard.

Singapore, Siam und Paumben; Adam's Bridge, Ceylon, (1-25 m, 8, S. 141); Quilon, Travancore $(11$, S. 306).

2 Cyathura siamensis Barnard.

Siam, 5-8 m (8, S. 140). 
3 Cyatbura pusilla Stebbing.

Ceylon (186, S. 9); Wasin, Ost-Afrika, 18 m (191, S. 91).

4 Cyatbura carinata (Kröyer).

China (8, S. 140) Ochotskisches Meer, Tiefe $3 \mathrm{~m}$ (78, S. 126; 77, S. 259), Buffalo River, East London; Zwartkops River, Algoa Bai (6, S. 336a); weiter nord-atlantisch und mediterran, bis $35 \mathrm{~m}$; auch im baltischen Meer (105, S. 172). Die Art hat eine nahezu kosmopolitische Verbreitung und ist mehr wärmeliebend.

Die fünfte Art, C. crucis Barnard, kommt vor in West-Indien (8, S. 141).

\section{Apanthura Stebbing}

1 Apantbura sandalensis Stebbing.

Tirunayamkudam, Vembanad Lake, Travancore (11, S. 306); Chilka Lake (46, S. 881); Sandal Baym

Lifu, Loyalty-Inseln (183, S. 622); St. James, False Bay (8, S. 342a).

2 Apantbura coppingeri Barnard.

Dundas Straits, Nord-Australien, $31 \mathrm{~m}$ (8, S. 142).

3 Apanthura xenocheir Stebbing.

Egmont-Riff, Seychellen (191, S. 95).

4 Apanthura africana Barnard.

Paternoster Point, Saldanha-Bai, 144 m (6, S. 342a).

Es gibt noch eine atlantische Art, $A$. senegalensis Barnard, von Dakar, 9 m.

\section{Panathura Barnard}

1 Panathura serricauda (Barnard).

Sea Point bei Kapstadt; St. James und Buffel's Bay, False Bay (7, S. 340).

Die einzig bekannte Art.

\section{Mesanthura Barnard}

1 Mesanthura albolineata Barnard.

Singapore (8, S. 144).

2 Mesanthura ocellata Barnard.

Siam, 2 m (8, S. 144).

3 Mesanthura maculata (Haswell).

Port Jackson, Port Stephens, Griffith Point, Victoria (92, S. 306); Botany Bay, 90-94 m (92, S. 216); Süd-Australien (84, S. 245); Lyttelton Harbour, Neu-Seeland, 0-94 m (30, S. 72; 216, S. $152 ; 8$, S. 144). 
4 Mesantbura catenula (Stimpson).

Kalk-Bai und St. James, False Bay; Sea Point, Simon’s Bay; alle Süd-Afrika (6, S. 345a; 92, S. 420).

Die fünfte Art, pulchra Barnard, ist atlantisch.

Die verwandte Gattung Skuphonura BARNARd zeigt nur die einzige Art Skuphonura latipes Barnard aus West-Indien, 7-36 m, (8, S. 145). Ebenso Kuppellonura BARNARD mit der einzigen Art Kuppellonura mediterranea BARNARD, von Messina (8, S. 146).

\section{Accalathura Barnard}

1 Accalathura gigas (Whitelegge).

Arakan, Unter-Burma, 23 m (12, S. 1481); Port Jackson, 65-70 m (223, S. 229); Golf von St. Vincent (8, S. 148); Süd-Australien (84, S. 246); Cargados Carajos, $50 \mathrm{~m}$ en Saya de Malha, $47 \mathrm{~m}$, Seychellen (191, S. 93).

2 Accalathura borradailei (Stebbing).

Siam, 24 m (8, S. 149); Fadifolu Atoll, Maldiven, 43 m (187, S. 701); Chilka Lake, bis 5 m (46, S. 881).

Die beide andren bekannten Arten, A. crenulata (Richardson) und crassa Barnard sind atlantisch.

\section{Leptanthura G. O. Sars}

1 Leptanthura orientalis Barnard.

Singapore (8, p. 150).

2 Leptanthura australis (Haswell).

Port Jackson (91, S. 478); Dundas-Strasse, Nord-Australien, 31 m (109, S. 311).

3 Leptanthura crassicornis (Haswell).

Port Jackson (91, S. 478); vielleicht auch im Mondragon Paar und Chilair Paar, Ceylon 16-20 m $(188$, S. 8).

4 Leptanthura dienenensis (Haswell).

Hobart, Tasmanien (93, S. 1011).

5 Leptanthura chiltoni (Beddard). $37^{\circ} 34^{\prime}$ S. $179^{\circ} 22^{\prime}$ O., $1260 \mathrm{~m}(13$, S. 144$)$.

6 Leptanthura laevigata (Stimpson).

Algoa-Bai, 45 m; East London, 58 m; Umhlangakulu River, 90 m; Cove Rock, 76 m; Keiskamma Point, 60 m, alle Süd-Afrika (6, S. 347a); Simons Town (218, p. 492).

Von den übrigen Arten sind 4, n.l. tenuis (G. O. SARs), affinis (BONNIER), thori BARNARD und truncata RiCHARDSON, atlantisch; überdies gibt es noch eine antarktische Art, glacialis HodGSON (98, S. 9, 205, S. 232). 


\section{Calathura Norman et Stebbing}

\section{Calathura branchiata (STIMSSON).}

Ochotskisches Meer (78, S. 130) Tiefe 335-1076 m; Bering-Meer (78, S. 130; 77, S. 260) Tiefe $108-360 \mathrm{~m}$, arktische Meere der URSS (75, S. 31-34) $77^{\circ} 14^{\prime}-79^{\circ} 25^{\prime} \mathrm{N}$., $70^{\circ}-105^{\circ} 38^{\prime} \mathrm{O}$., Tiefe 43-475 m. Die Art ist nord-atlantisch und arktisch (149, S. 72) Grönland (137, S. 3) Tiefe 30-400 m, Island (200, S. 9). Tiefe 225-367 m; Grösste Tiefe 1500 m.

Die einzige Art.

C. brachiata gehört zu den arktischen Formen, die von den arktischen Gewässern im Bering-Meer eindringen. Man findet sie auch in dem Ochotskischen Meer.

\section{Katanthura nov. gen.}

Mundteile zum Durchbohren und Saugen eingerichtet.

Keine Statocysten. Exopod der Uropoden nicht reduziert.

Fünftes Glied der Pereiopoden nicht „underriding” das sechste. Maxilliped 4-gliedrig.

Diese Gattungs-Diagnose habe ich aus praktischen Gründen ganz im Stile BARNARD's (8, S. 129) gehalten. Denn BARnARD ist der einzige, welcher versucht hat Ordnung in die Familie der Anthuriden zu schaffen und zwar mit Erfolg.

\section{Katanthura barnardi nov. spec. (Fig. $1-13$ ).}

Stat. 305, Solor-Strasse, 113 m. 1 Exemplar, . .

Länge $14 \mathrm{~mm}$. Farbe des Cephalons und der Tharakomeren II und III hellbraun; übrige Teile des Körpers dunkelbraun. Alle Thorakomeren sind ungefähr gleich lang, mit Ausnahme des achten, welches viel kürzer ist und das erste Pleomer umfasst; dieses Pleomer ist kleiner als die Pleomere

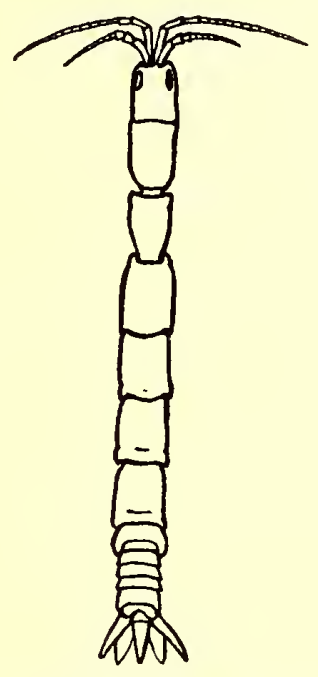

Fig. 1. Katantbura barnardi $\circ, \pm 4 \times$.

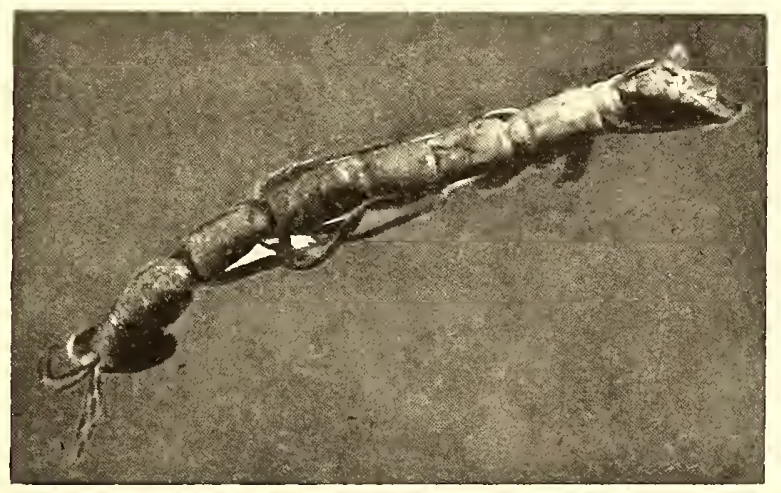

Fig. 2. Katantbura barnardi 오 $\pm 41 / 2 \times$.

II-IV und vor allem als das viel längere Pleomer V (Fig. 1). Augen gross, seitlich. Die Antennae reichen ungefähr bis an den Hinterrand des dritten Thorakomers; die Antennulae sind viel kürzer. Caudal vom fünften Pleomer folgt ein dreieckiges Stück (freier Rest des sechsten Pleomers?), an den Seiten mit 2 kugelförmigen Stücken und das zungenförmige Telson (Fig. 1). Oostegiten fehlen. 
Die Antennula zeigt 3 pedunculäre Glieder und ein Flagellum mit 14 Gliedern, von welchen das zweite sehr lang is (Fig. 3).

Antenna: Flagellum mit 22 Gliedern (Fig. 4).

Labrum gross, dreieckig (Fig. 5).

Mundteile. Über den Bau der Mundteile sind die Angaben sehr spärlich. Wir haben nur die schönen Untersuchungen Schrödte's über Anthura und Norman und Stebirng's (138, S. 120) über Antbura, Cyathura, Anthelura, Hyssura, Paranthura und Calathura, aber von diesen Gat-

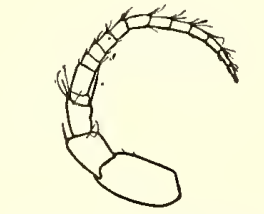

Fig. 3. Katanthura barnardi Antennula, $23 \times$.

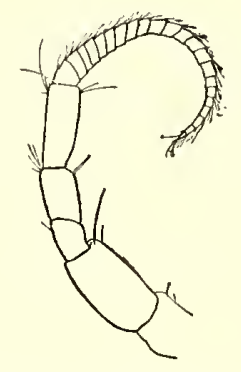

Fig. 4. Kathantura barnardi Antenna, $23 \times$

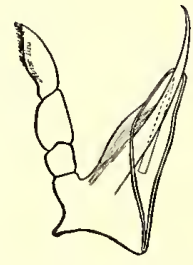

Fig. 5. Katanthura barnardi, rechte Mandibula, $43 \times$.

tungen gehören nur letztgenannte zwei zu BARNARD's Section B, zu welcher Katanthura ebenso sicher zu rechnen ist. Weiter noch einiges bei DohrN über Paranthura costana Bate et Westwood (59, S. 83), bei Chilton über Cruregens fontanus Chilton (30, S. 175), Miss Sexton (175. S. 241) und HANSEN (88, S. 191). Der Bau ist sehr kompliziert und schwierig zu verstehen, was auch HANSEN zugibt (88, S. 180). Von meinem einzigen Exemplar habe ich die Mundteile zum Teil isolieren können und kann von der Struktur etwas geben.

Mandibula (rechte, Fig. 5). Von andrem Bau als von Norman und Stebbing für die Sektion $\mathrm{B}$ angegeben worden ist $(138, \mathrm{~S} .120,122)$. Sie ist hohl und an der medialen Seite offen; in der Höhle befindet sich eine grosse Stachel, welche oral herausragt und an welche sich wenigstens 1 Muskel anheftet. Proximal von dieser Stachel bildet sich eine zweite kleinere, wahrscheinlich als ReserveStachel. Norman und Stebirng schrieben (138, S. 122): ,the mandible forms an acutely-pointed lancetlike organ and the saw-like process characteristic of the genera of Section $A$ is here represented by the expanding lobes of the base of the lancet, which form a channel through which, when the incision has been made, the liquid may be sucked." Diese Beschreibung trifft für unsre Form nicht zu; Norman und Stebbing's ,lancet-like organ” ist eine freie Nadel, welche wahrscheinlich aus- und eingezogen werden kann; sie macht aber keinen Teil der Mandibula selbst aus, sondern ist ein ganz freies Gebilde, welches ersetzt werden kann. Der Bau der Mandibula ist sicher viel komplizierter, als man bis jetzt annimmt. Nachuntersuchungen an vielen Exemplaren von derselben Art sind erwünscht. Der Palp ist 3-gliedrig; das dritte Glied trägt an der lateralen Seite zwei Reihen von Stacheiln. Norman und Stebbing umschreiben die Maxillula ihrer Section B in dieser Weise: „First maxillae take the form of a greatly produced, very narrow, spear-like organ, which towards the point is channelled on one side and finely striated at the margins, this constituting an admirably adapted instrument for deeply probing the wound where the lancet-like and more cutting mandible has first made the incision" (138, S. 122). Für unsre Form trifft diese Beschreibung gar nicht zu. Die Maxil- 
lula (Fig. 6) ist nicht ein einfaches ,spearlike organ”, sondern sie ist röhrenförmig und produziert freie Stacheln, von welchen eine hervorragt und zwei andere - eine ausgebildete und eine junge sich im Inneren befinden. Es macht auch hier den Eindruck, dass die hervorragende Stachel aus- und eingezogen werden kann. Ebensowenig als bei der Mandibula lässt sich bei der Maxillula einsehen, wie diese Organe morphologisch zu verstehen sind. Auch für die Maxillula sind Nachuntersuchungen an einem reichen Material sehr nötig. Maxilliped (Fig. 7). Diese zeigen 2 Basalstücke, beide unpaarig.

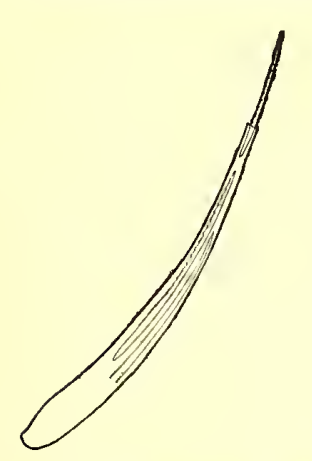

Fig. 6. Katantbura bar nardi. Maxillula, $47 \times$. Das distale trägt ein rundes Epipod; es folgen dann 3 Glieder, von welchen das erste lang ist und ein medialen Fortsatz trägt, welcher rechts durch eine Naht getrennt wird. Das zweite, welches einige lange Haaren am medialen Rand trägt, und das dritte - mit einer terminalen Büschel von Haaren sind viel kürzer. Wie sind diese Komponente aufzufassen? Die Litteratur über die Maxillipeden der Anthuriden ist sehr dürftig. Norman und Stebbing bilden dieses Organ ab bei Calathura brachiata (Stimpson) und Cyathura carinata (Kröyer) (138, Taf. 26, Fig. 1 mxp; Taf. 27, Fig. III mxp); die Verhältnisse bei erstgenannter Form stimmen mit denen unsrer Form überein; es ist aber nur ein Basalstück vorhanden. Der Zustand bei Cyatbura carinata ist ungefähr derselbe; nur fehlt hier der mediale Fortsatz. Norman und Stebbing sagen aber nichts über den morphologischen Wert der verschiedenen Teilen. BARNARD beschreibt die Maxillipeden und bildet sie bei vielen Formen ab

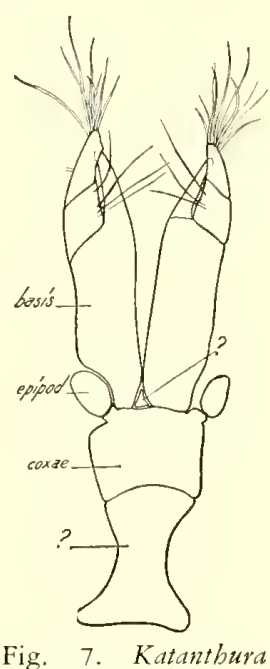
barnardi, Maxilliped (Thorakopod I),

$$
47 \times \text {. }
$$
(8, S. 112 und 113). Unglücklicherweise hat er das „,basal joint, which is anchylosed to the head and which is ralely found in microscopical preparations" nicht abgebildet. Seine Figuren sind deshalb unvollständig und wir können sie daher nur mit Vorsichtigkeit benutzen. Versuchen wir aber die Verhältnisse bei unsrer Form klar zu machen. HANSEN gibt ein Übersicht über den Bau der Maxillipeden der Isopoden (89, S. 119), in welcher aber von den Anthuriden nichts gesagt wird. Weiter beschreibt Hansen noch die Maxillipeden von Anthelura truncata (Hansen) (88, S. 181 und 182; Taf. XV, Fig. $2 \mathrm{~g}$ ); sie ist 5-gliedrig. Das erste Glied ist sehr klein und trägt ein Epipod; dieses Glied muss deshalb, in Übereinstimmung mit dem, was Hansen später beschrieben hat (89, S. 120, Taf. VII, Fig. 3c, Ga und 7a) als Coxa angedeutet werden. Das zweite Glied ist deshalb die Basis; leider besitzt diese keinen Fortsatz. Die Glieder III-V bilden also zusammen das Endopod. Andere Arten von Anthelura sind A. elongata Norman et Stebbing und A. remipes Barnard. Von diesen soll das Maxilliped von elongata einen Fortsatz an der Basis haben (vide BARnard 8, S. 135; in der Beschreibung Norman und Stebbing's kann ich nichts hierüber finden, auch nicht in ihren Figuren). Dasselbe ist der Fall bei remipes (Barnard, Taf. 28, Fig. B, mxp). Das distale der beiden basalen Stücken bei unsrer Form muss deshalb als die verwachsenen Coxae angesehen werden, denn es trägt die Epipoden; solch eine Verwachsung habe ich nirgends in der Litteratur gefunden, ausser bei NormaN und Stebbing in ihrer Figur III mxp, Taf. 27, bei Cyathura carinata (Kröyer) es fehlen aber bei dieser Form sowohl der innere Fortsatz wie das Epipod; diese sind wenigstens nicht abgebildet worden. BARNARD, vermeldet für Xenanthura, dass die zweiten Glieder (die Bases) miteinander verwachsen sein sollen (8, S. 113); dies ist also ein andrer Fall. Zwischen beiden Bases unsrer Form findet man noch ein kleines dreieckiges Stückchen, dessen Deutung mir fremd bleibt. 
Es bleibt noch das proximale Stück der beiden Basalstücke übrig. Man könnte hier an verwachsenen Praecoxae denken; diese Möglichkeit entbehrt aber aller Wahrscheinlichkeit. Denn erstens ist eine Praecoxa bei den Isopoden nur bei sehr wenigen Formen von Hansen gefunden worden, unter welchen sich keine Anthuriden befinden (89, S. 120), obwohl HANSEN die Möglich-

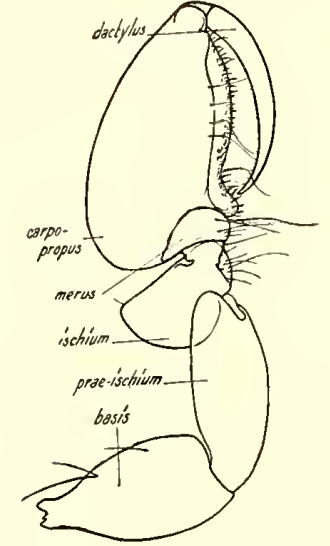

Fig. 8. Katantbura barnardi, Thoracopod II (Gnathopod I), $23 \times$ keit äussert, dass sie bei verschiedenen anderen Formen auch vorkommen soll; zweitens aber ist die Praecoxa immer sehr klein und unansehnlich und ist von Verwachsung keine Rede, während beide Praecoxae in unsrem Fall zu einer sehr grossen Platte verwachsen sein könnten. Vielmehr nehme ich an, dass das proximale Basalstück zum Cephalon gehört und also nicht zum Maxilliped; dieses soll also aus Coxa, Basis und zwei Endopod-Gliedern bestehen, also 4-gliedrig sein.

Thorakopod II (Gnathopod I) : sehr stark entwickelt (Fig. 8). BARNARD behauptet, dass das sechste Glied, von ihm Propodus genannt (C 8, S. 113), nach HANSEN der Carpo-propus (89, S. 121), beinahe immer subchelat sein soll; dies ist hier nicht der Fall, aber wohl bildet dieses Glied mit dem Dactylus eine sehr starke Zange. Auch soll nach BArnard der Dactylus immer eine kurze Klaue tragen bei den Formen der Section B nach Norman und StebBing (138, S. 128), zu welcher unsre Form sicher zu rechnen ist; dies gilt aber hier nicht, denn eine freie Klaue fehlt überhaupt.

Thorakopod III (Gnathopod II). Die „Hand” ist weniger stark als bei Gnathopod I, aber der Carpo-propus ist viel stärker bewaffnet (Fig. 9). Der Dactylus trägt eine kleine Klaue. Thorakopod IV (Gnathopod III) wieder schwächer als die vorhergehenden (Fig. 10). Der Dactylus ist aber viel grösser und die sehr kleine Klaue befindet sich im Dactylus, dessen Spitze eine Öffnung zeigt, durch welche die Klaue hinein- und hinausgezogen werden kann, was wenigstens wohl wahrscheinlich ist. Dieser Zustand ist, so weit ich es beurteilen kann, noch nie bei den Isopoden beobachtet worden.

Thorakopoden V---VIII (Pereiopoden I-IV). Alle etwa gleich lang (Fig. 11). BarNARd (8, S. 113) beschreibt diese Extremitäten ausführlich und weist auf die Besonderheit, dass das fünfte Glied (er muss hiermit den Merus meinen) cylindrisch und ungefähr ebenso lang wie das sechste (Carpo-propus) sein kann, oder sehr kurz, dreieckig und ,underriding” das sechste Glied. Der Habitus des Merus hat deshalb klassifikatorischen Wert. BARNARD sagt, dass es nicht schwer fällt zu. entscheiden, ob dieses ,underriding” statt findet oder nicht (8, S. 114).

Ich kann dies nicht einsehen. Vergleicht man z.B. in seiner Figur 2 die Abbildung von Ananthura (B) mit der von Kupellonura (C) so sieht man, dass die Unterschiede unbedeutend sind; dennoch ist Ananthura nicht und Kupellonura wohl ,unterriding”! Nur in extremen Fällen lässt sich dieses Merkmal anwenden. In unsrem Fall kann aber kein Zweifel vorhanden sein; Katanthura ist „not underriding”. Die Klaue des Dactylus ist kurz.

Das Metasoma (Fig. 12) zeigt 5 freie Pleomere, von denen I kurz und schmal ist und vom achten Thorakomer umfasst wird, II, III und IV sind gleich lang, V is länger. Die Pleopoden (Fig. 13) sind einander gleich und ungefähr gleich gross; das erse Paar is nicht operculiform, gleich wie bei Hyssura, Xenanthura und Kupellonura. Das Exopod ist immer breiter und länger als das Endopod; beide Poden tragen einen starken apicalen Bündel von langen Haaren. Das Exopod ist transparant, während das Endopod undurchscheinend und etwas dicker und iiberdies etwas runzelig ist und kleine 
Flecken zeigt. Das Pleotelson besteht aus einem dreieckigen Teil, an welchen sich links und rechts ein rundes kugelförmiges Stück mit Einbuchtung anschliesst, und einen zungenförmigen, langen Fortsatz. Der Dreieck ist wahrscheinlich das sechste Pleomer, der zungenförmige Teil das Telson

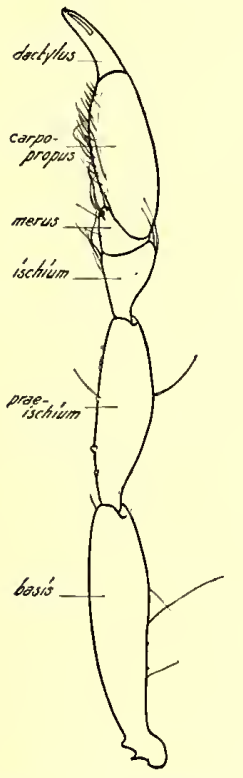

Fig. 9. Katanthura barnardi, Thorakopod IV (Gnathopod III), $23 \times$.

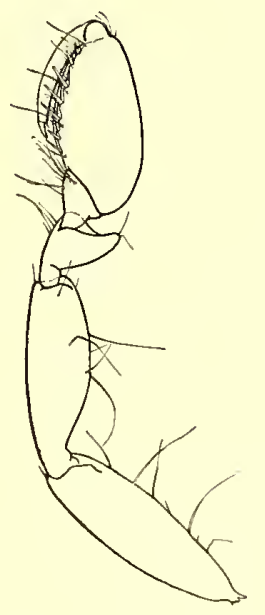

Fig. 10. Katantbura barnardi, Thorakopod III (Gnathopod II), $23 \times$

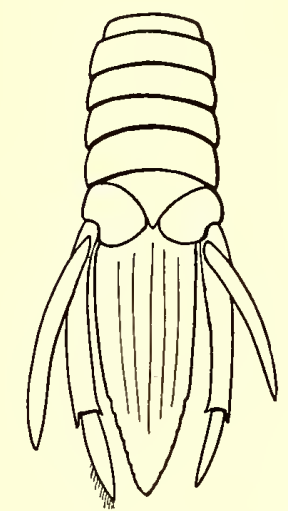

Fig. 11. Katanthura barnardi, Metasoma, $\pm 12 \times$.

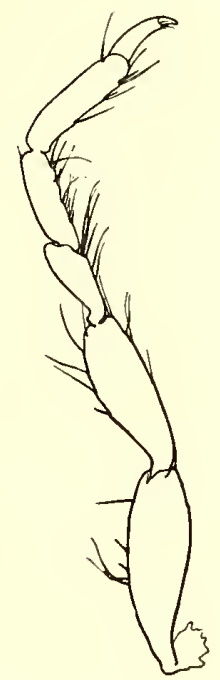

Fig. 12. Katanthura barnardi, Thorakopod VII (Pereiopod IV), $23 \times$.

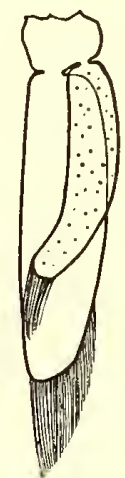

Fig. 13. Katanthura barnardi, Pleopod I, $25 \times$.

selbst. Barnard legt den Nachdruck auf die Tatsache, dass die Form und Oberfläche des Telsons stark variieren kann (8, S. 117 und Taf.). Gewiss ist dies richtig. Bei Katanthura ist es, so weit ich es beurteilen kann, leicht convex, mit 6 der Länge nach verlaufenden sehr schwachen Rücken, sodass der Durchschnitt, welchen ich nicht untersuchen konnte, leicht wellenförmig sein muss. Diese Skulptur ist noch nicht bekannt, einige Übereinstimmung finde ich bei Haliophasma tricarinatum Barnard und Exanthuta filiformis (Lucas) (8, Taf. 4, Fig. 3, 22). Statocysten fehlen durchaus. Die Uropoden (Fig. 11) zeigen nichts besonders.

Mit welchen anderen Formen nun ist Katanthura verwandt? Sie gehört gewiss zu NormaN und Stebbing's Section B (138, S. 122), welcher BARnARD (8, S. 129) übernommen hat, und zwar bei der Abteilung II A 2, zu welcher Paranthura und Cruregens gehören. Das Maxilliped ist aber 4-gliedrig im Sinne BARNARD's, während er bei Paranthura 3- und bei Cruregens 2-gliedrig ist (siehe weiter S. 25).

\section{Metanthura nov. gen.}

Mundteile stechend und saugend. Maxilliped 4-gliedrig. Das fünfte Glied der Thorakopoden V-VIII nicht „underriding” das sechste. 6 Freie Pleomere. Exopod der Uropoden gut entwickelt. Statocysten fehlen.

Metanthura indica nov. spec. (Fig. 15-24)

Stat. $310.8^{\circ} 30^{\prime}$ S. $119^{\circ} 7^{\prime}$ 5. O. 73 m. 1 Exemplar, ㅇ.

Die Länge beträgt $26 \mathrm{~mm}$ (Fig. 14-16). Habitus stabförmig, Farbe gelbweiss mit braunem Fleck auf dem Metasoma. Oostegite fehlen. Augen vorhanden. 
Antennulae (Fig. 17) gut entwickelt, Flagellum mit etwa 30 Gliedern. Antennae länger, Flagellum mit etwa 44 Gliedern.

Mandibula (rechte Fig. 18). Ebenso wie bei Katanthura ist die Mandibula ein konisches, hohles Organ; Uer Rand wird von einem Kranz von Stacheln umgeben. Aus der Öffnung treten wenigstens 3 (vielleicht 4) Lanzette nach aussen; diese Lanzette setzen sich fort in der Mandibula selbst; sie sind

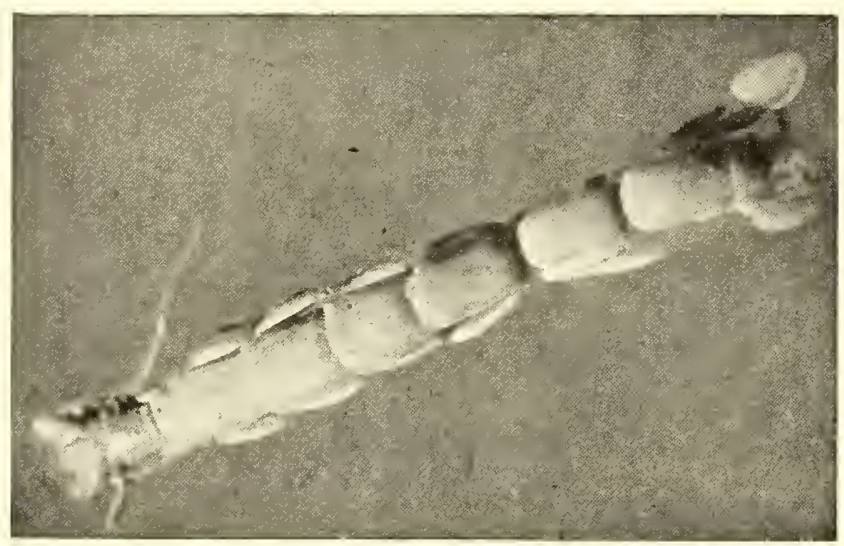

Fig, 15. Metantbura indica 우, $\pm 3 \times$

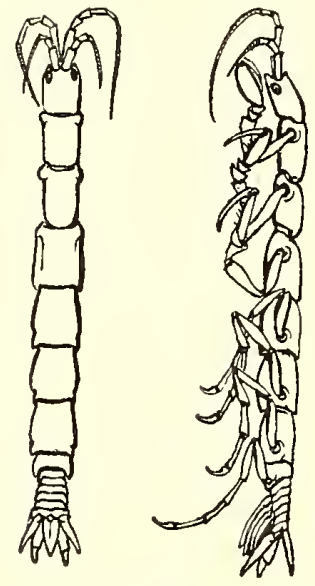

Fig, 14. Metanthura indica 오, $\pm 2 \times$.

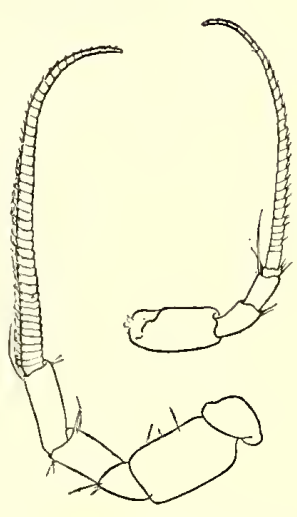

Fig. 17. Metanthura indi. ca. Antennula (rechts) und Antenna (links), $12 \times$.

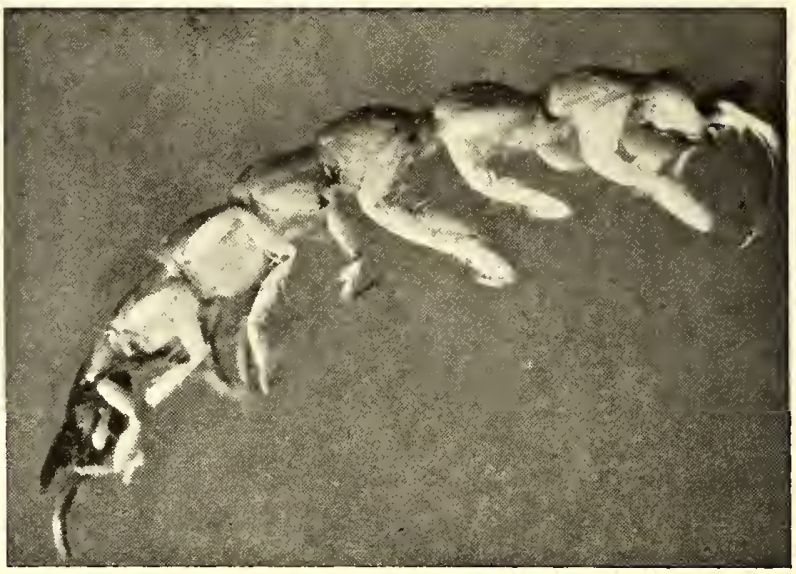

Fig. 16. Metantibura indica $9, \pm 31 / 2 \times$.

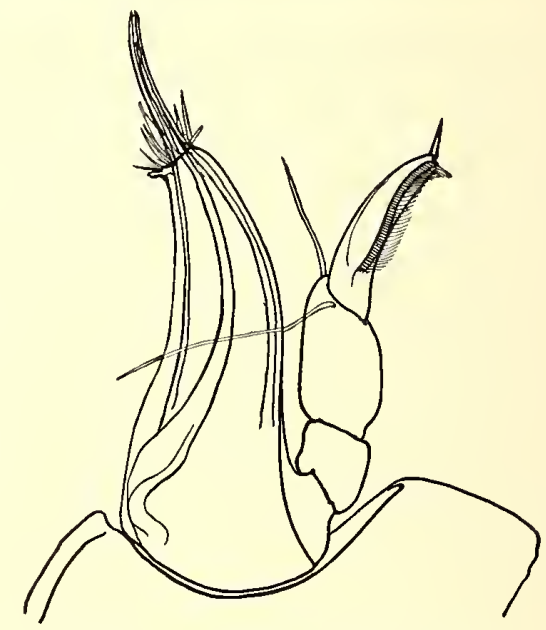

Fig. 18. Metantbura indica, rechts Mandibula, $50 \times$.

Stechorgane und können wahrscheinlich zuriickgezogen werden, sodass die Mandibula als Saugorgan auftreten kann. Meine Zeichnung ist nicht im Widerspruch mit ScHIöDTE's Figur I, welche den Eindruck macht, dass auch bei Calathura brachiata (Stimpson) die Mandibula selbst hohl ist und éin Lanzett heraustreten lässt, statt 3 bei Metanthura. Der Palp der Mandibula ist 3-gliedrig; das dritte Glied trägt an der konkaven Seite einen Kamm von Haaren und eine starke Endstachel.

Mayillula. Die Beschreibung der Maxillula Norman und StebBing's, nur angefüllt durch eine Abbildung von Calatbura brachiata (138, Taf. 26, Fig. $1 \mathrm{mxl}$.), passt sicher nicht für unsre neue Art.

Der Bau der Maxillula ist ganz anders. Fig. 21 gibt an, was sicher zu sehen war. Die Maxillula ist sehr lang und schmal, und hohl. Aus der Öffnung sticht eine Nadel, welche an der konkaven Seite gesägt ist; an ihrer Basis befinden sich Muskelfasern in der Maxillula selbst. Ebenso sieht man in der Maxillula ein Reserve-Stylett vom selben Bau wie der nach aussen ragende. Der ganze Bau 
passt aber vorzüglich bei einer stechenden und saugenden Lebensweise. Auch hier sind Nachuntersuchungen an einem reichen Artenmaterial erwünscht. Maxilliped (Fig. 20; erstes Glied und Epipod nicht gezeichnet) 4-gliedrig; das zweite Glied länger als das dritte und vierte zusammen, welche zahlreiche sehr lange Haare tragen.

Thorakomeren II-VII caudad etwas an Breite zunehmend und sich verkürzend, rechteckig. Thorakomer VIII viel kürzer als die übrigen, aber doch mit Extremitäten. Alle Thorakomeren leicht convex, glatt.

Thorakopod II (Gnathopod I, Fig. 19) starker Greiffuss; III ebenso Greiffuss, aber viel weniger stark; IV ebenso aber mit noch schwächerer Hand. V--VIII (die Pereiopoden) lange, schlanke Extremitäten (Fig. 22), caudad an Länge zunehmend. Bei keinem der Pereiopoden ist der Merus „underriding” im Gegenteil, er ist cylindrisch und nur etwas kürzer als der Carpo-propus; er stimmt ungefähr mit dem von Anthuta überein (p. 114, fig. 2 A).

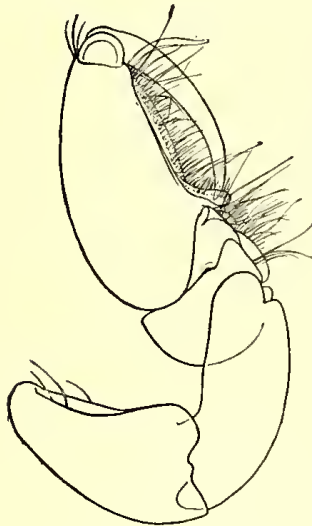

Fig. 19. Metantbura indica, Thorakopod II (Gnathopod I), $12 \times$

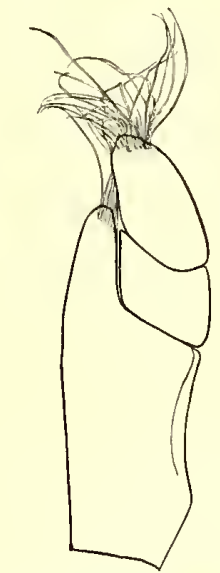

Fig. 20. Methanthura indica, Maxilliped, $33 \times$.
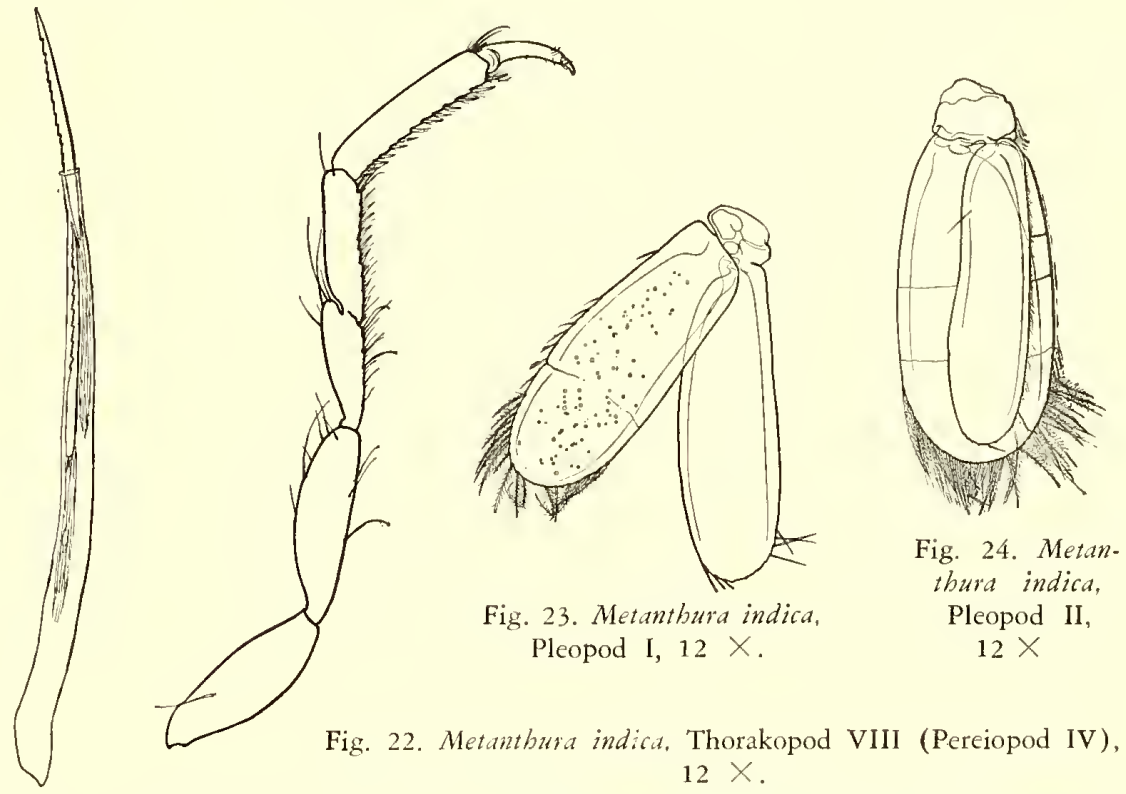

Fig. 21. Metantbura indica, Maxillula, $33 \times$

Pleomeren ungefähr gleich lang. Zwischen VI und dem Telson eine deutliche Naht. Telson glatt, plan-convex, mit schmalem etwas dorsad umgeschlagen Rand.

Pleopode. Das erste Paar (Fig. 23) nicht operculiform; das Exopod mit zahlreichen grauen Flecken und einer Naht. Pleopod II (Fig. 24) mit schmalem Edopod, viel schmäler als das Exopod, welche zwei vollkommene und eine unvollkommene Naht zeigt. Beim dritte Paar ebenso eine unvollkommene Naht. Caudad nehmen die Pleopoden nicht an Grösse ab. Uropoden normal. (Fig. 14).

Metanthura normani nov. spec. (Fig. 25-36)

Stat. 99. $6^{\circ} 7^{\prime} 5^{\prime \prime}$ N. $120^{\circ} 26^{\prime}$ O., 16-23 m. 1 Exemplar $\sigma^{\prime}$.

Die Länge beträgt $13 \mathrm{~mm}$ (Fig. 25, 28, 29). Farbe gelb. Vorderrand mit 2 starken Einbuchtungen. Cephalon viereckig. Augen sehr gross.

Antennulae (Fig. 26) sehr gross und stark. Pedunculus 5-gliedrig, das 4te Glied am kürzesten. Flagellum mit 19 Gliedern, welche, mit Ausnahme der distalen, breit und stark behaart sind.

Antennae (Fig. 30) Pedunculus mit 5 Gliedern, von welchen das dritte am kürzesten. Flagellum mit 25 Gliedern. 


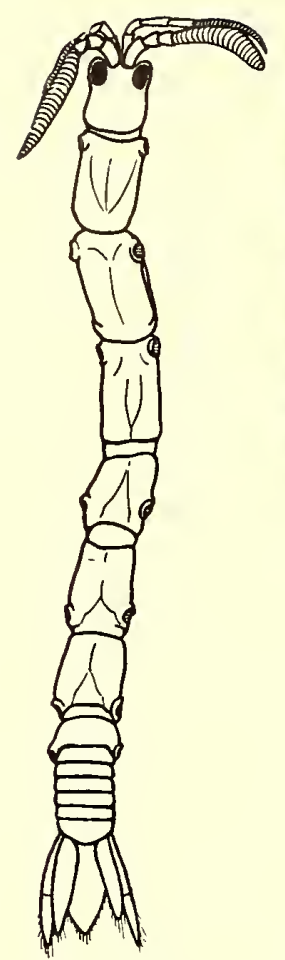

Fig. 25. Metantbura normani $\hat{o}, \pm 17 \times$.

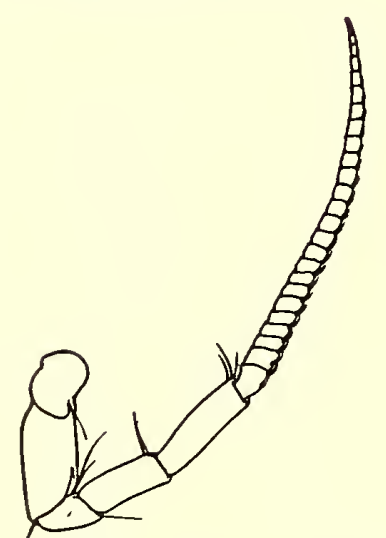

Fig. 30. Metanthura nor. mani, Antenna, $25 \times$.

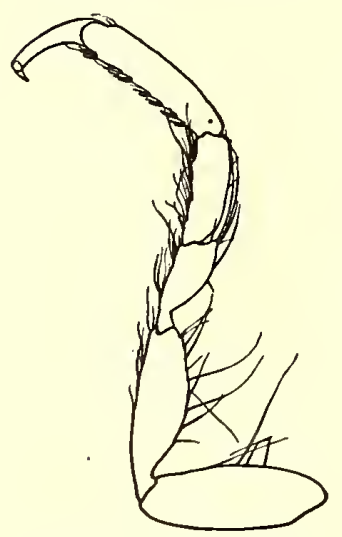

Fig. 33. Metanthura normani, Thorakopod $\mathrm{V}$ (Pereiopod I), $25 \times$.

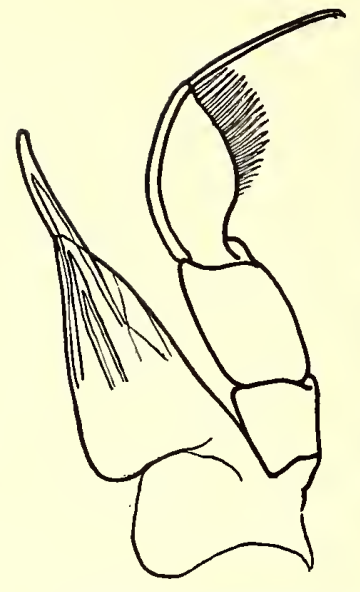

Fig. 27. Metantbura normani, Mandibula, Antennula, $25 \times$.

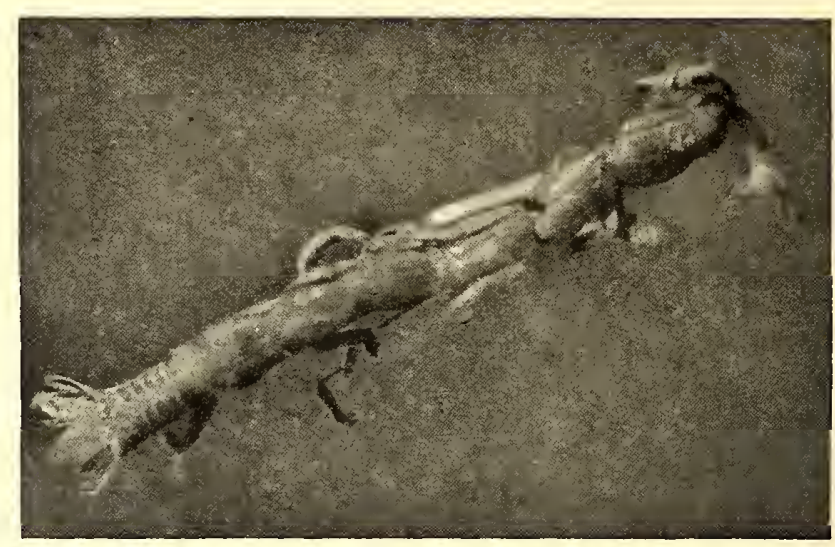

Fig. 28. Metanthura normani $\hat{\delta}, \pm 6 \times$.

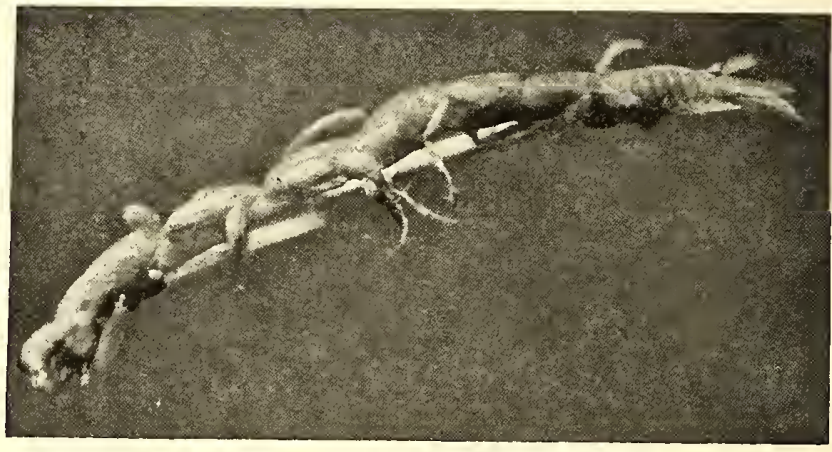

Fig. 29. Metanthura normani $\hat{\delta}, \pm 6 \times$.

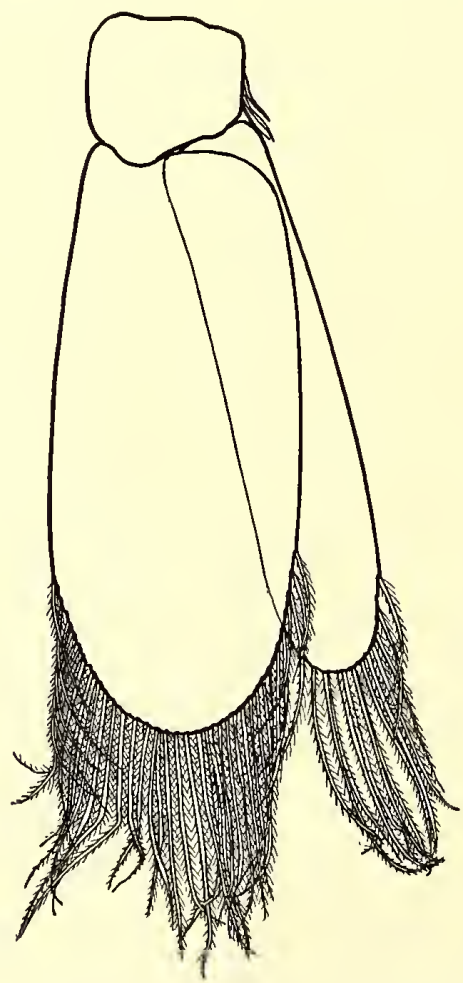

Fig. 35. Metanthura normani, Pleopod I, $25 \times$

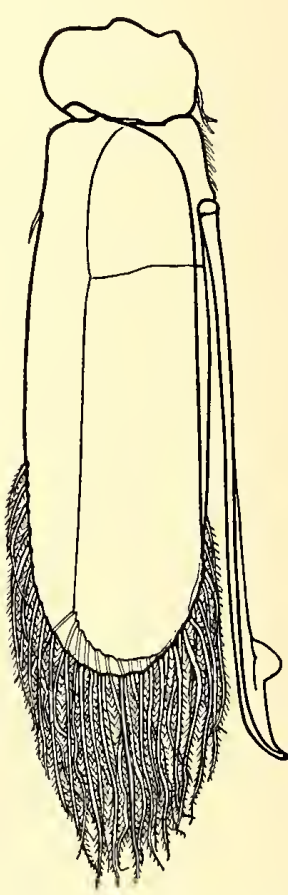

Fig. 36. Metantbura normani, Pleopod II, $24 \times$. 
Die Antennulae und Antennae erstrecken sich bis an dem Hinterrand des zweiten Thorakomers.

Mandibulae (Fig. 27) : beiderseits von gleichem Bau. Die Mandibula ist auch hier hohl, aus der terminalen Öffnung ragt eine Stachel heraus, während distal von ihr sich eine Reserve-Stachel bildet. Endglied des dreigliedrigen Palpus medial stark behaart und mit langer Endstachel.

Maxillula. (Fig. 31): sehr lang und nadelförmig, mit braungefärbter Spitze, welche gezähnelt ist; wahrscheinlich ragt auch hier eine selbständige Stachel heraus, aber Sicherheit habe ich nicht erlangen können; ebenso bleibt es fraglich, ob Reserve-Stacheln vorhanden snd, weshalb ich diese in die Figur nicht aufgenommen habe.

Maxillepeden (Fig. 32): 4-gliedrig (basales Glied nicht gezeichnet); zweites Glied lang mit starkem medialen Fortsatz. Drittes Glied mit 4-5 fadenförmigen, mediad gerichteten Anhängen.

Thorakomeren viereckig, II, III und IV ungefähr gleich lang, V, VI und VII etwas kürzer, VIII stark verkürzt. Die Gnathopoden inserieren nahe am Vorderrand, die Pereiopoden nahe am Hinterrand.

Gnathopod I (Fig. 34): mit starker Greifhand und schwer behaart; die Klaue des Dactylus wahrscheinlich abgebrochen. Caudad nehmen die 3 Gnathopoden an Grösse und Stärke etwas ab.

Pereiopoden (Thorakopoden V-VIII, Fig. 33) : caudad ein wenig an Länge zunehmend. Pleotelson zungenförmig. Dorsale Flache ganz glatt, etwas convex.

Pleopoden (Fig. 35, 36): alle ungefähr gleich gross, Exo- und Endopod ungeteilt. Die Appendix masculina ist länger als die Poden und endet etwas mediad gebogen, spitz, mit einem lamellaren Anhang, welche eine Verbreitung zeigt.

Uropoden: lang und schmal, länger als das Pleotelson.

Ich habe mich abgefragt, ob diese Form vielleicht das Männchen von Metanthura indica representieren könnte. Der Körperbau kommt bei beiden Tieren gut überein; der grosse Unterschied im Bau der Antennula zwischen beiden könnte dann auf eine sexuelle Modification beim Männchen zurückgebracht werden, wie BARNARD diese Möglichkeit im allgemeinen beschreibt (8, S. 111).

Aber der Bau der Mandibula, des Pleons u.s.w. weisen m.E. daraufhin, dass wir es mit einer anderen Art zu tun haben.

Wenn wir jetzt versuchen Katanthura und Metanthura in den Schlüssel BARNARD's (8, S. 129) aufzunehmen, so wird man diesen in folgender Weise erganzen müssen:

I Mit Statocysten u.s.w.

Section B.

\section{Ohne Statocysten}

A. Exopod der Uropoden nicht reduziert

1. Merus der Thorakopoden V-VIII (Pereiopoden I-IV) ,underriding” den Carpo-propus. Maxillipeden 5-gliedrig, Calatbura Norman \& Stebbing.

2. Merus der Thorakopoden V--VIII (Pereiopoden I-IV) nicht ,underriding" den Carpopropus.
a. Maxilliped 4-gliedrig
b. Maxilliped 3-gliedrig
c. Maxilliped 2-gliedrig

B. Exopod der Uropoden reduziert
Katanthura nov. gen.

Metanthura nov. gen.

Paranthura Bate and Westwood

Cruregens Chilton

Pseudantbura Richardson 


\section{Paranthura Bate et Westwood}

1 Paranthura lifuensis Stebbing.

Chinesisches Meer, 72-144 m (8, S. 154); Ochotskisches Meer (77, S. 259; Sandal Bay, Lifu, Loyalty-Inseln (183, S. 625).

2 Paranthura punctata (Stimpson).

Port Adelaide, Golf von St. Vincent; New-Süd-Wales; Tasmanien; New-Seeland (8, S. 154); Waikare Lake, Neu Seeland, 2 m (40, S. 705); Simonis Bay, Kap der Guten Hoffnung (190, S. 420); Umhlangakulu River, 90 m; Kap Morgan, 139 m; Cove Rock, 144 m; Kap Hangklip, 131 m; alle Süd-Afrika (6, S. 349a; 7, S. 343).

3 Paranthura flagellata (Chilton).

Wata mooli; Neu-Süd-Wales, 97-106 m (223, S. 220); Lyttelton; Neu-Seeland (28, S. 172).

4 Paranthura involuta Whitelegge.

Botany Bay, 90—94 m (223, S. 224).

5 Parantbura japonica Richardson.

Mororan, Japan (155, S. 78); Japanisches Meer (78, S. 129); Ochotskisches Meer, Tiefe $3 \mathrm{~m}$ (73, S. $1234 ; 78$, S. $128 ; 77$, S. 259$)$.

6 Paranthura porteri (Borne).

Chili $(8$, S. 155).

7 Paranthura neglecta Beddard.

Kerguelen, $229 \mathrm{~m}$ (14, S. 145).

Die übrigen vier Arten sind atlantisch und mediterran.

\section{Cruregens Chilton}

1 Cruregens fontanus Chilton.

Eyreton, North Canterbury; Leeston; Winchester, South Canterbury; alle Neu-Seeland, unterirdisch in Süsswasser (37, S. 211).

Die einzig bekannte Art.

\section{Pseudanthura Richardson}

1 Pseudantbura lateralis Richardson.

Cape Point, 1620-1800 m (7, S. 344); Küste Sudan's zwischen Dakar und la Praya, 930-3200 m (159, S. 525).

Die einzig bekannte Art.

\section{Colanthura Richardson}

Die einzige Art der Gattung Colanthura Richardson, Colanthura tenuis wird bei den Bermuda Inseln gefunden (149, S. 79). 


\section{Edanthura Boone}

1 Edanthura linearis Boone.

Kalifornien (22, S. 148).

Die einzig bekannte Art.

Weil mir Boone's Arbeit unbekannt ist, kann ich diese Gattung nicht in den Schlüssel BARNARD's (8, S. 128), welchem ich gefolgt habe, einfügen.

Von den Anthuriden ist deshalb bis jetzt noch kein Repräsentant im Indo-Australischen Archipel gefunden worden, überdies kennt man keine Formen aus den Philippinen und dem nördlichen Teil des Pazifischen Ozeans (mit Ausnahme der ungenügend beschriebenen Art Paranthura japonica Richardson).

\section{Unterordnung VALVIFERA}

Familie ARCTURIDAE

\section{Arcturus Latreille}

Die von zUR StRassen vorgeschlagene Einteilung der Gattung Arcturus in Arcturus s. str. und Antarcturus (226, S. 686) hat bei allen Forschern Beifall gefunden und vor einigen Jahren ist diese Geschichte von Nordenstam ausführlich noch einmal beleuchtet worden (136, S. 122). Die Unterschiede zwischen beiden Gattungen liegen jetzt wohl fest; nur in einer Hinsicht braucht unsre Kenntnis noch Erweiterung und zwar von der Marsupialplatten. ZUR STRASSEN hat sich hierüber nicht geäussert; erst später hat STEBвING mit Recht daraufhin gewiesen (189, S. 51) und behauptet, es kämen bei Antarcturus beiderseits 4 solcher Platten vor (189, S. 52); bei Arcturus aber soll diese Zahl nur 3 betragen. Dagegen spricht aber die Meinung Miss Richardson's, welche mit Nachdruck behauptet, dass das Marsupium aus 4 Platten beiderseits bestehen soll (150, S. 99), statt aus 3, wie sie früher angegeben hatte (149, S. 327). Freilich bedürft die Kenntnis der Marsupialplatten der Arcturiden noch weiterer Forschungen, auf welche Tatsache Nordenstair ebenso gewiesen hat (136, S. 118).

Die Gattung Arcturus umfasst jetzt, nach Abtrennung von Antarcturus, etwa 18 Arten, von welchen 15 im Indopazifischen Gebiet vertreten sind; ausserhalb diesem Gebiet fallen $A$. baffini (Sabine), scabrosus Norman und pawneeanus Boone. Es gibt aber einige Arten, welche weit über den $50^{\circ} \mathrm{N}$. Breitengrad südwärts hinaustreten; so parvus Richardson und birsutus Richardson (siehe unten), weiter pawneeanus Boone aus dem Karibischen Meer (23, S. 139). Aus der Beschreibung von parvus und birsutus erhellt sich nicht, ob wir es wirklich mit Arten von Arcturus zu tun haben, aber von Miss Richardson, welche zur Strassen's Arbeit kannte (149, S. 717), ist nicht zu erwarten, dass sie ohne Weiteres nur mit Arcturus Rechnung gehalten hat. Vorläufig bleibt aber nichts anders übrig als die Grenze für Arcturus viel südlicher, bis weit unter dem Aequator zu ziehen, als ZUR STRASSEN getan hat.

1 Arcturus parvus Richardson.

Pitt Passage, Insel Gomono, 2272 m (158, S. 32). 
2 Arcturus birsutus Richardson.

Golf von Tomini. Insel Dodepo, $1165 \mathrm{~m}$; nord-pazifisch, Aleuten und Bering-Meer, 243-19832m (158, S. 32;147, S. 43; 155e S. 97).

Nach Gurjanova (74, S. 1235) gehört diese Art zu den abyssalen Formen des Stillen Ozeans, die nur in dem Bering-, Ochotskischen und Gelben Meer durchdringen, in den Gewässern Japans aber fehlen (78, S. 196).

3 Arcturus hastiger Richardson.

Japan, $43^{\circ} \mathrm{N}, 140^{\circ} 10^{\prime} 30^{\prime \prime} \mathrm{O}$ und $43^{\circ} 01^{\prime} 35^{\prime \prime} \mathrm{N} .140^{\circ} 10^{\prime} 40^{\prime \prime} \mathrm{O} .702-770 \mathrm{~m}(150, \mathrm{~S} .101)$, Ochotskisches Meer (77, S. 259). Japanisches Meer, Tiefe 58-770 m (74, S. 1230, 1233; 78, S. 180).

4 Arcturus granulatus Richardson.

Japan, $46^{\circ} 42^{\prime}$ N. $151^{\circ} 45^{\prime}$ O. und $46^{\circ} 42^{\prime}$ N. $151^{\circ} 47^{\prime}$ O., $412 \mathrm{~m}$ (155, S. 99). Ochotskisches Meer $(78$, S. $190 ; 79$, S. 259).

5 Arcturus crassispinis RichaRdSON.

Sachalin, Ochotskisches Meer, Tiefe 27-32 m (78, S. 188), Japan an vielen Stellen, 38-131 m (150, S. 106); Japanisches Meer, Tiefe 30—150 m (74, S. 1230, 1233; 78, S. 188).

6 Arcturus glaber Benedict.

Aleuten, Pribiloff-Inseln; Alaska Bering-Meer, 65-103 m (16, S. 46; 150, S. 101; 78, S. 194).

7 Arcturus beringanus Benedict.

Alaska, Bering-Meer, Tiefe 55-140 m (149, S. 328); Ochotskisches Meer, Tiefe 22 m; Japanisches Meer, Tiefe 50-80 m (77, S. 258; 78, S. 190).

8 Arcturus baffini (Sabine).

Ochotskisches Meer (77, S. 259; 78, S. 177), Nord-Atlantisch und arktisch, 9-1116 m (88, S. 193, 74, S. 1230) Grönland, 25-250 m (136).

Die Varietät tuberosas G. O. SARs, Davisstrasse, Hudson Bai, Grinnelbucht, Grönland (72, S. 448); Stephensen (198, S. 13; 200, S. 17) fasst typische Art und Varietät zusammen und gibt an, wie diese Art von arktischem Amerika, rund um Grönland, bei Island und zwischen Norwegen und die Farör, wie auch westlich von Spitzbergen gefunden wird bis zu $1100 \mathrm{~m}$ Tiefe, Confer auch SAEMUNDSEN (168, S. 29).

9 Arcturus diversispinis Richardson.

Zwischen Attu, Aleuten und den Kommandor Inseln (150, S. 104), Bering-Meer, Tiefe 270 m, (78, S. $185 ; 77$, S. 260).

10 Arcturus tritaeniatus Richardson.

Bering-Meer $52^{\circ} 12^{\prime} \mathrm{N}, 179^{\circ} 52^{\prime}$ O., $77 \mathrm{~m}(150$, S. 103; 78, S. 194).

11 Arcturus magnispinis Richardson.

Bering-Meer $52^{\circ} 12^{\prime}$ N., $179^{\circ} 52^{\prime}$ O., 77-94 m (150, S. 104, 78, S. 186) Tiefe 80-100 m. Nach Gurjanova $(74$, S. 1230, 1235) autochthon für die asiatische Seite des Stillen Ozeans.

12 Arcturus longispinis Benedict.

Aleuten $52^{\circ} 0.5^{\prime}$ N., $177^{\circ} 40^{\prime}$ W., 99 m (149, S. 329); Bering-Meer, Tiefe $110 \mathrm{~m}$ (78, S. 181, 77, S. 260). 
13 Arcturus brevispinis Richardson.

Zwischen Insel Attu, Aleuten und den Kommandor-Inseln, 243 m (150, S. 105); Bering-Meer (78, S. 189; 77, S. 260). Nach GuRJanova (74, S. 1230, 1235) eine autochthone Form der asiatischen Küsten des Stillen Ozeans.

14 Arcturus ulbani Gurjanova.

N. W. Pazifischer Ozean (72, S. 87, 102); Ochotskisches Meer, Tiefe 8-141 m (77, S. 259; 78, S. 178).

15 Arcturus setosus Gurjanova.

N. W. Pazifischer Ozean (72 m, S. 88, 102) Japanisches Meer, Ochotskisches Meer, Tiere 4-500 m (77, S. 182; 78, S. 258); Im Japanischen und Ochitskischen Meer die beiden Varietäten seminudus, Tiefe bis $30 \mathrm{~m}$, und acuticaudatus, Tiefe 65-124 m (72, S. 89, 90, 103; 74, S. 1233; 78, S. 183).

16 Arcturus crenulatus Gurjanova.

N. W. Pazifischer Ozean (72, S. 90, 103), Japanisches Meer, Tiefe 60 m (78, S. 193; 78, S. 260).

17 Arcturus beddardi Gurjanova.

Ochotskisches Meer (77, S. 259; 78, S. 197), Tiefe 592-1076 m, Pazifisch, typische Form der abyssalen Tiefen des Pazifiks (74, S. 1231).

Bei seiner Analyse der Isopodenfaunen des Beringmeeres, des Ochotskischen Meeres und deз Japanischen Meeres, weist Gurjanova (78, S. 237-250) darauf hin, das von den Formen, die in dem Bering-Meer vorkommen n.l. A. longispinis, $A$. diversispinis, $A$. magnispinis, $A$. brevispinis, $A$. glaber, $A$. tritaeniatus und $A$, birsutus, die ersten $6 \mathrm{zu}$ den autochthonen Flachwasserformen des Beringsees gehören, während $A$. birsutus eine Tiefseeform ist, welche zum Abyssal des Stillen Ozeans gehört. Es besteht hier ein Austausch zwischen der Fauna des Bering-Meeres und dem Eismeer. Arcturus baffini, die gleichfalls in dem Ochotskischen Meer vertreten ist, geht zusammen mit Pleuroprion intermedium (Confer S. 258) nach Osten in die Beaufort-See, weiter längs der Nordküste von Alaska und Kanada und erscheint an den Küsten von Labrador, Baffinsland, West Grönland, Island und den Farör. A. beringanus ist über alle drei vo GuRJANOva genannten Meeren verbreitet.

Als autochthon, endemisch für das Ochotskische Meer verzeichnet GuRJanova Arcturus ulbani, A. granulatus, $A$. setosus, die in diesem Meere sehr gemein ist, $A$. setosus seminudus, $A$. setosus acuticaudalis, letztere jedenfalls sehr allgemein im Ochotskischen Meer und $A$. beddardi. Das Vorkommen von Unterarten in diesem Meere weist daraufhin, dass die Fauna des Ochotskischen Meeres relativ jung ist. Mit dem Japanischen Meer gemein hat das Ochotskisches Meer A. setosus seminudus, A. setosus acuticaudalis, $A$. beringanus. A. hastiger und $A$. crassispinis, die im Japanischen Meer weit verbreitet ist. In den Flachenwasser des Ochotskischen Meeres kommt $A$. bastiger viel vor. Man findet sie auch bei der Aniva Bai. Sie ist in dem nördlichen Teil des Japanischen Meeres durch die La Perouse Strasse eingedrungen.

Als arktische Art des Ochotskischen Meeres soll man $A$. baffini rechnen eine ursprünglich aus der Stillen Ozean stammenden Art, die erst später in die Beaufort See eingedrungen ist.

$\mathrm{Zu}$ den Tiefseeformen gehört $A$. beddardi. Kommt im südlichen Teil des Ochotskischen Meeres vor und ist nach GURJANOva hier wahrscheinlich durch die tiefen Strassen zwischen den südlichsten der Kurillen und dem Stillen Ozean eingedrungen.

Im Japan-Meer findet man als subarktisch-pazifische Arten $A$. beringanus, A. hastiger und Ai. crassispinis, von denen beide letztgenannten Arten auch im nördlichen Teil der Tartarensund vorkommen. Verschiedene Arcturus-Arten sind pazifische autochthone Formen, die sich erst später über die Arktis verbreitet haben. 
1 Astacilla dilatata Richardson.

Japanisches Meer, $38^{\circ} 16^{\prime}$ N., $138^{\circ}$ 52' O., 126 m (155, S. 97 confer auch 78, S. 204; 78, S. 245). Nach GuR JANOVA ist $A$, dilatata eine pazifisch-boreale Form.

2 Astacilla polita Gurjanova.

Japanisches Meer bei der Petrowinsel, 60 m (77, S. 256; 78, S. 205) nach GuRJanova (78, S. 245) eine endemische Form des Japanischen Meeres.

3 Astacilla californica Boone.

Venice, California (20, S. 601).

4 Astacilla kerguelensis Vanhöffen.

Kerguelen (218, S. 523).

5 Astacilla marionensis Beddard.

Marion Insel, 180 m., Kerguelen, Oberfläche (13, S. 108).

6 Astacilla mediterranea Koehler.

Imkomaas river, Süd-Afrika 72 m (7, S. 389); Villefranche, Mittelmeer (102, S. 44).

7 Astacilla bacillus Barnard.

Walker Point bei Knysna, 85 m; O'Neil Peak, Zululand, 99 m (7, S. 387).

8 Astacilla diomedea Benedict.

Strasse von Magellan, $31 \mathrm{~m}$ (16, S. 51).

Die Gattung umfasst 20 Arten, von denen also höchstens 8 im Indopazifischen Gebiet vorkommen. Über die Verwandschaft dieser Formen kann ich leider nichts anführen; amblyura scheint mit granulata (G. O. Sars) vom Nord-Atlantischen Ozean, mit marionensis und mit longicornis (G. O. Sars) vom Nord-Atlantischen Ozean übereinzustimmen. Ganz überwiegend aber ist die Gattung atlantisch. - A. bocagei Nobre ist mir unbekannt.

\section{Neastacilla 'Tattersall}

1 Neastacilla amblyura (Stebbing)

Golf von Manaar (188, S. 148).

2 Neastacilla deducta Hale.

Port Adelaide, Süd-Australien (82, S. 34), Port River, Süd-Australien (84, S. 313).

3 Neastacilla algensis Hale.

Golf von St. Vincent, 9-11 m (81, S. 212; 83, S. 313).

4 Neastacilla falclandica (Ohlin).

North Cape, Neu-Seeland, 126 m (205, S. 244). Falkland-Inseln, 1 m (139, S. 267; 193, S. 353; 136, S. 122).

5 Neastacilla magellenica (Ohlin).

Magellanstrasse, $18 \mathrm{~m}$ (139, S. 268), Falkland Inseln 12-40 m (136, S. 122).

Die beiden von HALE beschriebenen Formen algensis und deducta habe ich bei Neastacilla aufgenommen, obwohl Nordenstam sie offenbar nicht zu dieser Gattung rechnet $(136$, S. 119). 


\section{Idarcturus Barnard}

1 Idarcturus platysoma Barnard.

Atlantische Küste der Kap der Guten Hoffnung und Kapstadt (6, S. 432).

Die einzig bekannte Art.

\section{Neoarcturus Barnard}

1 Neoarcturus oudops Barnard.

Cape Point, Süd-Afrika, 1260-1359 m (4, S. 216; 7, S. 398).

Die einzig bekannte Art.

Die verwandte Gattung Arcturopsis Koehler ist atlantisch.

\section{Arcturina Koehler}

1 Arcturina hexagonalis Barnard.

Kap St. Blaize, Süd-Afrika, 76 m (9, S. 403).

Die zweite bekannte Art, rhomboidalis Koehler, ist atlantisch (Kap Verde).

\section{Pseudarcturella Tattersall}

1 Pseudarcturella chiltoni Tattersall.

Spirits Bay bei North Cape, Neu-Seeland, 3 m (205, S. 245).

Die einzig bekannte Art.

\section{Arcturella G. O. Sars}

\section{Arcturella cornigera (Stebbing)}

Port Elizabeth, Algoa-Bai (4, S. 207); Falscher Bai; Simons Town (218, S. 525); Walker Point bei Knysna; Sebastian Bluff W.; Robben-Insel; alle Süd-Afrika, 43-85 m (7, S. 392); Saldanha-Bai (205, S. 889).

Die Varietät sulglabra Barnard: St. Helena Bai, Mosselbai, Süd-Afrika; 36-144 m (4, S. 211; 9, S. 404).

2 Arcturella lineata (Stebbing).

Port Elizabeth (4, S. 207); Falscher Bai; Agulhas-Bank; Algoa Bai; alle Süd-Afrika; 36-180 m (7, S. 393).

3 Arcturella pustulata Barnard.

Umkomaas-Fluss; Durnford Point; Küste Natals; alle Süd-Afrika; 11-72 m (7, S. 395).

4 Arcturella longipes Barnard.

Tafelbai; Kap St. Francis, Süd-Afrika, 40-135 m (7, S. 396).

5 Arcturella brevipes a Brnard.

Agulhas-Bank(?); Falscher Bai, 40 m (7, S. 397). 
6 Arcturella lobulata Barnard.

Natal, 72 m (9, S. 404).

Die übrigen 7 Arten der Gattung sind atlantisch.

\section{Parastacilla Hale}

1 Parastacilla truculenta Hale.

Beachport, Süd-Australien, 5-7 m (81, S. 210; 84, S. 311 ).

2 Parastacilla bakeri Hale.

Marine Reef, Süd-Australien (81, S. 211; 84, S. 312).

Keine anderen Arten sind bekannt.

\section{Pleuroprion zur Strassen}

1 Pleuroprion chuni (zur Strassen).

Agulhas Bank, 156 m (226, S. 687).

2 Pleuroprion murdochi (Benedict).

Bering-Meer (77, S. 259), Point Franklin, Alaska, 26 m (16, S. 49); Island oder Farör (88, S. 196). Confer auch Gurjanova (74, S. 1232; 78, S. 200).

3 Pleuroprion intermedium (Richardson).

Knyska Harbor, Aleuten, 18 m (149, S. 344; 78, S. 201) Bering-Meer (77, S. 260).

4 Pleuroprion hystrix G. O. Sars.

Ochotskisches Meer, Bering-Meer, amphiboreale Form (74, S. 1231; 77; S. 259, 260; 78, S. 200) Helgoland, Farör, Lofoten und Nord-atlantisch, 630-1044 m (88, S. 195). Es ist diese Form eine amphiboreale Form im Sinne BERG's (18).

5 Pleuroprion tarasovi Gurjanova.

Ochotskisches Meer, Tiefe 122 m, (77, S. 259; 78, S. 202). Autochthone Form des Ochotskischen Meeres.

Die sechste Art Pl. frigidım Hansen ist bei Ost-Grönland, 90-315 m, gefunden worden (88, S. 197) auch von NordenSTAM, bei einer Tiefe von $0-80 \mathrm{~m}(137$, S. 4).

Nach Gurjanova (78, S. 241-244) geht Pleuroprion intermedium durch die Beringstrasse in die Tsuktscha- und Beaufort-See. Das Entstehungszentrum dieser Gattung soll im nördlichen Teile des Stillen Ozeans liegen. Die Gattung dringt in der Arktis nach Osten und Westen vor ohne dabei vollständig cirkumpolär zu werden.

Mehrere Arten von Arcturus (siehe dort) und Pleuroprion sind pazifische Formen, dort autochthon, und haben sich erst später über die Arktis verbreitet.

\section{Antarcturus zur Strassen}

Stebbing (189, S. 53) nennt alle Arten welche s. E. zu Antarcturus gehören. Später hat Nordenstam diese Sache nochmals zur Hand genommen und die Gattung Antarcturus wieder in 
zwei Gattungen geteilt, nl. Antarcturus s. str. und Microarcturus, zu welch letzterer dann 14 Arten gehören sollen (136, S. 128). Von der ursprünglich riesigen Zahl der Arten von Antarcturus s. str. bleiben also noch 34 Arten übrig (Nordenstam hat A. brunneus (Beddard) in seine Liste nicht aufgenommen, obschon er (136, S. 138) wohl eine neue Varietät spinulosus dieser Art beschrieben hat).

Überdies hat NoRdenstam daraufhin gewiesen, dass die Gattung Dolichiscus Richardson mit Antarcturus und Microurcturus verwandt ist, (136, S. 127), sodass diese drei Gattungen zusammen eine ziemlich geschlossene Gruppe bilden dürfen.

\section{Antarcturus cormutus (Beddard).}

Stat. $45.7^{\circ} 24^{\prime}$ S., $118^{\circ} 15^{\prime} 2^{\prime \prime}$ O., $794 \mathrm{~m}, 3$ Exemplare $Q$.

Stat. $85.0^{\circ} 36^{\prime} 5^{\prime \prime}$ S., $119^{\circ} 29^{\prime} 5^{\prime \prime}$ O., $724 \mathrm{~m}, 1$ Exemplar $Q$.

Stat. 88. $0^{\circ} 34^{\prime} 6^{\prime \prime}$ N., $119^{\circ} 8^{\prime} 5^{\prime \prime}$ O., $1301 \mathrm{~m}, 1$ Exemplar $q$.

Stat. $122.1^{\circ} 58^{\prime} 5^{\prime \prime}$ N., $125^{\circ} 0^{\prime} 5^{\prime \prime}$ O., 1165-1264 m, 1 Exemplar $\bigcirc$.

Stat. $316.7^{\circ} 19^{\prime} 4^{\prime \prime}$ S., $116^{\circ} 49^{\prime} 5^{\prime \prime}$ O., $538 \mathrm{~m}, 2$ Exemplare o u. $Q$.

Schon aus dem Archipel bekannt: zwischen Djilolo und der Insel Makyan, $536 \mathrm{~m}$; Golf von Tomini, Celebes, Insel Dodepo, 1165 m. (158, S. 31); weiter Samboangan, $4^{\circ} 33^{\prime}$ N., $127^{\circ} 6^{\prime}$ O., $900 \mathrm{~m}(14, \mathrm{~S} .94)$.

\section{Antarcturus myops (Beddard).}

Stat. $2115^{\circ} 40^{\prime} 7^{\prime \prime}$ S., $120^{\circ} 45^{\prime} 5^{\prime \prime}$ O., $1158 \mathrm{~m}, 2$ Exemplare $10^{\prime \prime}$ und $Q$ mit zahlreichen jungen Tieren im Brutraum.

Beide Exemplare sind ein wenig länger als BEDDARD angibt $(10 \mathrm{~mm})$, nl. $15 \mathrm{~mm}$. Ausser einigen kleinen Unterschieden stimmen die Tiere gut überein mit BEDDARD's Beschreibung; nur sind Augen vorhanden, aber das Pigment fehlt durchaus. BEDDARD's Exemplare sind gefunden worden in der Nähe New-Seeland's, 37 34' S., 179*22' O., in einer Tiefe von 1260 m (14, S. 100).

Aus dem Archipel ist diese Form bekannt von der Makassar-Strasse, 720 m. (158, S. 32).

3 Antarcturus multispinis (Benedict).

Stat. $87.0^{\circ} 32^{\prime} \mathrm{S} .119^{\circ} 39^{\prime} 8$ O., $655 \mathrm{~m}, 1$ Exemplar .

Das Tier ist stark verstümmelt, der Kopf fehlt. Soweit sich beurteilen lässt, bestehen keine wichtigen Gründe es nicht zu multispinis zu rechnen.

Weiter bekannt von den Galapagos-Inseln, $0^{\circ} 24^{\prime}$ S. $89^{\circ} \mathrm{W}, 162 \mathrm{~m}(16, \mathrm{~S} .49)$.

\section{Antarcturus abyssicola (Beddard).}

Zwischen Australien und New-Guinea, $12^{\circ} 8^{\prime}$ S. $145^{\circ} 10^{\prime}$ O., $2520 \mathrm{~m}$; Low-Archipelago, $22^{\circ} 21^{\prime}$ S. $150^{\circ} 17^{\prime}$ W. $4293 \mathrm{~m}$ (14, S. 99), Chatham-Insel, $1512 \mathrm{~m}$ (163, S. 159).

\section{Antarcturus spinifrons (Beddard).}

Insel Kandavu, Fidschi-Inseln, $19^{\circ} 7^{\prime}$ 50" S. $178^{\circ} 19^{\prime} 35^{\prime \prime}$ O., 1908 m, (14, S. 95$)$.

6 Antarctnius addreanus (Hodgson).

Süd-Sandwich Inseln (25, S. 401); Kap Adare, 36-47 m (97, S. 249); $78^{\circ} 25^{\prime} 40^{\prime \prime}$ S., $185^{\circ} 39^{\prime}$ 06 " O., $540 \mathrm{~m}(98$, S. 37). 
7 Antarcturtes antarcticus Bouvier.

Süd-Sandwich-Inseln (25, S. 401); Shag Rock Bank, 160 m; Graham Region, 125-150 m (136, S. 153).

8 Antarcturus kladophorixs Stebbing.

Kap St. Blaize, Süd-Afrika, 229 m (190, S. 435); Kap St. Francis, Süd-Afrika, 135 m (4, S. 213).

9 Antarcturus similis Barnard.

Süd-Afrika an verschiedenen Stellen, $72-216$ m (9, S. 398).

10. Antarcturus brunneus (Beddard).

$46^{\circ} 16^{\prime}$ S. $48^{\circ} 27^{\prime}$ O., $2880 \mathrm{~m}$ (14, S. 98).

Die Varietät spinulosus Nordenstam: Shag Rock Bank, $53^{\circ} 34^{\prime}$ S., $43^{\circ} 23^{\prime}$ W., $160 \mathrm{~m}$; Süd-Georgien $54^{\circ} 17^{\prime}$ S., $36^{\circ} 28^{\prime}$ W., $75 \mathrm{~m}$ und $54^{\circ} 11^{\prime}$ S., $36^{\circ} 18^{\prime}$ W., $252-310 \mathrm{~m}(136, \mathrm{~S} .143)$.

11 Antarcturus studeri (Beddard).

Kerguelen, $49^{\circ} 28^{\prime}$ S., $70^{\circ} 30^{\prime}$ O., $40 \mathrm{~m}$ und $48^{\circ} 45^{\prime}$ S., $69^{\circ} 14^{\prime}$ O., $229 \mathrm{~m}$ (14, S. 102).

12 Antarcturus furcatus (Studer).

Kerguelen an verschiedenen Stellen, $10-229 \mathrm{~m}$; Heard-Insel $135 \mathrm{~m} ; 65^{\circ} 42^{\prime} \mathrm{S}$., $79^{\circ} 49^{\prime}$ O., $3015 \mathrm{~m}$ (14, S. 86; 203, S. 14; 218, S. 519); Kap Adare 81-90 m; Inaccessible Island, 400—434 m; Barne Glacier, Mac Murdo Sound, $360 \mathrm{~m} ; 77^{\circ} 46^{\prime}$ S., $166^{\circ} 8^{\prime}$ O., $540 \mathrm{~m}$ (205, S. 238); Shag Rock Bank 160 m; Süd-Georgien, 75-310 m; Graham Region, 125 m (136, p. 134).

Die Varietät polaris (Hodgson): Victoria-Land. Ross-Meer; Graham Region, 81-540 m (136, S. 134).

13 Antarcturus spinosus (Beddard).

Marion-Insel, $46^{\circ} 46^{\prime}$ S., $45^{\circ} 31^{\prime}$ O., $2475 \mathrm{~m}$ (14, S. 90). Trinidad Channel, Patagonien, $54 \mathrm{~m}$ (109, S. 76).

14 Antarcturus coppingeri (Miers).

Trinidad, Channel, Patagonien, 54 m (109, S. 76).

15 Antarcturus americanus (Beddard).

$52^{\circ} 20^{\prime}$ S. $67^{\circ} 39^{\prime}$ W., $99 \mathrm{~m}(14$, S. 105$) ; 42^{\circ} 24^{\prime}$ S., $61^{\circ} 38^{\prime}$ W. und $48^{\circ} 37^{\prime}$ S., $65^{\circ} 46^{\prime}$ W.; Martha's Bank, Magellanstrasse, 77-180 m (139, S. 270); Ost-Patagonien (16, S. 48); FalklandInseln (136, S. 138). Tiefe $40 \mathrm{~m}-180 \mathrm{~m}$.

16 A. granulosus Nordenstam.

$53^{\circ} 34^{\prime}$ S., $43^{\circ} 23^{\prime}$ W., $160 \mathrm{~m}$; $54^{\circ} 11^{\prime}$ S., $36^{\circ} 18^{\prime}$ W., 252-310m (136 S. 157).

Die übrigen Arten: franklini (Hodgson), biemalis Hodgson, bodgsoni Richardson, oryx zur Strassen von Broughton Island, Port Stephensen, $45 \mathrm{~m}$ habe ich nicht aufgenommen, weil die Beschreibung Haswell's (92, S. 194, 195) nicht ausreicht; ebensowenig $A$. tuberculatus (Thomson) von Dunedin und Lyttelton, $7 \rightarrow$ m (208, S. 416; 210, S. 206).

Die übigen Arten: franklini (Hodgson), hiemalis Hodgson, hodgsoni Richardson, oryx zur Strassen, glacialis (Beddard), anna (Beddard), purpureus (Beddard), gaussianıts Vanhöffen, drygalskii Vanhöffen, tenuispinis (Benedict), lillei Tattersall, borridus Tattersall, belgicae Monod, floridamus Richardson und carribaeus Richardson sind rein antarctisch oder atlantisch. 


\section{Microarcturus Nordenstam}

1 M. oculatus (Beddard).

Port Philip, $38^{\circ} 22^{\prime} 30^{\prime \prime}$ S., $144^{\circ} 36^{\prime} 30^{\prime \prime}$ O., 59 m (14, p. 104); Botany und Wata Mooli, 90-106 m (225, S. 408).

$2 M$. dentatus (Whitelegge).

Botany Bai, 90-94 m (225, S. 410).

3 M. alcicornis (Whitelegge).

Botany Bai, 90-94 m (225, S. 412).

4 M. nodosus (Whitelegge).

Botany Bai, 36-50 m (225, S. 414).

5 M. serrulatus (Whitelegge).

Wata Mooli, Neu-Süd-Wales, 97-106 m (225, S. 416).

6 M. simplicissimus (Whitelegge).

Wata Mooli, Neu-Süd-Wales, 90-106 m (225, S. 408).

7. M. similis (Barnard).

Süd-Afrika an verschiedenen Stellen, $72-216$ m (9, S. 398).

8 M. stebbingi (Beddard).

Kap Maclear, Süd-Afrika, 54 m; Kerguelen (14, S. 106); Shag Rock Bank; Süd-Georgien, 75-310 m (136, S. 163).

9 M. rugosus Nordenstam.

Shag Rock Bank, $53^{\circ} 34^{\prime}$ S., $43^{\circ} 23^{\prime}$ W., 160 m; Graham Region, 62 $55^{\prime}$ S., $55^{\circ} 57^{\prime}$ S., $104 \mathrm{~m}(136$, S. 167).

10. $M$. digitatus Nordenstam.

Shag Rock Bank, $53^{\circ} 34^{\prime}$ S., $43^{\circ} 23^{\prime}$ W., $160 \mathrm{~m}$; Süd-Georgien, $54^{\circ} 11^{\prime}$ S., $36^{\circ} 18^{\prime} \mathrm{W} ., 252-310 \mathrm{~m}$ (136, p. 171).

Die übrigen Arten: patagonicus (Ohlin), kophameli (Ohlin), acanthurus (Monod) und birticornis (Monod) sind antarktisch oder atlantisch.

Die Gattung Dolichiscus Richardson mit den beiden Arten D. pfeifferi Richardson und meridionalis (Hodgson) ist antarktisch.

\section{Familie AmEsopodidaE}

Nach Nordenstam (136, S. 113) hält diese Familie zusammen mit der nächsten Familie, die Pseudidotheidae eine Mittelstellung zwischen den Idotbeidae und den Arcturidae.

\section{Amesopous Stebbing}

1 Amesopous richardsonae Stebbing.

Golf von Manaar (188, S. 46).

Die einzig bekannte Art. 


\title{
Familie PsEudidotheIdAE
}

\section{Arcturides Studer}

1 Arcturides cormutus Studer.

$64^{\circ} 51^{\prime} 7^{\prime \prime}$ O., $47^{\circ} 18^{\prime} 15^{\prime \prime}$ S., 207 m. (203, S. 17); Insel Marion, $46^{\circ} 41^{\prime}$ S. $38^{\circ} 10^{\prime}$ O., $558 \mathrm{~m}$ (14. S. 109).

Die zweite Art, A. miersi (Studer) ist gefunden worden an der Ostküste der Südspitze Süd-Amerika's: $47^{\circ} 1^{\prime} 6^{\prime \prime} \mathrm{S}$.

$63^{\circ} 29^{\prime} 6^{\prime \prime}$ W., $113 \mathrm{~m}$ und $43^{\circ} 6^{\prime}$ S. $60^{\circ}$ W. (203, S. 17; 139, S. 281).

\section{Holidothea Barnard}

1 Holidothea unicornis Barnard.

Walker Point bei Knysna; Knysna Heads; Cape Seal; Agulhas Bank; 70—94 m (7, S. 384).

Die einzig bekannte Art.

\section{Pseudidothea Ohlin}

Die Gattung Pseudidotheca Ohlin mit der einzigen Art Ps. bonnieri Ohlin ist atlantisch (Patagonien, Falkland Inseln).

\author{
Familie IDOTHEIDAE \\ Subfamilie Id ot he in a e \\ GRUPPE I VON COLLINGE $(56$, S. 71)

\section{Cleantis Dana}

TAtTtersall gibt (206, S. 425) eine ausführliche Darstellung von den Schwierigkeiten, welche bei der Wahl zwischen Cleantis Dana und Zenobiana Stebbing auftreten. Er selbst zieht es vor beide Gattungen getrennt zu halten; ich halte es aber für besser alle Arten unter Cleantis zusammenzufassen. Denn dass die zwei Gattungen identisch sind, ist doch wohl sehr wahrscheinlich; ein genauer Vergleich der Mundteile fehlt aber bis jetzt. Überdies ist die Sache mit den Uropoden bei Cl. linearis Dana dem Typus der Gattung Cleantis und Cl. strasseni Thielemann nicht in Ordnung, eine Tatsache, auf welche TAT'TERSALl ebenfalls hinweist. Weiter kann das Uropod durch eine schräge Linie in zwei Teile geteilt sein, wie dies gewöhnlich vorkommt; bei Cl. planicauda Benedict fehlt diese aber (149, S. 406). Dabei kommt noch, dass die Zahl der Glieder des Maxillipeds stark wechseln kann (von 3?-5); ebenso die Zahl des Flagellums der Antennae (1--5), während die Zahl der Pleomeren von $1-5$ geht. Ob deshalb bei Cleantis nicht Arten anderer Gattungen eingereiht worden sind, kann. man. ruhig als fraglich betrachten; jetzt lässt diese Sache sich schwer entscheiden und $\mathrm{m}$ E. ist es nur ratsam alle Arten vorläufig unter Cleantis zusammenzufassen.

Zum Teil sind die Mundteile bekannt; von $\mathrm{Cl}$. annandalei Tattersall kennt man die Maxillula. (206, S. 29) und, ebenso wie von vielen anderen Arten, die Maxillipeden. Soweit mir bekannt ist, sind die Mandibulae und Maxiliae noch nicht beschrieben worden. Collinge sagt, dass speziell 
die Maxillulae und Maxillipeda gute Merkmale für die Unterschiede zwischen den Gattungen und Arten der Valviferen liefern sollen (56, S. 68); ob dies aber auch nicht für die Maxillae und Mandibulae der Fall gilt, weiss ich nicht.

\section{Cleantis japonica Richardson (fig. $37-46$ )}

Stat. 140, Bai von Batjan, in Plankton, 1 Exemplar, ô.

Miss Richardson hat diese Art ausführlich beschrieben, und zwar ein Weibchen aus Japan (164, S. 28). Die Unterschiede zwischen unsrem Männchen und diesem Weibchen sind unbedeutenò.

Länge $12 \mathrm{~mm}$. Breite 21/2 mm. Oberfläche glatt, nicht fein granuliert. Die Körperform stimmt genau mit der von Miss RICHARDSON beschriebenen überein; nur war ihr Weibchen viel grösser $(18.5 \mathrm{~mm})$. Die mediane Protuberanz auf dem Metasoma ist aber kaum vorhanden und eine Tuberkel fehlt durchaus (Fig. 38).

Die 4-gliederige Antennula zeigt nichts besonders (Fig. 39); das Endglied ist sehr klein. Bei Miss Richardson's Exemplar soll das Basalglied in der Mitte eine kleine Tuberkel tragen; diese habe ich nicht entdecken können. Auch die Antenna (Fig. 40) ist normal; das Flagellum ist dicht mit

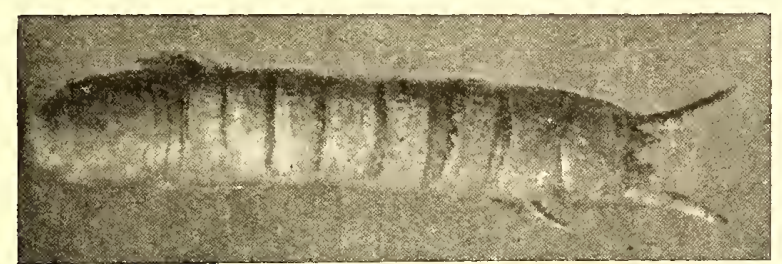

Fig. 37. Cleuntis japonica Richardson, î, $\pm 5 \times$.

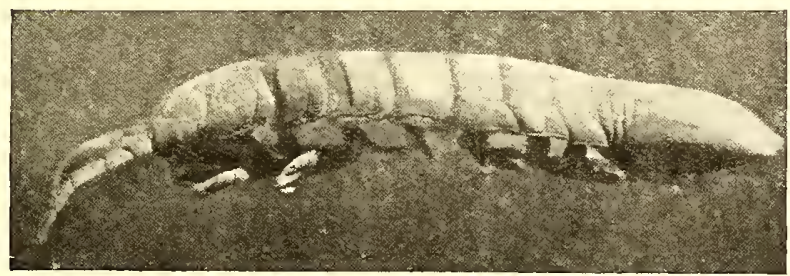

Fig. 38. Cleantis japonica Richardson, $\delta$ von der linken Seite, $\pm 5 \times$

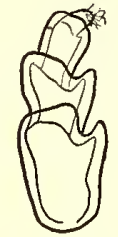

Fig. 39. Cleantis japonica, Antennula, \pm $24 \times$.

Fig. 40. Cleantis japonica, Antenna, $\pm 24 \times$.

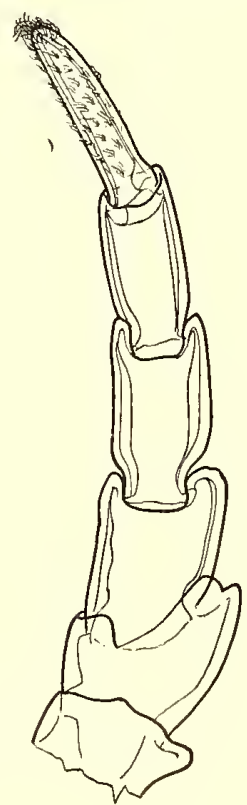

Haarbündeln besetzt. Miss Richardson vermeldet noch ein winziges Endglied; dieses habe ich aber nicht auffinden können; mit gutem Wille konnte ich vielleicht eine sehr dünne, aber jedenfalls unvollkommene Naht nahe dem Ende des Flagellums sehen.

Miss Richardson gibt keine Nachrichten über die Mundteile. Die Mandibulae haben einen sehr komplizierten Bau. Die linke (Fig. 41) zeigt eine Pars incisiva (p.i.) mit etwa 5 starken Zähnen und eine Lacinia mobilis (l.m.) mit starken Bürsten. Zwischen den 5 Zähnen der Pars incisiva und der Lacinia findet man noch 2 Zähne (a); weiter kommen noch mehrere Zähne vor proximal von der Pars incisiva und noch eine Zweite Lacinia ( $\left.\mathrm{lm}^{\prime}\right)$, proximal von der genannten. Der Processus molaris (p.m) besteht wenigstens aus 2 Lappen, vielleicht aus 3. In wieweit alle diese Anhänge an 
einer Seite der Mandibula liegen (die ventrale) lässt sich nicht sagen; sehr wohl möglich ist es, dass ein Teil sich an der dorsalen Seite befindet; die isolierte Mandibula ist zu dünn um dies mit Sicherheit auszumachen; es schimmern im Präparat die verschiedenen Anhänge in verschiedenen Niveaus durch, aber die genauen Verhältnisse bleiben dunkel. Einfacher von Bau ist die rechte Mandibula (Fig. 42),

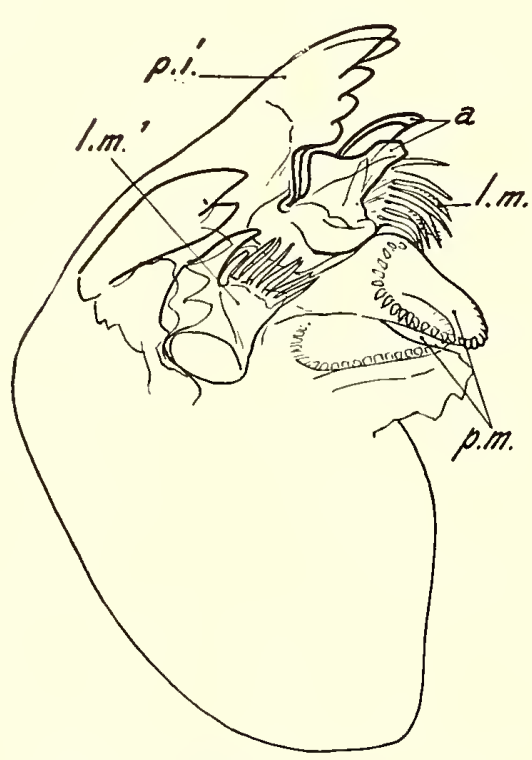

Fig. 41. Cleantis japonica, linke Mandibula, $66 \times$.

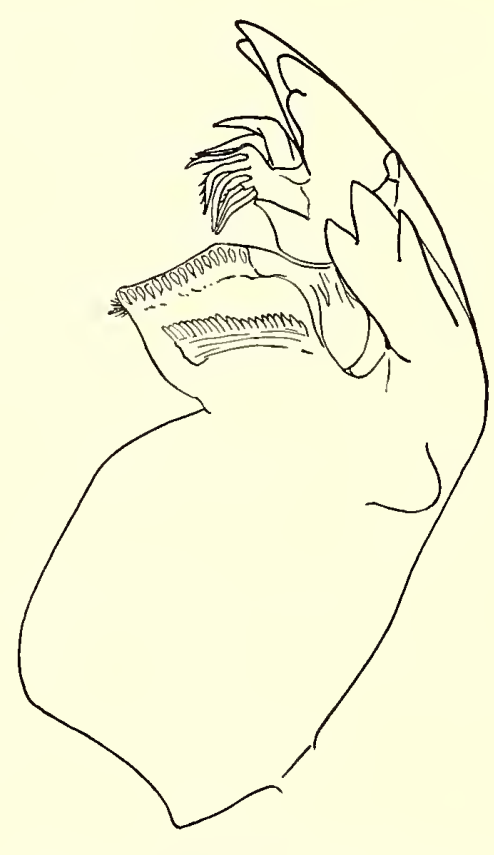

Fig. 42. Cleantis japonica, rechte Mandibula, $66 \times$.

aber doch ist auch diese komplizierter von Bau, als gewöhnlich für die Mandibulae angegeben wird. Der Processus molaris ist einfach und zeigt zwei Kämme.

Die Maxillula (Fig. 43) zeigt diese Eigenartigkeit, dass auf dem Articulus tertius die Reihe der etwa 10 Stacheln sich verdoppelt, was auf dem Articulus primus nicht der Fall ist. Ich verweise nach den Verhältnissen, welche ich bei Idothea
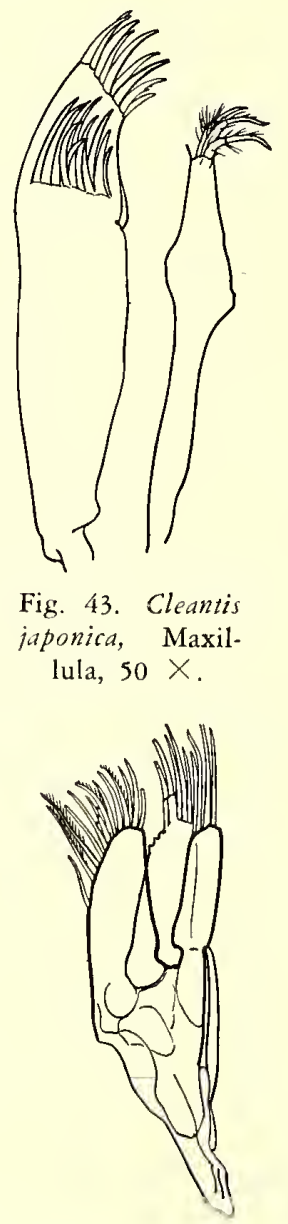

Fig. 44. Cleantis japonica, Maxilla, $50 \times$.

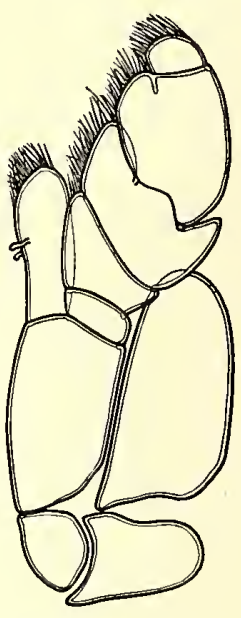

Fig. 45. Cleantis japonica, Maxilltped, $37 \times$.

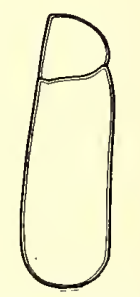

Fig. 46. Cleantis japonica, Uropod, $8 \times$. duplicata gegeben habe (P.). Bei der Maxilla kommen dergleiche Verdoppelungen nicht vor (Fig. 44). Der Maxilliped hat einen 5-gliedrigen Palp (Fig. 45). Die übrigen Thoracopoden stimmen mit den von Miss Richardson's beschriebenen überein. Die Uropoden zeigen 2 Glieder; sie unterscheiden sich von denen von Cleantis strasseni Thielemann und annandalei Tattersall durch das Fehlen des gefiederten Anhanges (207, S. 67; 206, S. 430).

Trotz der genannten Unterschiede finde ich keinen Anlass eine neue Art zu schaffen; höchstens könnte man von einer lokalen Form sprechen.

2 Cl. strasseni Thieleman.

Yokahama (207, S. 67).

$3 \mathrm{Cl}$. planicauda Benedict.

Eine amphiboreale Form (74, S. 1231, 1236; 78, S. 246), Japanisches Meer, 3-6 m, (77, S. 258; 78, S. 172); Florida; Porto Rico (149, S. 404). 
$4 \mathrm{Cl}$. annandalei Tattersall.

Whangpoo River, China, 5-7 m (206, S. 429).

$5 \mathrm{Cl}$. phryganea (Hale).

Süd-Australien (84, S. 321).

$6 \mathrm{Cl}$. tubicola Thomson.

Auckland (216, S. 156); Waijapa Point, Neu-Seeland (35, S. 265).

$7 \mathrm{Cl}$. beathi Richardson.

Monterey-Bai, Kalifornien (149, S. 407).

$8 \mathrm{Cl}$. occidentalis Richardson.

Magdalena-Bai, Kalifornien, 21 m (149, S. 406).

9 Cl. gayi Miers.

Chili (108, S. 82).

$10 \mathrm{Cl}$. linearis Dana.

Valparaiso (136, S. 102); Nord-Patagonien, Rio Negro, aus dem Magen von Silurus spec. (108, S. 81).

$11 \mathrm{Cl}$. natalensis Barnard.

Durban-Bay (9, S. 394).

Puri, Mund des Deviflusses, $14 \mathrm{~m}$; Tanda, $19^{\circ} 52^{\prime}$ N., $86^{\circ} 15^{\prime}$ O., $14 \mathrm{~m}$; Küste Ganjams, $13-17 \mathrm{~m}$, Basseinfluss und Haingyi-Insel, Nieder-Burma (12, S. 186).

$12 \mathrm{Cl}$. granulosa Heller.

St. Paul (95, S. 133); Insel Lennox, Südspitze Süd-Amerika's (139, S. 304); $36^{\circ} 50^{\prime}$ S., $55^{\circ} 54^{\prime} \mathrm{W}$. (136, S. 103).

Die Verbreitung dieser kleinen Gattung ist eine sehr grosse. Ausser den genannten Arten umfasst sie noch angulata (Dana) und danai (Miers) von Rio Janeiro, prismatica (Risso) und whymperi (Miers) nordatlantisch.

Es lässt sich nun bemerken, dass linearis vielleicht identisch ist mit gayi, wenigstens wenn sie wirklich zu Cleantis gehört, was noch zweifelhaft ist $(108$, S. 82). Weiter sind strasseni und heathi eng mit einander verwandt, occidentalis aber schliesst sich mehr der atlantischen planicauda an, während gramulosa und linearis eine südatlantische Gruppe bilden.

\section{Pentidothea Richardson}

\section{Pentidothea japonica (Richardson).}

Japan, auf dem Strande und bis 14 m, Hokio, Rikuoku, Moraran, Hakodate, littoral (146, S. 133; 155, S. 108; 207, S. 63; 147, S. 47) Japanisches Meer, Petrow Insel, etc. 0-6 m. (74, S. 1232; 78, S. 141), eine pazifisch-boreale Art (78, S. 250), lebt nur im littoral.

$2 P$. rotundata Richardson.

Same, Rikuoku, Japan, (150, S. 110); Japanisches Meer, Petrow Insel, 0-3 m. (78, S. 142), kommt in Zostera-Wiesen vor (78, S. 250). Stammt aus den Subtropen (78, S. 245).

$3 P$. australis Hale.

Portland, Victoria, Kangaroo Island (83, S. 318; 84, S. 319). 
4 P. wosnesenski (Brandt).

Ochotskisches Meer; Kamschatka, Bering-Meer; Alaska; Aleuten; Britisch Kolumbien; Kalifornien; littoral bis $17 \mathrm{~m}$. (207, S. 64; 78, S. 143 ).

Nach Gurjanova (74, S. 1235) gehört diese Form mit Idothea aleutica u.a.m. zu den autochthonen amerikanischen Formen, die den Küsten der Aleuten, Kommandor Insel und der Ostküste Kamschatskas entlang verbreitet sind. Daraus erhellt das Vorhandensein einer Verbindung der borealen Meeren mit dem Pazifik (78, S. 239).

5 P. aculeata Stafford.

Kalifornien (180, S. 185).

6 P. whitei (Stimpson).

Puget-Sound, Monterey Bay; Kalifornien (149, S. 373); Nazan Bay, Atka (155, S. 109). Bering-Meer (77, S. 260; 78, S. 144).

Nach Gurjanova (78, S. 239) ist diese Art ein Element der borealen pazifischen Fauna. Sie ist der Hauptsache nach an der amerikanischen Küste verbreitet.

7 P. resecata (Stimpson).

Vancouver; Britisch Kolumbien; Kalifornien, bis 6 m. (149, S. 369); $33^{\circ} 40^{\prime} \mathrm{N} .119^{\circ} 35^{\prime} \mathrm{W}$. (166, S. 364).

8 P. stenops (Benedict).

Monterey Bay, Kalifornien (149, S. 375).

9 P. montereyensis Maloney.

Kalifornische Küste (106, S. 146).

Keine andere Arten sind bekannt.

Die ganze Gattung, deren japonica-whitei-Gruppe sich jedenfalls Idothea ochotensis Brandt ausschliesst, verbreitet sich also über den nördlichen und südlichen Teil des Pazifischen Ozeans und über Australien u.s.w.

Idothea lacustris (Thomson) die vorher bei Pentidothea untergebracht wurde, ist jetzt von Nicholls (124) zu seiner neuen Gattung Notidothea gebracht worden und den Mesidotheinae angegliedert.

Die Gattung Pentidothea liefert, vor allem in der Verbreitung der P. wosnesenski den Beweis, dass zwischen Bering-Meer und Ochotskisches Meer ein Austausch stattfindet, und dass Verbindungen mit dem Stillen Ozean bestehen. Es würde gewiss von Interesse sein, und man wird darauf achten müssen, ob die verschiedenen Tiefseebecken des Niederländisch Indischen Archipels auch einen ähnlichen Faunenaustausch aufzuweisen haben. Das Material ist bisher noch zu dürftig, um weitgehende und sichere Schlüsse ziehen zu können.

\section{Engidothea Barnard}

1 E. lobata (Miers).

St. James, False Bay, Süd Afrika (4, S. 205).

Die einzig bekannte Art. 


\section{Cleantiella Richardson}

1 Cl. isopus (Miers).

Ojica, Insel Goto, $33^{\circ} 12^{\prime} 30^{\prime \prime}$ N., $129^{\circ} 5^{\prime}$ O. (108, S. 81); Hakodate und Mororan, Japan (155, S. 114); Todohokke, Hokkaido und Onagawabucht, 8-10 m. (207, S. 69) Japanisches Meer Insel Petrow (78, S. 173). Nach Gurjanova (78, S. 245) ist diese Art eine littorale subtropische Form.

Die einzig bekannte Art.

\section{Paridothea Stebbing}

1 P. ungulata (Pallas).

Indischer Ozean; Nuyt's Archipelago (45, S. 36); Neu-Seeland; Chatam-Ĩnseln; Süd-Australien; Kangaroo Island; Auckland; Insel Stewart; Insel Campbell; St. Paul; Kap der Guten Hoffnung; Simonstown; Kapstadt; Saldenha-Bai; 9-45 m; Knysna; Chili; Rio Janeiro; Falkland Inseln; (128, S. $133 ; 181$, S. 55. 4, S. $660 ; 64$, S. $436 ; 218$, S. $527 ; 205$, S. $888 ; 83$, S. $318 ; 84$, S. $320 ; 95$, S. 132); Die Varietät atrovirens Collinge; Brighton, Victoria, Australien (56, S. 82).

$2 P$. bakeri Collinge.

Adelaide, Süd-Australien (54, S. 113).

3 P. munda Hale.

Victoria; Neu-Süd-Wales; Süd-Australien; Tasmanien (84, S. 320).

4 P. reticulata Barnard.

Tafelbai; Seapoint bei Kapstadt (6, S. 425).

5 P. rubra Barnard.

Seapoint bei Kapstadt (6, S. 427).

Keine anderen Arten sind bekannt.

\section{Glyptidothea Stebbing}

1 Gl. lichtensteini (Krauss).

Algoa-Bai, 18-29 m. (190, S. 434); Kap der Guten Hoffnung; Tafelbai (108, S. 64).

Die einzig bekannte Art.

\section{Pentias Richardson}

1 P. hayi Richardson.

Misaki, Sagami, Japan (147, S. 48); Hakodate (150, S. 108); Japanisches Meer, Insel Petrow, Tiefe 0-3 m (78, S. 148), nach Gurjanova eine subtropische litorale Form.

2 Pentias thompsoni Collinge.

Yokohama (51, S. 33).

Keine anderen Arten sind bekannt. 


\section{Crabyzos Spence Bate}

1 Crabyzos longicaudatus Spence Bate.

Golf von St. Vincent, 8 m; Süd-Australien: Victoria; Kangaroo Island (108, S. 64; 92, S. 278; 84, S. $319 ; 83$, S. 317).

2 Crabyzos elongatus (Miers).

Süd-Australien; Auckland; $18 \mathrm{~m}$; Neu Seeland; Lyttelton; Akaroa; Falkland-Inseln (108, S. 55; 84, S. $319 ; 216$, S. $156 ; 107$, S. $225 ; 41$, S. $658 ; 197$, S. 369$)$.

3 Crabyzos fucicolus (Barnard).

False Bay an verschiedenen Stellen; Atlantische Küste bei Kap der Guten Hoffnung (6, S. 429).

Keine anderen Arten sind bekannt. Collinge hat aber mitgeteilt, dass er noch 2 unbeschriebene Arten von Süd-Australien besitzt (57, S. 71).

\section{Synischia Hale}

1 Synischia levidensis Hale.

Goif von St. Vincent, Süd-Australien, 11-13 m (81, S. 219; 84, S. 318).

Die einzig bekannte Art.

GRUPPE II VON COLLINGE $\quad(56,5.71)$

Idothea Fabricius ${ }^{1}$ )

1 Idothea baltica (Pallas) (Fig. 47-51).

In seiner trefflichen Zusammenfassung (108) bespricht Mrens ausführlich verschiedene Arten der Gattung Idothea, u.m. I. baltica (Pallas) und die geographische Verbreitung dieser Art. Diese soll sich ausstrecken über die W. Küste Europa's, die Ostsee, Island, die O. Küste N. Amerika's, Brasilien, Mittelmeer, Schwarzes und Kaspisches Meer, Rotes Meer, Java(?) Australien, Neu-Seeland. HANSEN bringt hiergegen Beschwerde nach vorn (88, S. 188); er behauptet, dass Miers vielleicht sechs Arten unter $I$. baltica (von ihm $I$. marina L. genannt) zusammengefasst hat. In der SibogaSammlung nun befinden sich zwei Tiere, welche nach der Form sehr stark mit baltica übereinstimmen, sodass man ohne Studium der Mundteile und Podien, wirklich mit Miers einverstanden sein könnte. Genauere Prüfung der Anhänge aber lehrt, dass in der Tat die Übereinstimmung für eine dieser Formen nicht zutrifft und dass Hansen mit seiner Behauptung nur zum Teil Recht hat.

1 Idothea baltica (Pallas).

Stat. 169. Atjatuning, W. Küste Neu Guinea's, Riffe; 1 Exemplar o’.

Länge $20 \mathrm{~mm}$, Breite $7 \mathrm{~mm}$, Farbe hellbraun.

Das Tier stimmt in den Hauptsachen gut mit baltica (Pallas) überein; nur in einigen kleinen Hinsichten bestehen Unterschiede. So ist die Körperform ein wenig mehr eiförmig und endet das Metasom in einer nicht scharfen Spitze, während Nebenspitzen fast ganz fehlen. So trägt die Antennula am letzten Glied an der lateralen Seite nur 2 Gruppen von 2 keulenförmigen Stacheln

1) Ich schreibe Idothea, weil ich vermute, dass Fabricius mit dem Wahl dieses Namens die Tochter Proteus Eidothea beabsichtigt hat $(185$, S. 51$)$. 
und proximal von diesem eine einzige Stachel. Der Articulus tertius der Maxillula zeigt 9 Stacheln (von welchen 4 gesägt) und 1 spitze Nadel; Collinge (53, S. 738) dagegen gibt an bei baltica 10 Stacheln und $1 \mathrm{Nadel}$; bei baltica ist diese Zahl aber nicht immer konstant und sind von den

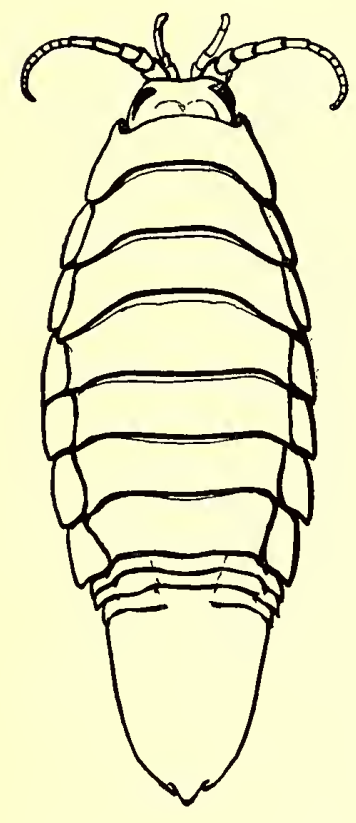

Fig. 47. Idothea baltica (Pallas), $\hat{o} \pm 4 \times$.

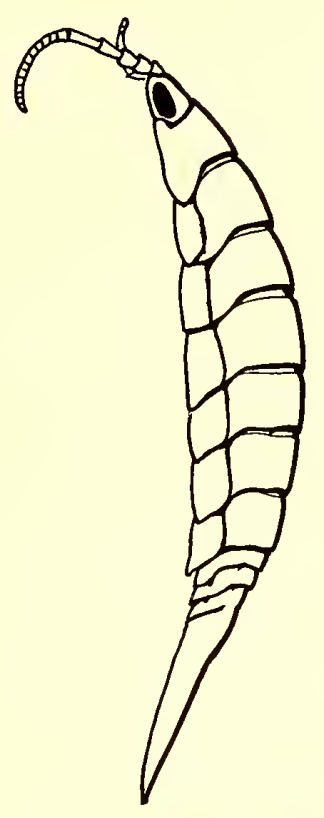

Fig. 48. Idothea baltica (Pallas), ô von der linken Seite, $\pm 4 \times$.

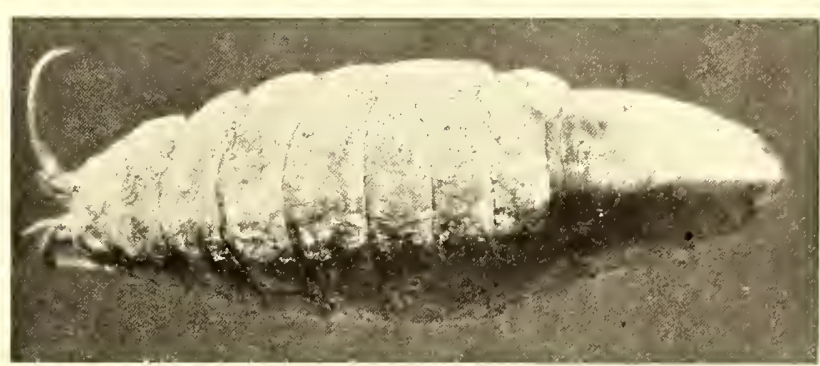

Fig. 49. Idothea balitica (Pallas) ô, $\pm 4 \times$

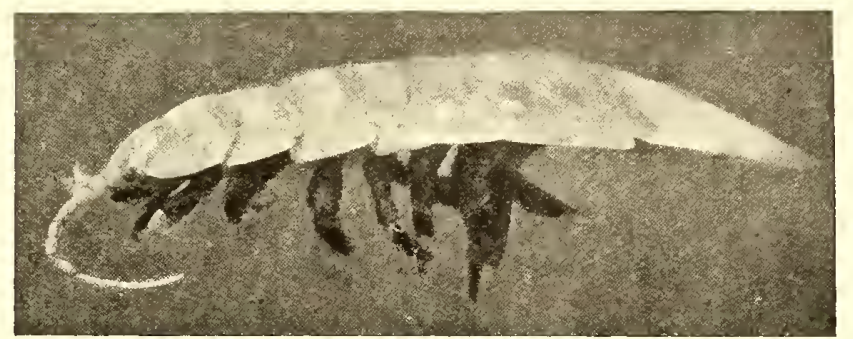

Fig. 50. Idothea baltica (Pallas), ô von der linken Seite, $\pm 4 \times$.

Stacheln immer einige gesägt, was Collinge nicht abbildet. So gibt es mehrere Kleinigkeiten im Bau der Anhänge, z.B. beim Uropod, welches die gefiederte Stachel an der Basis des Endopods nicht zeigt.

Auch muss zugegeben werden, dass das Endglied der Antenna (Fig. 51) etwas anders gestaltet ist, als Collinge (53, S. 738) angibt; Der Endstylus fehlt nämlich und an der Basis findet man mehrere Haare. Überdies betont CollingE besonders, dass dieser Stylus bei den verschiedenen Arten von Idothea eine konstante Form haben soll (53, S. 739).

Diese und die übrigen Unterschiede sind aber m.i. nicht von durchschlagender Bedeutung und ich fühle micht nicht veranlasst eine neue Art zu schaffen; höchstens könnte man von einer lokalen Varietät reden. Denn baltica ist auch in verschiedenen Hinsichten eine variabele Form.

Die geographische Verbreitung von baltica ist dennach sicher eine sehr grosse und grösser, als Hansen (88, S. 188) meint. Die Möglichkeit, dass sie sich bis Neu-Seeland erstrecken soll, ist keineswegs ausgeschlossen, denn HaLE er-

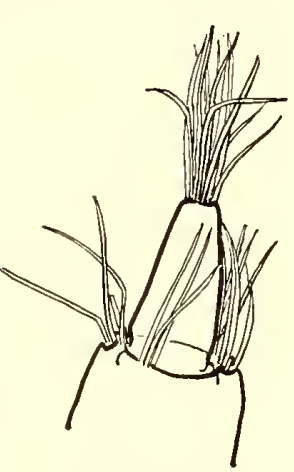

Fig. 51. Idothea baltica, Endglied der Antenna, $160 \times$. wähnt das Vorkommen dieser Art in Australien (81, S. 213), und Thomson \& Chrlton von NeuSeeland (216, S. 155).

Diese Fundorte, sowie Desterro und Rio Janeiro, bleiben aber noch unsicher. Durch das Vorkommen bei Neu-Guinea wird der Fundort Rotes Meer auch wahrscheinlich, da baltica sicher eine Mittelmeerform ist (1, S. 304, 118, S. 407). Nach Stephensen (200, S. 9) ist diese Form häufig in Nord-atlantischen Gewässer, nicht aber in der arktischen Region. 
2 Idothea duplicata nov. spec. (Fig. 52-66).

Stat. 169. Atjatuning, 1 Exemplar $\$$, Westküste Neu-Guinea's, Riffe.

Die Länge beträgt $13 \mathrm{~mm}$, die Breite 31/2 mm. Farbe braungelb. Die Körperform (Fig. 5254 ) ist der von baltica sehr ähnlich. Nur winzige Unterschiede bestehen. Der Körper ist etwas mehr gewölbt als bei baltica, die Antennae sind etwas kürzer; das Metasoma endet in eine kleine nicht scharfe Spitze, während Seitenspitzen kaum angegeben sind (cf. die Varietät basteri Audouin von baltica nach Miers (108, S. 28)). Man muss aber betrachten, dass baltica in ihrer Körperform auch variabel sein kann in mancher Hinsicht. Überdies beschrieb MiERs teils trockene, teils defekte Exemplare.

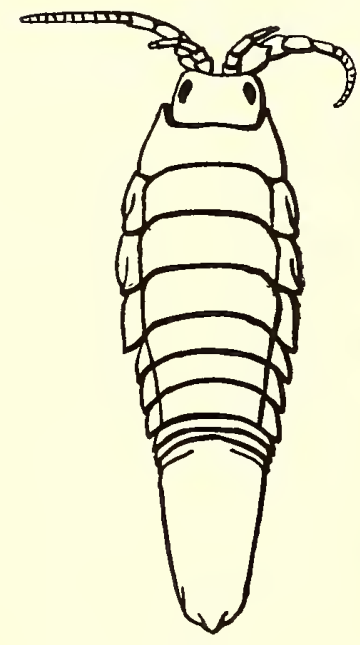

Fig. 52.

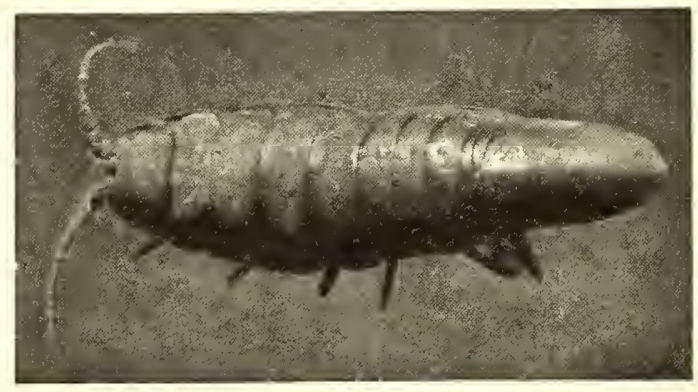

Fig. 53.

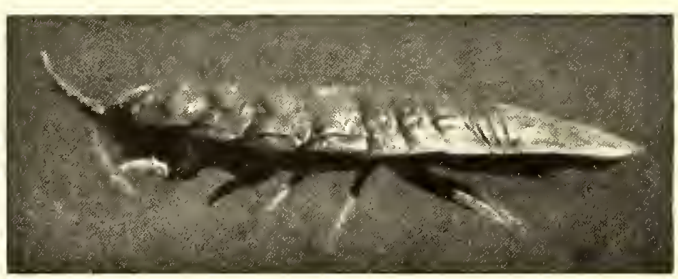

Fig. 54 .

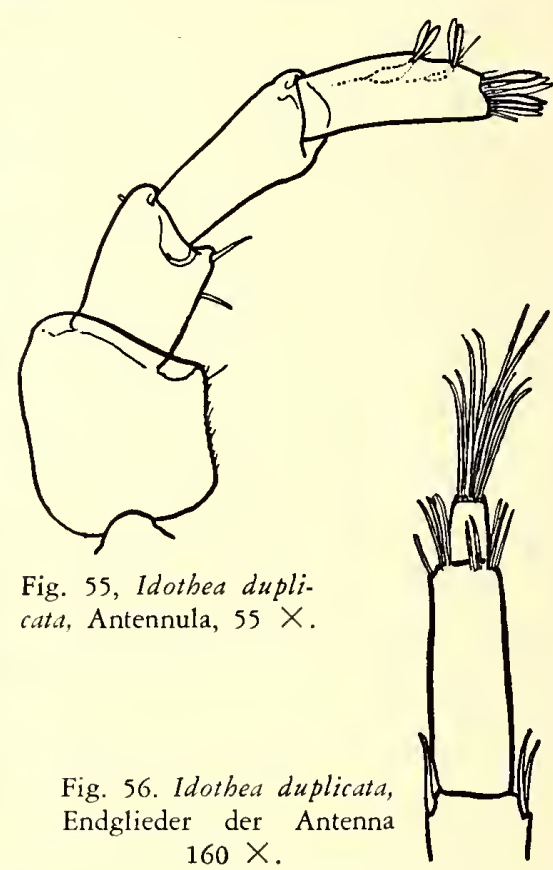

Antennula (Fig. 55): etwas plumper als bei baltica. Das Flagellum trägt terminal eine dorsale Reihe von Läppchen und eine ventrale von 2 spindelförmigen Anhängen und überdies einige spitzen. Tasthaare; die laterale Seite besitzt 2 Gruppen von 2 spindelformigen und einigen spitzen Haare. Nach Collinge (53, S. 738) soll die Antennula von baltica 9-10 Gruppen von ,spatulate setae" zeigen.

Antenna. Flagellum mit 12 Gliedern, von welchen das basale offenbar aus 3 verwachsen ist. Die Zahl soll bei baltica sehr variabel sein, und variieren wenigstens von 12 (149, p. 344 ) bis 20 (56, S. 80,53, S. 738$)$. Das Endglied ist etwas anders von Form als Collinge bei baltica angibt (53, S. 738; Taf. 1, Fig. 3); auch fehlt Collinge's ,terminal style”.

Labrum (Fig. 57). Ein wenig anders als bei baltica; das Basalstiick ist mehr rechteckig abgestutzt und das zweite Stück trägt keine Spitze, aber 2 Reihen von Haaren.

Unterlippe (Fig. 58) schmäler als bei baltica. Sie trägt eine doppelte Reihe von Haaren, eine am Rande und eine mehr basal gelegen. Mandibula (Fig. 59); nur die linke habe ich untersuchen können. Der Bau weicht stark ab von dem bei baltica. Bemerkt muss werden, dass bei dieser Form die Mandibula noch nie genügend beschrieben worden ist. SARS (170, Taf. 32 M) gibt Abbildungen, welche im Allgemeinen richtig, aber für den feineren Bau ungenügend sind. Jedenfalls ist die Mandibula bei duplicata von viel complizierterem Bau. Es fällt aber sehr schwer diesen genau nachzupriifen. Denn an beiden Seiten der Mandibula, also dorsal und ventral, befinden sich Zähne, 
Haare, u.s.w. Bei durchfallendem Licht sieht man alle diesen (Fig. 59); bei schiefer Lichteinstellung ergibt sich aber, dass ein Teil dorsal (Fig. 59) und ein Teil ventral (Fig. 60) liegt. In verschiedenen Hinsichten ähnelt der Bau dem von Cleantis japonica (cf. S. 263).

Maxillula (Fig. 61). Articulus tertius mit etwa 10 terminalen Stacheln, von welchen ver-

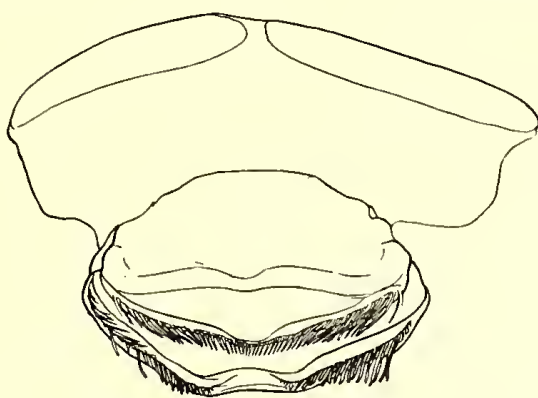

Fig. 57. Idothea duplicata, Labrum, $50 \times$.

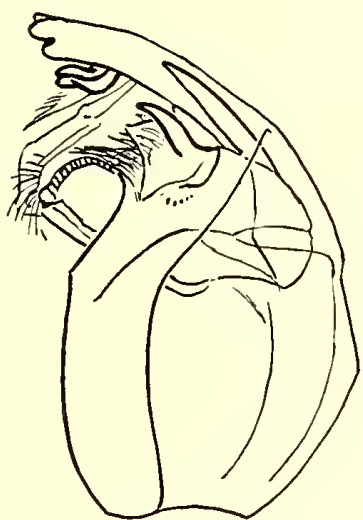

Fig. 60. Idem von der dorsalen Seite.
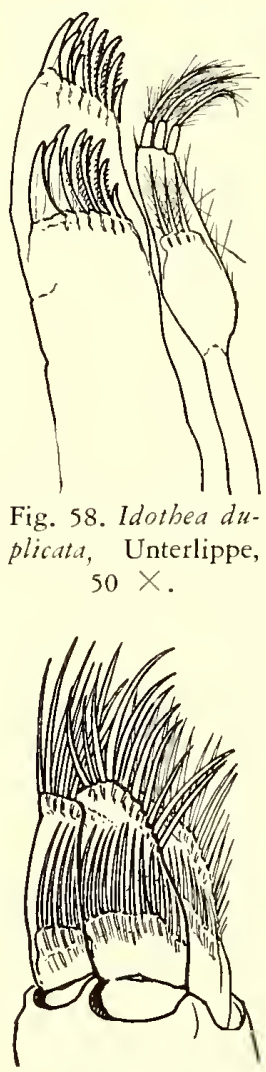

Fig. 62. Idotbed $d u$ plicata. Maxilla, $85 \times$. Die Stacheln sind in Wirklichkeit gekammt.

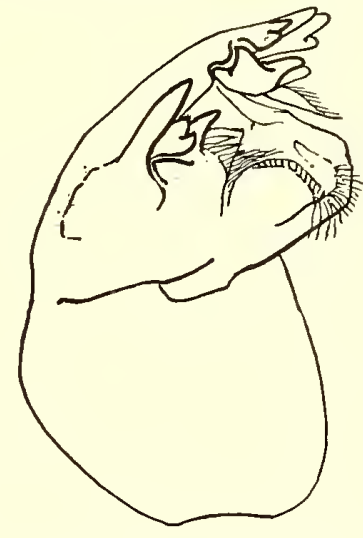

Fig. 59. Idotbea duplicata, linke Mandibula von der ventralen Seite, $55 \times$.

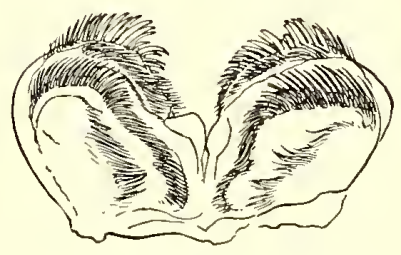

Fig. 61. Idotbea duplicata, Maxillula, $62 \times$

schiedene gesägt. Proximal von diesen eine zweite Reihe von etwa 9 Stacheln. Articulus I mit 3 gefiederten Stacheln wie gewöhnlich, aber auch proximal von diesen kommen noch 3 ähnliche Stacheln vor.

Maxilla (Fig. 62). Articulus tertius und die Lacinia articuli secundi mit zahlreichen feinen Stacheln. Diese Reihen rekapitulieren sich wieder an der proximalen Seite.

Maxillipeden (Fig. 63). Der Habitus ist wie gewöhnlich. Aber auch hier verdoppeln sich alle Reihen von Stacheln.

Thoracopod II (Pereiopod I, Fig. 64). Die Form weicht von der bei baltica ab, cf. CoLLINGE's Fig. 8 (53, Taf. I). Auch hier trägt das letzte Glied eine grosse und kleine Klaue, welche sich proximal rekapitulieren.

Pereiopod II (Fig. 65) ist schlanker als bei baltica (cf. SARS' Fig. p. 2 Taf. 32). Klauenpaare wieder doppelt; dasselbe gilt für Pereiopoden III und IV.

Die Thorakopoden VI, VII und VIII (Pereiopoden V-VII) dagegen haben nur eine Klaue und Nebenklaue, wie normal (Fig. 66).

Pleopoden wie bei baltica.

Uropoden ebenso, aber der Stylus fehlt. 
Diese Form ist bestimmt neu; sie enthält ihren Namen mit Rücksicht auf die ganz eigentümliche Verdoppelung der Reihen von Stacheln an den Mundteilen, welche, soweit ich weiss,

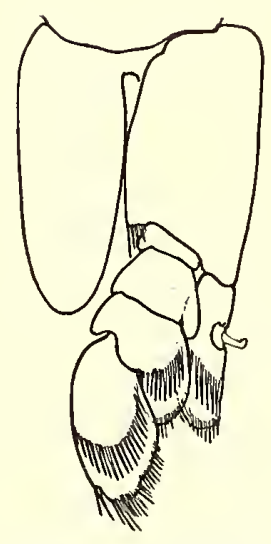

Fig. 63. Idothea duplicata. Maxilliped, $37 \times$.

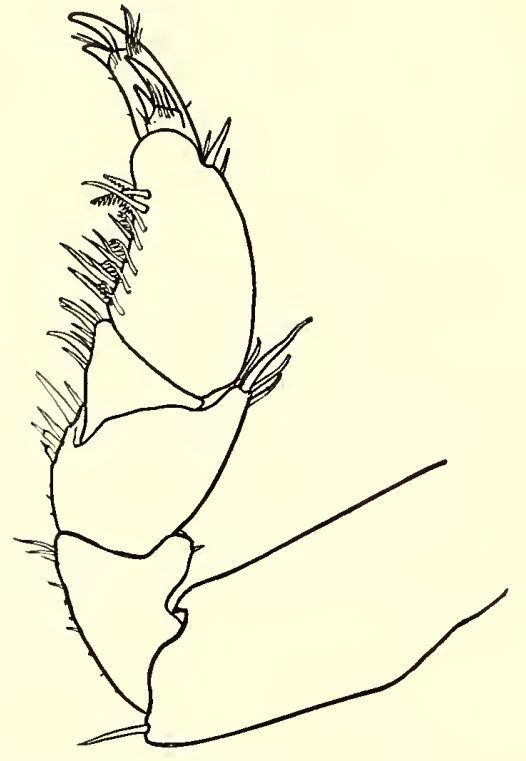

Fig. 64. Idothea duplicata, Thorakopod II (Pereiopod I), $36 \times$.

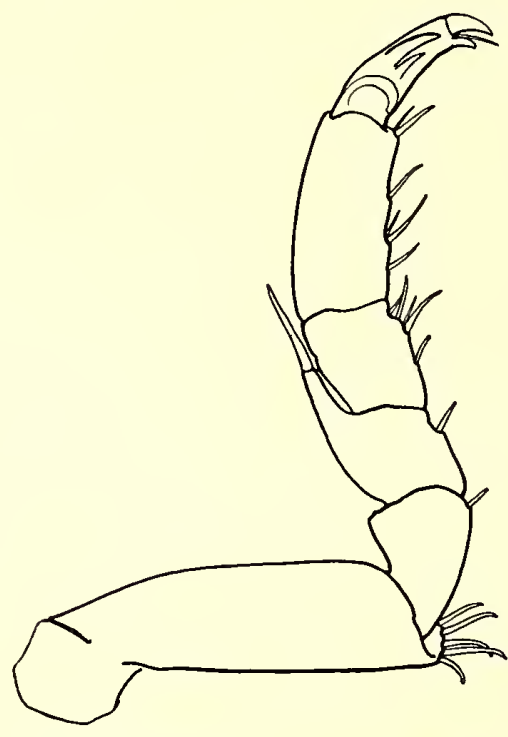

Fig. 65. Idothea duplicata, Thorakopod III (Pereiopod II), $30 \times$.

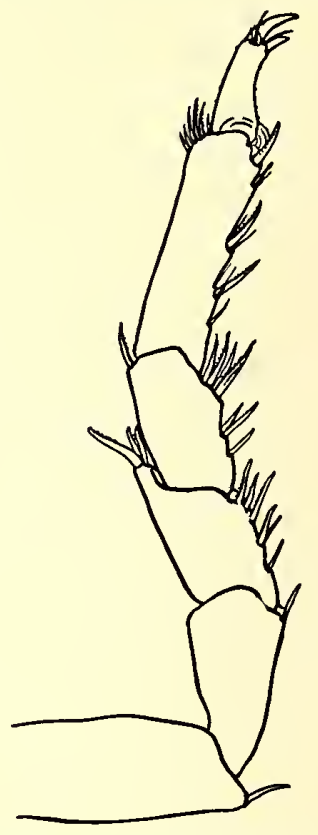

Fig. 66. Idotbea duplicata, Thorakopod VIII (Pereiopod VII), $24 \times$.

nirgends anders vorkommt (vergleiche indessen Cleantis japonica, S. 263). Man könnte vielleicht denken an eine Abnormalität, aber abgesehen hiervon glaube ich doch mit einer neuen aparten Art zu tun zu haben.

3. I. linearis (L.).

Java (?); atlantisch und Mittelmeer; Tiefe bis $22 \mathrm{~m}$ (132, S. 93).

4 I. metallica Bosc.

Borneo; Neu-Süd-Wales; Neu-Seeland; Süd-Pazifischer Ozean; Indischer Ozean; Japan; Cape Point und Mund der Zambesi; Antarktisch (136, S. 94); Magellanstrasse (136, S. 94); nahezu kosmopolitisch; Tiefe bis $1600 \mathrm{~m}$ (108, S. 37; 150, S. 108; 4, S. 203; 218, S. 527; 78, S. 135).

5 I. pustulata (Risso).

Der Fundort dieser Art ist von Miers nicht erwähnt worden. Da sie aber mit metallica eng verwandt zu sein scheint, ist die Möglichkeit keineswegs ausgeschlossen, dass auch hier eine indo-malayische Art vorliegt und will ich sie darum hierbei fügen (108, S. 39).

6 I. caudacuta Haswell.

Griffith's Point; Port Philip; Tasmania (92, S. 182).

7 I. excavata Haswell.

Tasmania (92, S. 183).

8 I. margaritacea (Dana).

Zwischen Australien und Neu-Seeland, 500 Meile von Port Jackson (108, S. 39).

9 I. festiva Chilton.

Sumner bei Christchurch (216, S. 156). 
10 I. ochotensis Brandt.

Ochotskisches Meer; Bering-Meer; Pribiloff Inseln; Britisch Kolumbien; Kalifornien; Alaska; Jeddo; bis $32 \mathrm{~m}(148$, S. 663; 149, S. 366; 147, S. 33; 155, S. 107; 52, S. 83). Nach Gurjanova (74, S. 1234; 78, S. 137) ist es eine autochthone Form der subarktischen asiatischen Seite des Stillen Ozeans; Weitere Fundorte: Kalifornien, Kurillen, Bering-Meer, littoral 0--16 m., Ochotskisches Meer, Japanisches Meer $(78$, S. 136, 137), dort nur im Tartaren Sund $(78$, S. 245). Die Art is euryhalin.

11 I. derjugini Gurjanova.

Eine pazifisch-boreale Form. Bering-Meer, Ochotskisches Meer, Japanisches Meer, Sacchalin, $0-2 \mathrm{~m}$ (74, S. 1230,$1233 ; 77$, S. $259 ; 78$, S. 130$)$.

12 I. aleutica Gurjanova.

Ochotskisches Meer (74, S. 1232, 1233; 77, S. 259; 78, S. 138), Bering-Meer, Aleuten, Kurillen, littoral und sublittoral bis $30 \mathrm{~m}$; Kommandor Insel, 30-50 m; Kamtschatka. Man vergleiche Pentidothea wosnesenskii mit S. nebulosa eine Küstenform des süd-östlichen Teiles des BeringMeeres, die bis zur Westküste von Kamtschatka und dem Ochotskischen Meer durch die KurillenStrasse bei Kap Lopatka durchdringt in dem Bering-Meer. Die Form ist subarktisch.

\section{I. orientalis Gurjanova.}

Bering-Meer, Japanisches Meer, Insel Petrow, 20-25 m; Ochotskisches Meer, 14́-30 m (74, S. 1230; 77, S. 259; 78, S. 140). Es ist diese Art mit I. fewkesi an dem Austansch zwischen Ochotskischen und Japanischen Meer beteiligt ist. Die Art ist pazifisch-boreal. Lebt zwischen Rotalgen.

14 I. urotoma Stimpson.

Puget Sound (149, S. 358).

15 I. gracillima (Dana).

Kalifornien (149, S. 356).

16 I. fewkesi Richardson.

Santa Barbara, Kalifornien (149, S. 360); Bering-Meer, Ochotskisches Meer (78, S. 136), Tiefe 0-18 m. Japanisches Meer, 0-8 m, (74, S. 1230, 1234; 77, S. 258; 78, S. 136); eine südliche subtropische Form, die aus dem japanischen Meer in das Ochotskische Meer durch den Nevelski-Sund durchdringt. Die Art is wärmeliebend.

17 I. rectilineata Lockington.

Kalifornien, 54--72 m (149, S. 360).

18 I. rufescens Fee.

Departure Bay (63, S. 30).

19 I. delfini Porter.

Coquimbo' 142, S. 153).

20 I. indica $\mathrm{H}$. Milne Edwards.

Malabar (110, S. 132); Hout Bay, 16-36 m (184, S. 63); Tabalbai, 18 m (127, S. 111, S. 129).

21 I. hectica (Pallas).

Bourbon; Mittelmeer, Atlantik (48, S. 161). 
Es fällt auf, dass im Indo-malayischen Archipel nur 4 Arten vorkommen, vielleicht 5, von welchen 2 als Kosmopoliten zu betrachten sind und auch die dritte sich über ein grösseres Gebiet erstreckt. Von den Philippinen sind gar keine Arten beschrieben worden. Die metallica-pustulataGruppe ist mit der mehr südlichen, subantarktischen margaritacea verwandt, marina dagegen mit der nördlichen ochotensis, welche Form im ganzen nördlichen Pazifik vorkommt, aber autochthon ist für die asiatische Seite des Pazifiks (74, S. 1235); linearis schliesst sich mehr der westlichen indica an.

Von den etwa 39 bekannten Arten von Idothea kommen also etwa 20 im indo-pazifischen Gebiet vor. Die Australien-Neu-Seeland-Gruppe umfasst, ausser den kosmopolitischen baltica(?) und metallica 4 Arten.

Die nord-pazifische Gruppe umfasst ocbotensis, urotoma, gracilimma, fewkesi, rectilinea, derjugini. aleuticus und orientalis. Von diesen ist ochotensis mit der nord-atlantischen phosphorea Harger verwandt. Die enge Übereinstimmung mit japonica Richardson, welche nach THIELEMANN bestehen sollte (207, S. 63), ist offenbar nicht vorhanden, weil diese Form mit ihren fünfgliedrigen Maxillipeden zu Pentidotea gerechnet werden muss (207, S. 108), während ochotensis viergliedrige Maxillipeden besitzt (149, S. 366). Diese Übereinstimmung ist also nur scheinbar; ohne Vergleich der Mundteile ist es unmöglich eine gute Systematik der Isopoden aufzustellen. Übrigens stehen urotoma, fewkesi und rectilinea einander nahe. Derjugingi, aleuticus und orientalis sind mir leider unbekannt.

Weil PorTer's Beschreibung (12) mir nicht zur Verfügung stand, ist delfini mir unbekannt. Sehr arm an Formen ist der Indische Ozean, denn nur indica kommt in diesem Gebiet mit Sicherheit vor; sie ist verwandt mit den atlantischen emarginata, linearis und bectica. Letztgenannte Form soll nach MIERs bei Bourbon vorkommen; meint er hiermit die grösste Insel der Maskarenen, so ist auch bectica eine indische Form. Und weil auch von linearis das Vorkommen im Indischen Ozean bis Java erwähnt worden ist, so haben wir es hier vielleicht mit einer atlantischen Gruppe zu tun, welche sich ostwärts verbreitet.

\section{Euidothea Collinge}

1 Euidothea peroni (H. Milne Edwards).

Australien; York's Halbinsel; Flinders Island, Bass-Strasse; King's' Insel; Melbourne; Kangaroo Island; Süd-Australien, 4-5 m (110, S. 133; 108, S. 56; 83, S. 315); Chatham-Inseln (38, S. 269); Kap der Guten Hoffnung (190, S. 433).

2 Euidothea stricta (Dana).

Neu-Süd-Wales (108, S. 63); Golf von St. Vincent, Süd-Australien, 11-13 m; Kangaroo Island (81, S. $214 ; 84$, S. 316$)$.

3 Euidothea bakeri Collinge.

Victoria; Süd-Australien; Kangaroo Island (83, S. 315; 81, S. 215; 84, S. 317).

4 Euidothea coeruleotincta Hale.

Kangaroo Island, Süd·Australien (83, S. 317).

Die einzig bekannten Arten. 
Colidothea Richardson

1 C. rostrata (Benedict).

San Pedro, Kalifornien (149, S. 393).

Die einzig bekannte Art.

Eusymmerus Richardson

1 E. antennatus Richardson.

Abreojos Point, Unter-Kalifornien, 10 m (149, S. 399).

Die einzig bekannte Art.

Erichsonella Benedict

1 Erichsonella pseudoculata Boone.

Kalifornien (22, S. 154).

2 E. nordenskjöldi Ohlin.

Patagonien (139, S. 304).

Diese Gattung zählt 5 Arten, von denen 3, attenuata (Harger), filiformis (Say) und floridana Benedict, atlantisch sind.

\section{Synisoma Collinge}

Die Gattung Synisoma ColLINGE enthält keine indopazifische Vertreter.

\section{GRUPPE II I VON COLLINGE (56, S. 71)}

\section{Edotia Guérin-Ménéville}

1 E. dilatata Thomson.

Auckland (211, S. 235).

2 E. magellanica Cunningham.

Kap Espiritu Santo, Strasse von Magellan (108, S. 74; 67, S. 12; 136, S. 98); West Patagonien (139, S. 296).

3 E. tuberculata Guérin-Ménéville.

Chili; Magellan-Strasse; Cap Hoorn; Feuerland; Falkland-Inseln; Süd-Georgien, 5-310 m (136, S. $96 ; 139$, S. $294 ; 60$, S. $70 ; 108$, S. $73 ; 182 ;$ S. $353 ; 67$, S. 12 ).

4 E. lilljeborgi Ohlin.

West-Patagonien (139, S. 298).

5 E. doello-juradoi Giambiagi.

Insel Dawson, Feuerland (67, S. 14).

Die übrigen Arten dieser Gattung, triloba (Say), montosa (Stimpson) und acuta Richardson sind nord-atlantisch, oculata Ohlin und bilobata Nordenstam sind süd-atlantisch. 
Es fällt auf, dass der grösste Teil der angeführten Arten von der Südspitze Süd-Amerika's stammt und höchstwahrscheinlich als atlantisch anzusehen ist. So bleiben nur dilatata und tuberculata als pazifische Formen übrig. Ob aber dilatata zu Edotia gehört ist zweifelhaft; THomson selbst behauptet, es stehe diese Form zwischen Idothea und Edotia ein. Das Vorkommen von Antennen mit 13-14 Gliedern weist aber nicht auf Zusammenhang mit Edotia, bei welcher Gattung das Flagellum der Antennen rudimentär ist.

Und auch tuberculata kann nicht mit Sicherheit des Vorkommens an der Küste Chili's zu den pazifischen Formen gerechnet werden, denn diese Tatsache beruht hierauf, dass Epelys annulatus Dana und Desmarestia chilensis Nicolet von OHLin als Jugendformen von tuberculata angesehen werden (139, S. 283), welche Auffassung aber nicht erwiesen ist (ich glaube aber, dass OHLIN Recht hat, und habe deshalb beide Formen bei tuberculata untergebracht). Im ganzen kann man aber jedenfalls dies behaupten, dass Edotia eine atlantische Gattung ist, welche höchstens eine geringe westliche Verbreitung in den Pazifik erlangt hat.

\section{Synidothea Harger}

1 S. epimerata Richardson.

$43^{\circ} 19^{\prime} 20^{\prime \prime}$ N. $140^{\circ} 17^{\prime}$ O., Japan $106 \mathrm{~m}$ (150, S. 113; 78, S. 162), Japanisches Meer 20-80 m, Insel Petrow, Amerika; Lebt auf steinigem Boden.

2 S. laevidorsalis Miers.

Yokohama (207, S. 65); Nord-Pazifischer Ozean, $39^{\circ} 2^{\prime}$ N. $189^{\circ} 50^{\prime}$ O., $12 \mathrm{~m}$ (108, S. 69, 15, S. 403).

3 S. tuberculata Richardson.

Insel Sachalin, $48^{\circ} 32^{\prime} 45^{\prime \prime}$ N. $145^{\circ} 07^{\prime} 30^{\prime \prime}$ O., $48^{\circ} 32^{\prime} 30^{\prime \prime}$ N. $145^{\circ} 08^{\prime} 45^{\prime \prime}$ O., Flat Hill; Kap Patience, $48^{\circ} 43^{\prime} 30^{\prime \prime}$ N. $145^{\circ} 03^{\prime}$ O. und $48^{\circ} 43^{\prime} 10^{\prime \prime}$ N. $144^{\circ} 59^{\prime} 30^{\prime \prime}$ O., 121-135 m. (150, S. 114); Ochotskisches Meer 120-135 m (77, S. 259; 78, S. 159).

4 S. longicirra Gurjanova.

Ochotskisches Meer, Tiefe 30-141 m. (77, S. 259; 78, S. 160).

5 S. cinerea Gurjanova.

Ochotskisches Meer, 22 m, (78, S. 170; 77, S. 259).

O S. spinosa Gurjanova.

Bering-Meer, 88-95 m. Ochotskisches Meer, 14-74 m (74, S. 1230; 77, S. 259, 260; 78, S. 168).

7 S. braznikovi Gurjanova.

Japanisches Meer 5-25 m, (77, S. 258; 78, S. 157); eine Form der Zosterawiesen.

8 S. excavata Gurjanova.

Japanisches Meer (77, S. 258).

9 S. pacifica Nobili.

Mangareva, Tuamotu-Inseln (135, S. 420). 
10 S. erosa Benedict.

Insel Sannakh, Alaska, 869 m (15, S. 379); Bering-Meer (77, S. 260; 78, S. 158).

11 S. pallida Benedict.

Insel Chirikof, Alaska, 1251 m (15, S. 397); Bering-Meer, Tiefe bis 1380 m (77, S. 260; 138, S. 158).

12 S. nebulosa Benedict.

Unalaska; Kyska Harbour; Semidi-Inseln; Insel Unimak; Bering-Meer; Kamschatka; Constantine Harbor, Alaska; Insel Amak; 11-58 m (149, S. 381); Bering-Meer; Ochotskisches Meer (74, S. 1233; 77, S. 259; 78, S. 156).

13 S. laevis Benedict.

Zwischen Bristol Bay und den Pribiloff-Inseln, Alaska; Bering-Meer; 53-65 m (149, S. 389), 52 m (74, S. 1230; S. 166).

14 S. macginitiai Maloney.

Kalifornien (106, S. 144).

15 S. picta Benedict.

Alaska; Beringstrasse; Norton Sund 9-36 m (149, S. 391; 78, S. 169); Bering-Meer.

16 S. bicuspida (Owen).

$73^{\circ}, 52^{\prime}-75^{\circ} 31^{\prime} \mathrm{N} ., 78^{\circ} 20^{\prime}-124^{\circ} 30^{\prime}$ O., Tiefe $19-40 \mathrm{~m}(75, \mathrm{~S} .31-34) ; 76^{\circ} 38^{\prime} \mathrm{N} .81^{\circ}$ $41^{\prime}$ O. Tiefe 57 m (76, S. 596), eine östliche arktische Form Westküste Alaska's; Bering-Meer; Karisches Meer, 6-146 m. (149, S. 385); von Spitzbergen bis der Beringstrasse und südwärts bis an den Golf von St. Lawrence; Matyushin Shar (108, S. 67). Es ist dies eine Arktische Form, die in dem Bering-Meer eindringt. Siberisches Meer, nord-atlantisch und arktisch (108, S. 28) BeringMeer (78, S. 153), Pribiloff Insel, Unimak, 64-88 m (Confer 77, S. 1230). Die Varietät lata Gurjanova im Japanischen Meer; Ochotskisches Meer, 7 m; Bering-Meer 5-20 m (77, S. 258, 259).

17 S. acuta Richardson.

Bering-Meer; Insel Semisopochni, $52^{\circ} 12^{\prime}$ N. $179^{\circ} 52^{\prime}$ O., 86 m (150, S. 111; 78, S. 161),

18 S. marmorata (Packard).

Atlantische Ozean, Labrador, 14 m (149, S. 384), Bering-Meer, 81 m (77, S. 260; 78, S. 155). Mit S. bicuspida und S. nodulosa eine arktische Form, die in dem Bering-Meer eindringt.

19 S. muricata (Harford).

$41^{\circ} 3^{\prime}$ N. $154^{\circ} 15^{\prime}$ W.; Icy Cape, $70^{\circ} 15^{\prime}$ N. $162^{\circ} 55^{\prime}$ W., 45 m (149, S. 390).

Ochotskisches Meer, 16-100 m (77, S. 259; 78, S. 167; 74, S. 1230). Es ist dies ursprünglich eine pazifische Form, die erst später in dem Beaufort-Meer gelangt ist und von dort nach Westen nach dem Karischen Meer vorgedrungen ist.

20 S. nodulosa (Kröyer).

Pazifisch südwarts bis Britisch Kolumbien; Alaska; Grönland; Spitzbergen; arktisch, Aleuten; circumpolar, 5-199 m (149, S. 388; 88, S. 191; 74, S. 1232; 78, S. 165), eine arktische Form.

21 S. angulata Benedict.

$47^{\circ} 38^{\prime}$ N. $124^{\circ} 39^{\prime}$ W.; Kap Johnson; Insel Destruction; Kap Flattery; Washington, 59-68 m $(149$, S. 382; 15, S. 396)

22 S. laticauda Benedict.

Bai von San Francisco (15, S. 393). 
23 S. harfordi Benedict.

Magdalena-Bai; San Diego-Bai; Kalifornien 12 m (149, S. 387); Japanisches Meer, Insel Petrow, 0-10 m (77, S. 258; 78, S. 164; 74, S. 123), eine wärmeliebende subtropische, kalifornische Art, die auch im Tartaren Sund vorkommt.

24 S. consolidata (Stimpson).

Pacific Grove, Kalifornien (15, S. 393).

25 S. ritteri Richardson.

San Francisco, Kalifornien (149, S. 377).

26 S. variegata Collinge.

Golf von Manaar (55, S. 1); Indo China (120, S. 6); Chilka-See bis 5 m (46, S. 891); Küste von Ganjam, 13-17 m (12, S. 185); Manumbam, Travancore (11, S. 313).

27 S. setifera Barnard. $33^{\circ} 3^{\prime}$ S. $27^{\circ} 57^{\prime}$ O., $58 \mathrm{~m}(4$, S. 206).

28 S. hirtipes (Milne Edwards).

Simonsbai; Kap der Guten Hoffnung; 7-13 m (108, S. 69; 15, S. 403); Camerun (117, S. 2); Kap St. Blaize (190, S. 434; 184, S. 62); Saldanha-Bai 9-45 m (204, S. 888); Suezkanal (140, S. 205); Abdelkuri (221, S. 219), Kap Barracouta, bis 72 m; Sebastian Bay; Walker Bay, (196, S. 3). Von den 29 bekannten Arten von Synidothea sind also nicht weniger als 28 dem Indo-pazifischen Gebiet zuzurechnen. Nur marplatensis Giambiagi ist rein atlantische Form (149, p. 392; 66, S. 240).

Im Indo-malayischen Archipel ist noch keine Art gefunden worden; auch in Australien scheinen sie zu fehlen.

Der grösste Teil der etwa 25 Synidothea-Arten ist im nördlichen Pazifik gefunden, und von dort in dem Bering-Meer, in dem Ochotskischen Meer und in dem Japanischen Meer durchgedrungen. Pazifische Formen dringen durch die Beringstrasse in das Eismeer und wenden sich dann, entweder an der Siberischen Küste entlang durch nach dem Westen (S. bicuspida, S. nebulosa) während andere Formen, so S. marmorata, nach Osten in die Beaufort-See gelangen und von hier aus weiter nach dem Westen bis zu den Farör durchdringen.

\section{Lyidothea Hale}

1 Lyidothea nodata Hale.

Nord-Queensland: Flinders Island, Princess Charlotte Bay (84, S. 36).

Die einzig bekannte Art.

\section{Subfamilie Glyptonotinae \\ Symmius Richardson}

1 S. caudatus Richardson.

Ose Zaki, Japan, 108--126 m (147, S. 41; 78, S. 150).

Die einzig bekannte Art.

Die verwandte Gattung Glyptonotus Fights mit der einzigen Art antarcticus Eights und deren Varietät acutus Richardson ist antarktisch und süd-atlantisch. 


\section{Subfamilie Macrochiridotheinae}

\section{Macrochiridothea Ohlin}

1 M. michaelseni Ohlin.

Magellan-Strasse, Punta Arenas 22 m; in der Delta des Rio de las Minas, 32 m (139, S. 289).

2 M. stebbingi Ohlin.

Zwischen Isla Nuova und Navarino; Südspitze Süd-Amerika's 54 m (139, S. 291); Port Harriet;

Falkland-Inseln (193, S. 354).

Die Varietät multituberculata Nordenstam: Falkland-Inseln (136, S. 112).

3 M. kruimeli Nierstrasz.

Punta Arenas (128, S. 130).

Keine anderen Arten sind bekannt.

\section{Chaetilia Dana}

1 Ch. orata Dana.

Rio Negro, Patagonien (65, S. 263; 58, S. 713).

Eine dritse Gattung Chiriscus Richardson mit nur einer Art kommt vor beim Rio de la Plata, Argentinien, $21 \mathrm{~m}$ (160, S. 171).

Die Gruppe ist also als süd-atlantisch und antarktisch anzusehen.

\section{Subfamilie Mesidotheinae}

\section{Mesidothea Richardson}

1 Mesidothea entomon (L.)

Kalifornien: Westküste Nord-Amerika's (149, S. 348); Alaska, Petrapolovsk (156, S. 107), selır allgemein; Nord-Atlantik; Arktische Küste Kanadas; King William Land; fehlt in den Gewässern rund Grönland und Island, nur bei Spitzbergen (199, S. 12), arktisch circumpolar; Ostsee; Mälar-, Wettern-, Mjösen-, Ladoga- und Onega-See. Vergleiche hierbei das Vorkommen von Notidothea lacustris (Thomson) S. 272; Kaspisee; Aralsee, bis 95 m (132, S. 94; 70, S. 239); Eismeer (74, S. 1.230). Die subspecies orientalis Gurjanova lebt im Bering-Meer, Ochotskisches Meer, Japanisches Meer, u.s.w. (78, S. 147); entomon Gurjanova in der Ostsee, Weisses Meer; glacialis Gurjanova im Nord Polarmeer, auch im Kaspischen Meer.

Die Varietät vetterensis Ekman im Kaspischen Meer (61, S. 259). Die von STEPHEnsen (199, S. 12) bei King Williams Land gefundenen Exemplare sind derjenigen der subspec. glacialis Gurjanova am meisten ähnlich.

\section{Mesidohea sabini (Kröyer)}

Alaska, arktisch circumpolar, 7-475 m (149, S. 351; 69, S. 310; 76, S. 596; 88, S. 186).

Die typische Form $M$. sabini wurde von Gurjanova (78, S. 31-34; 76, S. 587-593) fast an allen Stationen angetroffen. Sie war anwesend von $76^{\circ} 30^{\prime} \mathrm{N} .-80^{\circ} 26^{\prime} \mathrm{N}, 70^{\circ}-105^{\circ} 38^{\prime} \mathrm{O}$ bei einer Tiefe von 43-475 m. StePhensen (199, S. 3) fand seine Stücke an der Middleton Küste, Roe's Wellcome, Roe's Welcome Insel und Anarnigtoq, Roe's Wellcome. Die subspecies robusta Gurjanova ist eine östliche hocharktische Form, $78^{\circ} 58^{\prime} \mathrm{N}, 68^{\circ} 25^{\prime} \mathrm{O}(76, \mathrm{~S} .597)$ und von $74^{\circ}$ 
$05^{\prime}-71^{\circ} 39^{\prime} \mathrm{N}, 79^{\circ} 08^{\prime}-124^{\circ} 30^{\prime} \mathrm{O}$, Tiefe $24-42 \mathrm{~m}$. Grösste Tiefe der Art, bis $350 \mathrm{~m}$ (72, S. $444 ; 76$, S. 597).

Noch zwei Arten sind bekannt: megalura (Sars), nord-atlantisch und arktisch, Tiefseeform, und sibirica (Birula), nord-asiatisch und arktisch.

Die ganze Gattung ist derhalb grösstenteils hochnordisch. Die Subfamilie selbst is sehr alt, denn die zugehörige Proidothea haugi Racovitza \& Sevastos stammt aus dem Oligocän der Karpathen (143, S. 194). Die rezente Gattung Chiridothea Harger mit 2 Arten ist atlantisch.

\section{Austridotea Nicholls}

1 Austridotea annectens NichOLLS.

Stewart Insel, Neu Seeland, in Süsswasser (124, S. 118).

2 Austridotea benbami Nicholls.

Stewart Insel (124, S. 123), Neu Seeland (34, S. 131; 35, S. 263), Otago Peninsula, Port Chalmers, Dunedin, Hafen (50, S. 154) Waitiki, Blueskin Bay (41, S. 658).

Nach Nicholis (124, S. 125) ist es anzuzweifeln, dass die von Chilton (41, S. 658; 47, S. 319) auf den Campbell Insel gefundenen Exemplaren, die zu einer Varietät von der ursprünglich als Pentidothea lacustris (Thomson) bezeichneten Form, welche hier als Austridotea benhami vorgeführt wird, gebracht wurden, mit dieser Form conspezifisch ist. Ihre Identität muss, bis weitere Untersuchungen vorliegen, fraglich bleiben.

\section{Notidothea Nicholls}

Ursprünglich als Untergattung von Austridothea aufgestellt, möchte ich diese Formengruppe lieber als Gattung würdigen (S.S.).

Es gehören 2 Arten dazu:

1 Notidothea lacustris (G. M. Thomson).

Der grossen Verwirrung wegen, die in der Identifikation dieser Art vorherrschte und dic jetzt grösstenteils durch die Arbeit von Nicholls behoben wurde, macht es nichtdestoweniger eine sehr eiklige Sache die verschiedene für Idothea lacustris G. M. Thomson angegebene Fundorte auf ihre Zuverlässigkeit zu prïfen, umsomehr, da lokale Formen für I. lacustris Thomson beschrieben worden sind, die jetzt über Notidotea lacustris und $N$. rotundicauda somit über Austridothea verteilt werden. Ich werde mich daher notgezwungen soviel tunlich an den Angaben Nicholls halten.

Tomahawk lagoon, bei Dunedin, Süsswasser (209, S. 250); Neu-Seeland (35, S. 263); Mt. Mihiwaka; Otago; Hafen von Dunedin (50, S. 155); Chatham Inseln; Insel Campbell (41, S. 658; 47, S. 319); Porto Low; Golf von Corcovado; West-Chile, Brackwasser (128, S. 130).

Das Vorkommen dieser Form in süssem und mehr salzigem Wasser oder eben im Meere ist fremdartig. Darauf hat VAN NAmE (279, S. 451) bereits aufmerksam gemacht. Ein paralleler Fall liegt bei Mesidothea entomon vor. Vermutlich haben wir es hier bei $N$. lacustris mit einer Süsswasserart von mariner Herkunft zu tun, die sehr tolerant für Salzwasser, also euryhalin ist.

$2 N$. rotundicauda (Miers).

Neu-Seeland (124, S. 131; 33, S. 194); Port Henry; Magellanstrasse (108, S. 40; 41, S. 659). 
Familie Holognathidae

\section{Holognathus G. M. Thomson}

1 Holognatbus stewarti (Filhol).

Insel Stewart (64, S. 492); Timaru, $72 \mathrm{~m}$ und Waganui, Neu-Seeland (215, S. 67).

Die einzige Art.

\section{Unterordnung ASELI.OTA \\ Familie Stenetriddat \\ Stenetrium Haswell}

1 Stenetrium siamense Hansen.

Golf von Siam, zwischen Koh Mesan und Koh Chuen, 27-68 m (87, S. 329).

2 Stenetrium chiltoni Stebbing.

Galle, Ceylon; Golf von Manaar (188, S. 56); Amirante, Indischer Ozean 61 m (191, S. 110); Mittelmeer? (106); Golf von Suez (119, S. 171).

3 Stenetrium machocheir Nicholls.

Dongarra, West-Australien (123, S. 363).

4 Stenetrium spinirostrum Nicholls.

Rottnest Island, West-Australien (123, S. 369).

5 Stenetrium truncatum Nicholls.

Rottnest Island, West-Australien (123, S. 371).

6 Stenetrium glauerti Nicholls.

Rottnest Island, West-Australien (123, S. 373).

7 Stenetrium armatum Haswell.

Port Jackson, Port Stephens; Griffiths' Point, Victoria (92, S. 309); Süd-Australien (84, S. 325).

8 Stenetrium fractum Chilton.

Lyttelton Harbour, Neu-Seeland (87, S. 249; 32, S. 250).

9 Stenetrium hanseni Nobili.

Fakahina, Paumotu-Inseln (135. S. 414).

10. Stenetrium euchirum Nobili.

Tearia und Gatavakè, Paumotu-Inseln(?) 20 m. (135, S. 415).

11 Stenetrium proximum Nobili.

Vahitahi; Paumotu-Inseln(?) (135, S. 416).

12 Stenetrium crassimanus Barnard.

St. James, False-Bay (4, S. 219).

13 Stenetrinm dagama Barnard.

Vasco da Gama Peak, 414 m; Table mountain, 342 m (7, S. 400). 
14 Stenetrium dalmeida Barnard.

Lion's Head, 414 m; Cape Point, 243 m (7, S. 401).

15 Stenetrium diazi Barnard.

Buffel's Bay (7, S. 402).

16 Stenetrium saldanha Barnard.

Kap St. Blaize, 225 m; Cape Point, 243 m (7, S. 404).

Im ganzen sind 24 Arten beschrieben worden, von welchen also 8 nicht zum Indo-pazifischen Gєbiet gehören. Von genannten 8 Arten kommen 4 in West-Indien vor (stebbingi Richardson, serratum, Hansen, occidentale Hansen und antillense Hansen; St. haswelli Beddard ist von der Challenger-Expedition gefunden worden $37^{\circ} 17^{\prime} \mathrm{S}$. $53^{\circ} 52^{\prime} \mathrm{W}$. in $1080 \mathrm{~m}$ und VANHöFfen gibt 2 Formen, St. acutum und rotundatum, von der Gauss-Station der deutschen Südpolar-Expedition 1901-1903 (218, S. 546, 548); wenn ich richtig gehe, meint VANHÖFfEN hiermit das Winterstation dieser Expedition, $66^{\circ} 2^{\prime}$ S. $89^{\circ} 38^{\prime}$ W., 385 m, also auf der Grenze des antarktischen Gebietes; diese Form ist auch von Süd-Georgien, 252-310 m, bekannt (136, S. 276). Schliesslich bleibt noch St. mediterraneum Hansen aus dem Mittelmeer. Im Indo-australischen Archipel und den Philippinen sind noch keine Vertreter gefunden worden, ebensowenig im Nord-Atlantik und arktisch. Fremd ist die Verwandtschaft von acutum mit occidentale Hansen, stebbingi Richardson und antillense Hansen, alle aus West-Indien.

\section{Familie Parasellidae \\ Gruppe Ianirini \\ Ianira Leach}

Es herrscht eine grosse Verwirrung in Bezug auf diese Gattung, von welcher TatTERSALL (205, S. 199) und Nordenstam (136, S. 171) eine gute Übersicht geben. Es ist natürlich Geschmacksache, ob man Ianthopsis Beddard und Iolella Richardson als gesonderte Gattungen stehen lassen will, wäre es nur des Umstandes wegen, dass hierdurch die grosse Gattung Ianira eingeengt würde. Das Prinzip der Einteilung in den 3 Gattungen Ianira, Ianthopsis und Iolella stutzt sich auf das Vorkommen der Epimeren, welche entweder an allen Segmenten vorhanden sein können (Ianira), oder an den letzten drei Segmenten - wenigstens fehlen sie bei den freien Segmenten II und III - gefunden werden (Iolella) oder ganz fehlen (Ianthopsis) (205, S. 199), und auf die Form des Metosoms (218, S. 536). Mit Recht bemerkt Nordenstam (136, S. 177), dass, wenn man die genannte Einteilung annehmen will, die bis jetzt zu Iolella gerechneten Arten einer Revision bedürfen; dasselbe gilt aber auch für Ianthopsis. In vielen Fällen ist es sogar unmöglich zu entscheiden, zu welcher Gattung eine Art gerechnet werden muss. Ich gebe hier einen kleinen Auswahl solcher Fällen.

Ianira abyssicola Beddard; von dieser Art sind die Epimeren ganz unbekannt (13, S. 6).

Ianira angusta Barnard; Epimeren werden nicht genannt. Barnard's Fig. I, Taf. 17 (7) zeigt keine Spur von diesen. Diese Form könnte deshalb in dieser Hinsicht eine Iolella sein, zeigt sich aber in der Bildung des Metasoms eine Ianira.

Ianira caudata Richardson zeigt keine Spur von Epimeren; das Metasom dagegen ist Ianthopsis-ähnlich (158, S. 33). 
Stebbing's Fig. A I und D, Taf. 6A (191), von Ianira crosslandi zeigen Epimeren an den zweiten, dritten und vierten freien Mesomeren; diese Form weicht also in Bezug auf die Epimeren von Ianira ab und ist weder eine Ianthopsis noch eine Iolella;

Ianthopsis bolmesi (Richardson) besitzt Epimeren an allen freien Mesomeren (149, S. 466) und dasselbe gilt für Iantbopsis sarsi (Richardson) (149, S. 467); diese Formen gehören deshalb in dieser Hinsicht zu Ianira; die Form des Metsomas aber ist Ianthopsis-ähnlich;

Iolella alascensis Benedict hat Epimeren an allen Mesomeren (149, S. 464);

Iolella glabra Richardson (153, S. 71) zeigt keine Spur von Epimeren.

So könnte man wahrscheinlich weiter gehen. Es ergibt sich aber, dass eine Einteilung in 3 Gattungen bei Ianira nicht durchzuführen ist und ich lasse deshalb die Gattungen Ianthopsis und Iolella fallen, ebenso wie Hansen dies tut (88, S. 14).

Dieselbe Geschichte wiederholt sich gewissermassen bei Iathrippa Bovallius, aber mit Nordenstam (136, S. 172) bin ich der Meinung, dass in diesem Fall die Gattung bestehen bleiben kan.

1 I caudata Richardson.

Anima Sola Island, $13^{\circ} 11^{\prime} 15^{\prime \prime}$ N. $123^{\circ} 02^{\prime} 45^{\prime \prime}$ O., 36 m (158, S. 33$)$.

2 I. japonica Richardson.

Koshika-Inseln, $31^{\circ} 31^{\prime}$ N. $129^{\circ} 25^{\prime} 30^{\prime \prime}$ O., 769 m (150, S. 115).

3. I. chuni (Thielemann).

Fukuura, Sagamibai, $150 \mathrm{~m}$ (207, S. 72).

4. I. abyssicola Beddard.

Kandavu, Fidschi-Inseln, $19^{\circ} 2^{\prime}$ S. $177^{\circ} 10^{\prime}$ O., $2430 \mathrm{~m}$ (14, S. 6).

5. I. neglecta Chilton.

Carnley Harbour, Auckland, $4 \mathrm{~m}$; Port Chalmers and Lyall Bay, Wellington, Neu-Seeland (41, S. 649; 197, S. 355); Neu Amsterdam(?) (218, S. 529).

6 I. monodi Nordenstam.

$10^{\circ}$ S. $80^{\circ} 48^{\prime}$ W., $500 \mathrm{~m}(136$, S. 24$)$.

7 I. occidentalis Walker.

Puget Sound, Washington (149, S. 472).

8 I. triangulata (Richardson).

Monterey Bay, Kalifornien (149, S. 462).

9 I. alascensis (Benedict).

$71^{\circ} 0.2^{\prime}$ N. $157^{\circ} 46^{\prime}$ W., 34 m (149, S. 464); Bering-Meer (77, S. 259; 78, S. 41).

10 I. soldatovi Gurjanova.

Japanisches Meer, daselbst nur im 'Tartaren Sund und Ochotskisches Meer 50—96 m (77, S. 258, 259; 78, S. 42) eine subarktisch-pazifische Form, deren Verbreitung zu den asiatischen subarktischen küsten beschränkt ist (78).

11 I. erostrata (Richardson).

Bering-Meer, Chigagov Harbor, Attu Aleuten (149, S. 465); Japanisches Meer, 65 m, (77. S. 258); Ochotskisches Meer, 56 m, (77, S. 259; 78, S. 41). 
12 I. holmesi (Richardson).

Stephens Passage und Naha Bay, Behm Canal, beide in Alaska, 74-338 m (149, S. 465); BeringMeer, $80-360 \mathrm{~m},(77$, S. 259; 78, S. 42).

13 I. sarsi (Richardson).

Constantine Bay, Amchitka Islands, Alaska, 14 m (149, S. 468); Ochotskisches Meer; Bering-Meer $15-25$ m, (77, S. $259 ; 78$, S. 44).

14 I. angusta Barnard.

Buffel's Bay, False Bay (7, S. 406).

15 I. capensis Barnard.

Sea Point, Kapstadt; St. James, False Bay (4, S: 221).

16 I. exstans Barnard.

Buffel's Bay, Sea Point und Hout Bay, alle Süd-Afrika (6, S. 438).

17 I. bovalli Studer.

Kerguelen (14, S. 11); $47^{\circ} 16^{\prime \prime}$ S., $63^{\circ} 29^{\prime}$ W., 113 m (26, S. 36); Falkland-Inseln und Süd-Georgien, 12-310 m (136, S. 183); Gauss-Station, 385 m (218, S. 544); Victorialand, 450 m (205, S. 200).

Die übrigen 16 Arten sind alle atlantisch oder arktisch; die meisten beschränken sich auf den nördlichen Teil des Atlantischen Ozeans und das Nordpolarmeer; südlicher nur geht exul Müller von Brasilien und minuta Richardson von den Bermuda-Inseln und den Azoren, während capensis Barnard vielleicht auch als atlantisch aufzufassen ist. Überdies sind neglecta Chilton und crosslandi Stebbing mit der atlantischen minıta Richardson verwandt. Was die Tiefen-Verhältnisse betrifft, so sind de Unterschiede sehr gross; maculosa Leach aus dem Nordatlantischen Ozean geht von 9-2052 $\mathrm{m}$, alta von $63-877 \mathrm{~m}$.

\section{Bagatus Nobili}

1 Bagatus stylodactylus Nobili.

Mangarewa; Gambier-Inseln (135, S. 418); Rotes Meer; wahrscheinlich bei Port Sudan, und Golf von Suez. (119, S. 170).

2 Bagatus platydactylus Nobili.

Rikitea; Paumotu-Inseln (?), 2 m (135, S. 418); Mangarewa; Gambier-Inseln (119, S. 170).

3 Bagatus stebbingi Moriod.

Insel Egmont; Scychellen (188, S. 110); Bai von Saint-Georges; Beirut (118, S. 408).

4 Bagatus nanus (Stebbing).

Ceylon; Golf von Manaar (188, S. 50; 119, S. 169).

Es gibt noch eine fünfte Art, B. curvidactylus Nobili; ich habe die dererbezügliche Arbeit (134) leider nicht in den Händen gehabt und der Fundort ist mir unbekannt (119, S. 169).

Von zwei anderen Gattungen kommen keine Arten im Indopazifischen Gebiet vor. Eine von diesen, mit nur einer Art, nl. Austronanus Hodgson ist antarktisch, während Katianira Hansen nord-atlantisch ist. 


\section{Janiropsis G. O. Sars}

1 Janiropsis longiantennata Thielemann. Japan (207, S. 70).

2 Janiropsis serricauda Gurjanova. Japanisches Meer, $42^{\circ} 49^{\prime}$ N., $133^{\circ} 48^{\prime}$ O., $0-2 \mathrm{~m}$ (77, S. 252; 78, S. 46).

3 Janiropsis parva Omer Cooper. Insel Fanning, $3^{\circ} 51^{\prime} 23^{\prime \prime}$ N., $159^{\circ} 21^{\prime} 50^{\prime \prime}$ W. (140 b, S. 4).

4 Janiropsis kincaidi Richardson.

Yakutat; Alaska (149, S. 456); Japanisches Meer; Bering-Meer, 1-3 m, (77 S. 258, 259; 78, S. 45); eine boreal-pazifische Form, die in Zostera-wiesen lebt.

5 Janiropsis derjugini Gurjanova.

Bering-Meer (77, S. 259; 78, S. 46).

6 Janiropsis palpalis Barnard.

Sea Point; Kapstadt; Kalk Bay; False Bay; Süd-Afrika (4, S. 223).

Die siebente Art, J. breviremis G. O. Sars kommt vor bei Bohuslän, Schweden, an der Westküste Norwegens bei Bergen, in der Nordsee und bei West-Irland; Tiefe bis $80 \mathrm{~m}$.

Die Gattung Janiropsis ist der Hauptsache nach eine arktische Gattung.

Jolanthe Beddard

1 Jolanthe acanthonotus Beddard. $65^{\circ} 42^{\prime}$ S., $79^{\circ} 49^{\prime}$ O., $3015 \mathrm{~m}(14$, S. 18).

Die einzige Art.

\section{Acanthaspidea Stebbing}

Die Gattung Acanthaspidea Stebbing umfasst 3 Arten, von welchen typhlops G. O. SARS bei den Lofoten und in der Davisstrasse und bei $65^{\circ} 14^{\prime} \mathrm{N}, 30^{\circ} 39^{\prime} \mathrm{W}, 823-1354 \mathrm{~m}$ vorkommt, decorata Hansen bei 7,5, $21^{\prime}$ W., $4000 \mathrm{~m}$, und dryalskii VANHÖFFEN, Gauss-Station der deutschen Südpolar-Expedition 1901-1903, 350-385 m. Eine Bipolarität fällt nicht zu verkennen, ebenso scheint die Gattung der Tiefsee zu gehören.

\section{Iaerella Richardson}

I. armata Richardson.

Nordwest pazifisch, $52^{\circ} 14^{\prime} 30^{\prime \prime}$ N., $174^{\circ} 13^{\prime}$ O., 540 m (161, S. 635).

Diese Art, sowie Rhactra pulchra von Georges Bank, atlantische Küste Nord-Amerika's aus 3174 m, muss nach HANSEN (88, S. 13), vielleicht mit Recht zu Janira gerechnet werden. Keine anderen Arten dieser Gattungen sind bekannt. 


\section{Iathrippa Bovallius}

1 lathrippa inermis (Haswell).

Port Jackson (91, S. 479).

2 I. sarsi (Pfeffer).

Kerguelen (218, S. 533); Süd-Georgien (141, S. 125); Falkland Inseln (193, S. 354); Süd-Shetlands Inseln und Grahamsland (165, S ); Kap Adare; Victorialand; 81-90 m (205, S. 201); Süd-atlantisch, 100--500 m; Shag Rock Bank, 160 m, (136, S. 177).

\section{3 longicauda (Chilton).}

Golf von Cocovado, Chile (127, S. 135); Lyttelton; Neu-Seeland (26, S. 33), North Cape, Neu-Seeland, 126 m (205, S. 200); Campbell Inseln, Burdwood Bank, Süd-Georgien, 252-310 m; Feuerland, $12-100 \mathrm{~m}$, Magellanstrasse, $180-270 \mathrm{~m}$ (136, S. 176); Ostküste Patagoniens, $48^{\circ} 37^{\prime} \mathrm{S}$., $65^{\circ} 46^{\prime}$ W., $104 \mathrm{~m}$ (157, S. 649); Falkland Inseln, 16-197 m, (136, S. 175).

Die vierte Art $I$. australis (Hodgson) - von TATTERSALl für identisch mit sarsi gehalten - ist antarktisch, Kap Adare (205, S. 201).

\section{Trichopleon Beddard}

1 Trichopleon ramosum Beddard.

Südlich von den Philippinen, 900 m (14, S. 22).

Die einzig bekannte Art.

\section{Pseudoianira Barnard}

1 Pseudoianira stenetrioides Barnard.

Küste Zululandes (9, S. 407).

Die einzig bekannte Art.

\section{Microprotus Richardson}

$1 M$. caecus Richardson.

Bering-Meer, $52^{\circ} 14^{\prime} 30^{\prime \prime}$ N., $174^{\circ} 13^{\prime}$ O., $600 \mathrm{~m}$ (150, S. 118; 78, S. 9). Eine endemische Form des Bering-Meeres.

S. 545$)$.

Die zweite Art, antarcticus Vanhöffen, ist antarktisch aus einer Tiefe von 3398 m (218,

\section{Iaera Leach}

1 laera wakishiana Spence Bate.

Esquimault Harbor, Vancouver, Britisch Kolumbien; 14 m (149, S. 451; 26, S. 49).

2 Iaera pusilla Barnard.

Lion's Head, $171 \mathrm{~m}$ (9, S. 405).

Die übrigen 7 Arten sind alle atlantisch oder mediterran. Hiervon kommt Iaera nordmanni (Rathke) sogar auch im Schwarzen Meer vor. Die früher als Iaera nordmanni (Rathke) für das Kaspische Meer beschriebene Art ist neu und hat von Valkanov den Namen laera sarsi bekommen 
(217). Es soll eine sarmatische Reliktform sein. Iaera sarsi Valkanov wurde im Donau und Theiss sowie im Don angetroffen und wird von Kesselyak (101, S. 240) als Süsswasser-Art betrachtet.

Die 9. Art Iaera sarsi caspica Kesselyak (101, S. 240, Confer auch 217) ist eine eigene Form des Kaspischen Meeres.

In der Gattung Iaera herrschte eine grosse Verwirrung vor. Sie ist neuerdings von verschiedenen Seiten, so von Valkanov (217) und von KesselyaK (101) einer Revision unterzogen worden. Dies hat zu eine bedeutende Umgruppierung der früher zu dieser Gattung gehörigen Formen und Übersiedlung dieser Arten zu anderen Gattungen geführt. Hier wird daher die neueste Einteilung gefolgt. Iaera pusilla jedoch wurde in die Gattung Iaera gelassen. Als Ergebniss der Untersuchungen von MONOD (112) und ARCANGeli (2) stellte es sich heraus, dass die Gattung laera die folgenden 4 Arten umfasst: Iaera albifrons Leach, Iaera nordmanni (Rathke); Iaera hopeana Costa (=I. charieri) und Iaera wakishiana Spence Bate, wozu sich dann die von VAlKanov und Kesselyak neu aufgestellten Arten: Iaera sarsi VALKAnov; Iaera sarsi, caspica Kesselyak; Iaera schellenbergi KESSELYAK, Iaera italica KESELYAK gehören. Inklusiv Iaera pusilla umfasst die Gattung laerd dann insgesamt 9 Arten von denen 7 atlantisch oder mediterran sind.

Nach Kesselyak (102, S. 225) sind die Iaera-Arten Bewohner der nordatlantischen, mediterranen und pontokaspischen Gewässern. Sie leben an beiden Ufern des Nordatlantischen Beckens, an der Nordamerikanischen Küste bis Labrador, an den Küsten Grönlands, der Britischen Inseln und Irland, der Nordsee, der Ostsee, der Azoren und der atlantischen Küsten des europäischen Festlandes. Im Mittelmeer, im Schwarzen Meer, im Kaspischen Meer, teilweise auch in den diesen Salzwassergebieten gehörigen Binnengewässern. In dem südlichen Becken des atlantischen Ozeans, sowie im Antarctis wird Iaera von Neojaera vertreten (136, S. 187). Eine Ausnahme bildet Iaera pusilla, wenn wir diese Art in die Gattung Iaera belassen und nicht auch nach Neojaera überbringen.

\section{Carpias Richardson}

Die Gattung Carpias Richardson mit der einzigen Art C. bermudensis Richardson kommt bei den Bermuda-Inseln vor.

\section{Neojaera Nordenstam}

1 Neojaera serrata (Barnard).

Seapoint bei Kapstadt (6, S. 434).

2 N. antarctica (Pfeffer).

Juan Fernandez (136, S. 189); Kerguelen (218, S. 529); Süd-Georgien (141, S. 58, 134; 101, S. 247); Süd-Atlantisch, 100-500 m; Feuerland, 100 m; Falkland Inseln, 0-22 m; 136, S. 189).

Die einzig bekannten Arten.

Nach Nordenstamm (136) gehört auch Iaera pusilla dieser Gattung zu.

\section{Iais Bovallus}

\section{I. pubescens (Dana).}

Sausalito, Kalifornien (149, S. 455); Insel Campbell (116, S. 11); Ceylon (186, S. 10); Bai von Durban (194, S. 446); Tafelbai; Kapstadt; Saldanha-Bai; False Bay (6, S. 436); Kerguelen (14, 
S. 20; 218, S. 530); Tasmanien (213. S. 15); Okawa; Chatham Islands (47, S. 319); Lyttelton (31, S. 189); Akaroa; Port Chalmers (35, S. 266); Auckland (212, S. 265); Campbell Insel (41, S. 649; 116, S. 111$)$; Dunedin (213, S. 59); Süd-Australien (84, S. 323); Falkland Inseln (195, S. 551; 204, S. 890); Tristan da Cunha (6, S. 436); $51^{\circ} 41^{\prime}$ S. $57^{\circ} 51^{\prime}$ W. (204, S. 890); Kap Hoorn (60, S. 71); Chili; Nassau-Bai; Insel Clarence; Südspitze-Süd Amerika's; Argentinien (116, S. 13); Feuerland (58, S. 744); Staten-Insel (136, S. 179); Süd-Georgien (141, S. 134); Dimamba-Fluss, Kamerun (Süsswasser) und Kap Kamerun (117, S. 1).

Die Varietät longistylis Chilton: Marlborough Sounds und Hawke's Bay; Neu-Seeland und Sydney (42, S. 132); Sud-Australien (84, S. 323).

I hargeri Bovallius.

Magellan-Strasse (26, S. 51).

Beide Formen sind wahrscheinlich identisch, und weil keine anderen Arten von Iais bekannt sind, hat dann die einzige Art eine südlich-circumpoläre universelle Verbreitung.

\section{Ectias Richardson}

Verwandt mit Iais ist die Gattung Ectias Richardson, welche mit der einzigen Art turquete Richardson vorkommt bei Süd-Georgien, den Booth Wandek und Petermann-Inseln, bei Grahamland und Victorialand, 12--90 m (136, S. 187).

\section{Ianirella Bonnier}

\section{Ianirella pusilla Sayce.}

Thorpdale, Victoria, in süssen Wasser (174, S. 125).

Die übrigen 7 Arten der Gattung sind alle atlantisch und nord-atlantisch. Das Vorkommen von pusilla im süssen Wasser in Victoria ist befremdend. Ist diese Form wirklich eine Ianirella? Da mir SAYCE's Beschreibung nicht zur Hand ist, kann ich dies nicht untersuchen.

\section{Protoianira Barnard}

1 Protoianira printicei Barnard.

Kogelberg; Hottentots Holland Mountains; Caledon Division; Süd-Afrika; Süsswasserform (10, S. 167).

Die einzig bekannte Art.

\section{Gruppe Iaeropsini \\ Iaeropsis Koehler}

1 Iaeropsis curvicornis (Nicolet).

Golf von Manaar (188, S. 52). Akaroa, Taylors' Mistake und Lyall Bay, Neu-Seeland (42, S. 133);

Chile (fide 188, S. 52); St. James, False Bay (4, S. 225).

2 Iaeropsis lobata Richardson.

Monterey-Bai, Kalifornien (149, S. 477).

3 Iaeropsis paulensis Vanhöffen.

St. Paul (218, S. 531). 
4. Iaeropsis marionis Beddard.

Insel Marion, $46^{\circ} 43^{\prime}$ S. $38^{\circ} 4^{\prime} 30^{\prime \prime}$ O., 252 m (14, S. 21); Kerguelen (218, S. 531 ).

Die übrigen 5 Arten sind atlantisch oder mediterran, zwei derselben I. patagonicus Richardson und intermedius Nordenstam, beide süd-atlantische Formen, kommen bis zur Südspitze SüdAmerika's vor.

\section{Gruppe Haploniscini}

\section{Haploniscus Richardson}

\section{Haploniscus dimeroceras Barnard.}

Cape Point, 1260 m (7, S. 408).

Von den übrigen 7 Arten sind 5 nord-atlantisch oder arktisch (bicuspis (G. O. Sars), excisus Richardson, retrospinis Richardson, spinifer Hansen und armadilloides Hansen) und 2 (antarcticus Vanhöffen und curvirostris Vanhöffen) antarktisch. Hier liegt also eine bipolare Verbreitung vor.

Hansen.

Die zweite Gattung, Hydroniscus Hansen, umfasst nur den nordatlantischen $H$. abyssi

\section{Gruppe Munnini}

\section{Untergruppe I Antias in i}

Antias Richardson

1 Antias marmoratus Vanhöffen.

Kerguelen; S. Paul (218, S. 534); Süd-Georgien (136, S. 205).

2 Antias bispidus Vanhöffen.

Auckland Insel (197, S. 256); St. Paul (218, S. 533); Grahamland, 95 m; Falkland Insel 25-30 m (136, S. 203).

3 Antias uncinatus Vanhöffen.

Simons Town (218, S. 535).

4 Antias bofsteni Nordenstam.

Cumberland-Bai, Süd-Georgien (136, S. 208).

5 Antias charcoti Richardson.

Inseln Wiencke und Wandel, $20 \mathrm{~m}$ (218, S. 533); Winter Quarters, Mac Murdo Sound, bis $45 \mathrm{~m}$ (98, S. 65). Petermann Insel, Grahamland (151, S. 17; 197, S. 356).

Keine anderen Arten sind bekannt.

\section{Untergruppe II $\mathrm{M}$ u n n in i s. st r. \\ Munna Kröyer}

\section{Munna neo-zelanica Chilton.}

Port Chalmers und Brighton, Neu-Seeland; Auckland Inseln; Campbell Insel (116, S. 14; 36, S. 3); Waikawa Bai, Queen Charlotte Sound bei Picton; Portage, Kenapuru Sound, beide in Neu-Seeland (42, S. 132). 
2 Munna antarctica (Pfeffer).

Kerguelen 2-5 m; Kap. Adare; Winter Quarters (Discovery), Insel Wandel; Wiencke und Petermann 20-70 m (116, S. 14); Süd-Georgien, bis $310 \mathrm{~m}$ (141, S. 58, 137); Victorialand 2-5 m $(98$, S. 58; 218, S. 562).

Die Varetät australis Hodgson kommt allgemein im Antarctis vor, nicht aber in Sud-Georgien $(136$, S. 215).

3 Munna maculata Beddard.

Royal-Sound, Kerguelen, $49^{\circ} 28^{\prime}$ S., $70^{\circ} 13^{\prime}$ O., 50 m (14, S. 26); Observatorybai, Kerguelen (116, S. 20; 218, S. 563) Falkland Inseln, $22 \mathrm{~m}$ (136, S. 211).

4 Munna studeri Hilgendorf.

Kerguelen, 210 m (218, S. 562); Süd-Georgien (116, S. 16).

5 Munna dentata Vanhöffen.

Observatorybai, Kerguelen (218, S. 564).

6 Munna pallida Beddard.

Kerguelen, 55 m (14, S. 27; 116, S. 22); Falkland-Inseln, bis 22 m (136, S. 211).

7 Munna schauinslandi Sars.

Chatham-Inseln (171, S. 372); Kerguelen (218, S. 563).

8 Munna satbneglecta Gurjanova.

Japanisches Meer, 60 m, auch im littoral (77, S. 255; 78, S. 54). Eine endemische Form des Japanischen Meeres (78, S. 244).

9 Munna avatshensis Gurjanova.

Bering-Meer, Ostküste Kamtschatka's, 13-15 m (77, S. 259; 78, S. 53).

10 Munna stephensi Gurjanova.

Bering-Meer, Kommandor Inseln, im littoral (77, S. 259; 78, S. 51).

11 Munna arnboldi Gurjanova.

Bering-Meer, Kommandor Inseln, im littoral (77, S. 259; 78, S. 52).

Auch Miss Richardson meldet das Vorkommen von Munna bei den Pribilov Inseln (148, S. 669). Munna kommt auch vor bei Kad el Hamden, Golf van Suez und bei Jubal (119, S. 172, 173).

Von den übrigen 22 bekannten Arten sind nicht weniger als 10 nord-atlantisch oder nordatlantisch und arktisch, während 4 antarktisch vorkommen und brasiliensis Hansen bei Brasilien. Eine bipolare Verbreitung der Gattung ist also nicht zu verkennen.

\section{Paramunna Sars}

1 Paramunna subtriangulata (Richardson).

Chili; Baie du Torrent; Insel Londonderry; Canal Français; Magellanstrasse, Insel Wiencke (114, S. 16). Süd-Georgien, 1-2 m, Garham Region (136, S. 237), Palmer Archipel (218, S. 572).

2 Paramuna laevifrons Stebbing. $32^{\circ} 53^{\prime} 30^{\prime \prime}$ S., $28^{\circ} 11^{\prime}$ O., $75 \mathrm{~m}(190$, S. 436). 
3 Paramunna concavifrons Barnard.

Durban; Mouille Point bei Kapstadt (7, S. 409).

4 Paramunna capensis Vanhöffen.

Simonstown (218, S. 575).

5 Paramunna kerguelensis Vanhöffen.

Kerguelen (218, S. 574).

6 Paramunna dilatata Vanhöffen.

Observatorybai, Kerguelen (218, S. 573).

7 Paramunna rostrata (Hodgson).

Mac Murdobai, Petermann-Insel; Kerguelen (218, S. 572); antarktisch (98, S. 63); $70^{\circ} 15^{\prime}$ S., $84^{\circ}$ $06^{\prime}$ W. 559 m (114, S. 17); Süd-Georgien 64-74 m; Graham Region (136, S. 239).

8 Paramunna incisa (Richardson).

Insel Wiencke (152, S. 7; 197, S. 358).

9 Paramunna serrata (Richardson).

Palmer Archipel (218, S. 572); Auckland Insein (197, S. 359); Falkland Insein, 1-22 m; Graham Region; Victorialand (136, S. 235).

10 Paramunna gaini (Rìchardson).

Paimer Archipel (218, S. 572); Petermann Insel; Grahamland 1-6 m (152, S. 21; 197, S. 358):

Überdies gibt es noch 4 südliche Arten: integra Nordenstam, von den Falkland Inseln und der Burwood-Bank; dentata Nordenstam von den Falkland Inseln; antarctica Richardson von dem Palmer-Archipel, den Inseln Wiencke und Petermann, von Grahamland und Süd-Georgien, und die antarctische gaussi Vanhöffen und 3 nördliche Arten: bilobata G. O. Sars, brevipes (Bate und Westwood) und typica Tattersall.

\section{Austrosignum Hodgson}

1 Austrosignum glaciale Hodgson.

Maivi Bai, Süd-Georgien (116, S. 12; 136, S. 243); Winter Quarters der National Antarctic Expedition, Tiefe weniger als $36 \mathrm{~m}$ (198, S. 60); Mac-Murdo-Strasse und Gauss-Station der Deutschen Süd-polar-Expedition 1901-1903, 385 m (218, S. 578).

Eine zweite Art: grande Hodgson, ist antarktisch: Winter Quarters (siehe oben, 98, S. 67); eine dritte, falklandicum Nordenstam, wurde bei den Falkland Inseln, 22 m, und der Burwood Bank, 137 - 150 m, gefunden $(136$, S. 245).

Die Gattung Coulmannia Hodgson zählt nur 2 süd-atlantische oder antarktische Arten, nl. frigida Hodgson und australis Hodgson; die mit ihr verwante Gattung notoxenus Hodgson nur die Art spinifer Hodgson, ebenso antarktisch. Dasselbe gilt für Echinomunna Vanhöffen mit der einzigen Art borrida Vanhöffen (218, S. 568).

\section{Pleurocope Walker}

1 Pleurocope dasyura Walker.

Golf von Manaar (188, S. 57), Mittelmeer (220). 


\section{Untergruppe III Pleurogon i i \\ Pleurogonium G. O. Sars}

1 Pleurogonium albidum Beddard.

Christmas Harbour, Kerguelen, 216 m (14, S. 30).

2 Pleurogonium serratum Beddard.

Christmas Harbour, Kerguelen, 216 m (14, S. 31).

Von den übrigen 9 bekannten Arten ist nur eine, minutum Beddard, süd-atlantisch: Tristan da Cunha, 180--270 m; die anderen 6 sind alle nord-atlantisch oder nord-atlantisch und arktisch, sodass diese Gattung wieder als bipolar anzusehen ist.

Verwandt mit Pleurogonium ist Pleurosignum Vanhöffen, mit zwei antarktischen und süd-atlantischen Arten aus 25-385 m, und Antennulosignum Nordenstam mit einer süd-atlantischen Art aus $22 \mathrm{~m}$.

Die vierte Untergruppe der Munnini nach Nordenstam, die Dendrotiinae umfasst die Gattungen Dendrotion G. O. Sars, Mormomunna Vanhöffen, Pseudomunna Hansen und Urias Richardson und fällt ganz ausserhalb unseren Gebietes. Überdies gibt es dann noch verschiedene Gattungen, deren Zugehörigkeit zu den genannten 4 Gruppen Nordenstam's zweifelhaft ist. Ich lasse diese hier folgen.

\section{Astrurus Beddard}

1 Astrutus crucicauda Beddard.

Christmas Harbour, Kerguelen, 216 m (14, S. 39).

Die zweite Art, ornatus Vanhöffen, wurde bei der Gauss-Station der Deutschen SüdpolarExpedition gefangen (218, S. 579).

Neasellus Beddard

1 Neasellus kerguelensis Beddard.

Christmas Harbour, Kerguelen, 216 m (14, S. 35).

Die einzig bekannte Art. Die Stellung dieser Gattung ist zweifelhaft (136, S. 198).

\section{Acanthomunna Beddard}

1 Acantbomunna proteus Beddard.

Neu-Seeland, $40^{\circ} 28^{\prime}$ S., $177^{\circ} 43^{\prime}$ O., $1980 \mathrm{~m}$ und $37^{\circ} 34^{\prime}$ S., $179^{\circ} 22^{\prime}$ O., $1260 \mathrm{~m}$ (14, S. 50 ).

Die einzig bekannte Art.

Diese Gattung, sowie Neasellus wird bei den Munnini gestellt, obwohl Nordenstam mit Recht behauptet, dass ihre richtige Stellung im System nicht angegeben werden kan (136, S. 198).

\section{Kuphomunna Barnard}

1 Kuphomunna rostrata Barnard. . Buffels Bay, False Bay (6, S. 440; 7, S. 410).

Die einzig bekannte Art. 


\section{Haplomunna Richardson}

1 Haplomunna coeca (Richardson).

Santa Catalina-Inseln, Süd-Kalifornien, $33^{\circ} 2^{\prime} 15^{\prime \prime}$ N. $120^{\circ} 42^{\prime}$ W, 3928 m (149, S. 485).

Die einzig bekannte Art.

Die Gattung Caecimunna Richardson umfasst nur eine einzige Art, truncata Richardson, welche bei Martha's Vineyard, $702 \mathrm{~m}$, und bei Halifax, $144 \mathrm{~m}$, erbeutet wurde (153, S. 80).

Munnella Bonnier mit der Art danteci Bonnier lebt im Bai von Gascogne in einer Tiefe von $950 \mathrm{~m}$ (19, S. 593). Echinomunna horrida Vanhöffen ist antarktisch, $358 \mathrm{~m}$ (218, S. 568).

\section{Gruppe Ischnomesini}

\section{Heteromesus Richardson}

\section{Heteromesus thomsoni (Beddard).}

Nord-pazifisch, $36^{\circ} 10^{\prime}$ N., $178^{\circ}$ O., 3690 m (14, S. 172).

Die Gattung ist übrigens atlantisch und zwar kommt sie nur im nördlichen Teil vor; am meisten südlich gehen spinosus (Beddard) von den Açoren, $37^{\circ} 26^{\prime} \mathrm{N} ., 25^{\circ} 13^{\prime} \mathrm{W} .1800 \mathrm{~m}$, und similis Richardson, ebenso von den Açoren (S. Miguel), 2995 m. Die übrigen 7 gehen nördlicher, sogar bis in das arktische Gebiet.

\section{Rhabdomesus Richardson}

1 Rbabdomesus bacillus (Beddard).

S. W. von Melbourne, $50^{\circ}, 1^{\prime}$ S., $123^{\circ} 4^{\prime}$ O., $3240 \mathrm{~m}$ (14, S. 44).

2 Rhabdomesus bacilloides (Beddard).

S. W. von Valparaiso, 42 ${ }^{\circ} 43^{\prime}$ S., $82^{\circ} 11^{\prime}$ W., 2610 m (14, S. 47).

3 Rbabdomesus bacillopsis Barnard.

Cape Point, 1260 m (7, S. 414).

Die vierte Art inermis Vanhöffen ist antarktisch, 2450 m. Nach HANSEN (88, S. 56) wäre es besser diese 4 Arten zu Ischnomesus zu rechnen.

Es gibt noch die Gattungen Ischnomesus mit 3 nord-atlantischen Arten und Haplomesus mit 5 nord-atlantischen und arktischen Arten aus der Tiefsee.

\section{Gruppe Macrostylini}

\section{Macrostylis G. O. Sars}

1 Macrostylis latifrons Beddard.

Nord-pazifisch, $36^{\circ} 10^{\prime}$ N., $178^{\circ}$ O., 3690 m (14, S. 175).

2 Macrostylis spiniceps Barnard.

Cape Point, $1260 \mathrm{~m}$ (7, S. 412).

Die 6 übrigen Arten sind alle nord-atlantisch oder arktisch aus grösseren Tiefen. 


\section{Gruppe Nannoniscini}

\section{Austrofilius Hodgson}

\section{Austrofilius furcatus Hodgson.}

Observatory, Kerguelen, $10 \mathrm{~m}$; Simonsbai, Kapland (218. S. 554); Mac Murdosund, weniger als 36 m (98, S. 52); Grahamland, 125 m; Süd-Georgien, 22 m; Falkland-Inseln, 22 m; Feuerland, $35 \mathrm{~m}(136$, S. 253).

Die beiden anderen Arten, serratus Vanhöffen und octodentatus Vanhöffen sind antarktisch.

\section{Austroniscus Vanhöffen}

1 Austroniscus ectiformis Vanhöffen.

Observatorybai, Kerguelen (218, S. 553).

Die beiden anderen Arten, ovalis Vanhöffen und rotındatus Vanhöffen sind antarktisch, 70 und $385 \mathrm{~m}(218$, S. 551, 552).

Von den anderen drei Gattungen der Gruppen kommen keine Arten im Indo-pazifischen Gebiet vor. Nannoniscus G. O. Sars zählt 17 Arten, von denen 2 antarktisch sind: australis Vanhöffen und bidens Vanhöffen; eine findet man im Kaspischen Meer: caspius G. O. Sars; die übrigen sind nordatlantisch und arktisch. Die einzige Art von Nannoniscoides Hansen, N. angulatus Hansen ist nord-atlantisch und arktisch. Die ganze Gruppe ist also ausgesprochen bipolar.

\section{Gruppe Desmosomatini \\ Desmosoma G. O. Sars}

$\mathrm{Zu}$ dieser Gattung gehören etwa 24 Arten (confer Hansen (88) und HulT (99A) S. 111; 99B, S. 19), von denen die meisten nord-atlantisch, bis im Mittelmeer, oder arktisch sind. Antarktisch ist nur longimanus (Vanhöffen) (218, S. 559), während MONOD eine Art von $70^{\circ} 15^{\prime} \mathrm{S}, 84^{\circ} 06^{\prime}$ W, aus 569 m vermeldet $(114$, S. 15). Überdies hat NoRDENSTAM 4 Arten von Süd-Georgien und den Falkland Inseln $(218$, S. 254, 264) und 4 süd-atlantische Formen beschrieben. Dennoch muss ich sie erwähnen, da BARnARD (7, S. 411) das Vorkommen von 2 mutilierten Exemplaren von Cape Point, 1260 m vermeldet, welche er zu Eugerda Meinert rechnen will. Er macht dabei aber einen gewissen Vorbehalt. Ist aber diese Zugehörigkeit zu Eıtgerda richtig, dann haben wir es mit einer DesmosomaArt zu tun, da HANSEN (88, S. 107) ausdrücklich und ganz zurecht betont, dass die Eugerda-Arten zur Gattung Desmosoma gerechnet werden müssen.

\section{Ilychthonos Barnard}

\section{Ilychthonos capensis Barnard.} Cape Point, 1260 m (7, S. 416).

Die einzig bekannte Art.

Die dritte Gattung, Echinopleura G. O. Sars, ist nord-atlantisch, die vierte, Dactylostylis Richardson, atlantisch. 


\section{Gruppe Ilyarachnin}

Ilyarachna G. O. Sars

1 Ilyarachna starkadomsk.ii Gurjanova.

Ochotskisches Meer, Japanisches Meer 100-591 m (77, S. 258; 78, S. 57).

Eine subarktische-pazifisch-asiatische Form, die sich an dem Ausstausch der Faunen der genannten Meeren beteiligt (78).

2 Ilyarachna zachsi Gurjanova.

Japanisches Meer, 105—780 m (77, S. 258; 78, S. 36).

3 Ilyarachna affinis Barnard.

Cape Point, 1260 m (7, S. 419).

4 Ilyarachna crassiceps Barnard.

Cape Point, $1260 \mathrm{~m}$ (7, S. 420).

Die übrigen 11 bekannten Arten sind atlantisch oder arktisch; am südlichsten gehen $I$. abyssorum Richardson von den Azoren an einer Tiefe von 4060-4165 m (159 S., 534), und I. antarctica Vanhöffen von der Gauss-Station der Deutschen Südpolar-Expedition aus 3397-3423 m (218, S. 591), und Sud-Georgien, 252-310 m (136, S. 266).

\section{Echinozone G. O. Sars}

1 Echinozone quadrispinosa (Beddard).

Cumbulandbai, Kerguelen, $48^{\circ} 45^{\prime}$ S., $69^{\circ} 14^{\prime}$ O., 229 m (14, S. 78); Graham Region, 360 m?; Süd-Georgien, 22--310 m; Victoria-Land (136, S. 273); $71^{\circ} 19^{\prime}$ S., $87^{\circ} 37^{\prime} \mathrm{W}$., $400 \mathrm{~m} ; 71^{\circ} 14^{\prime}$ S., $89^{\circ} 14^{\prime}$ W., $400 \mathrm{~m}$ und $70^{\circ} 15^{\prime}$ S., $84^{\circ} 06^{\prime}$ W., $569 \mathrm{~m}$ (114, S. 23), 'Tiefe 200-1500 m.

Es gibt noch 4 Arten, von denen 2, coronata (G. O. Sars) und arctica Hansen, aus dem Nord-atlantischen und arktischen Gebiet stammen, während die dritte, magnifica Vanhöffen, antarktisch ist.

Die anderen Gattungen der Gruppen: Aspidarachna G. O. Sars und Pseudarachna G. O. Sars sind nicht indo-pazifisch.

Von den beiden Arten von Aspidarachne G. O. Sars ist clypeata G. O. Sars bei den Lofoten und an der Finmarkischen Küste, 216-450 m gefunden worden, während aries Vanhöffen bei der genannten Gauss-Station in $385 \mathrm{~m}$ vorkommt. Dasselbe gilt für die beiden Arten von Pseudarachne: birsuta G. O. Sars ist norwegisch, $54 \mathrm{~m}$, spicata (Hodgson) antarktisch, bis $385 \mathrm{~m}$. Die bipolare Verbreitung ist also deutlich.

\section{Gruppe Eurycopini}

\section{Eurycope G. O. Sars}

1 Eurycope pellucida Beddard. Neu-Guinea, $2^{\circ} 33^{\prime}$ S., $144^{\circ} 4^{\prime}$ O., 1926 m (14, S. 74).

2 Eurycope laevis Richardson.

Koshika-Inseln, $31^{\circ} 39^{\prime} 30^{\prime \prime}$ N., $129^{\circ} 24^{\prime}$ O., 751 m; Bering-Meer, $54^{\circ} 33^{\prime}$ N., $178^{\circ} 45^{\prime}$ O., $1100 \mathrm{~m}$; 
Otchishi Saki, Hokkaido, Pazifisch $2^{\circ} 02^{\prime} 40^{\prime \prime}$ N., $142^{\circ} 33^{\prime} 20^{\prime \prime}$ O., 628 m (155, S. 120). Japanisches Meer, Tiefe 680-1100 m (78, S. 61).

Wird von Gurjanova zur Gattung Munnopsurtes gebracht (78, S. 60). Es ist diese Art eine Tiefseeform des Stillen Ozeans.

3 Eurycope spinifrons Gurjanova.

Japanisches Meer 308-3000 m (77, S. 258; 78, S. 63).

4 Eurycope pavlenko: (Gurjanova).

Japanisches Meer, Tiefe bis 60 m, (77, S. 258; 78, S. 63).

5 Eurycope scabra Hansen.

$2^{\circ} 34^{\prime}$ N., $92^{\circ} 6^{\prime}$ W., $2448 \mathrm{~m}(86$, S. 101$) ; 2^{\circ} 34^{\prime}$ N., $92^{\circ} 6^{\prime}$ W., $2448 \mathrm{~m}(219$, S. 184$)$.

6 Eurycope sulcifrons Barnard.

Cape Point, $1260 \mathrm{~m} \mathrm{(7,} \mathrm{S.} \mathrm{422).}$

7 Eurycope quadrata Barnard.

Cape Point, $1260 \mathrm{~m}$ (7, S. 422).

8 Eurycope fusiformis Barnard.

Cape Point, $1260 \mathrm{~m}$ (7, S. 423).

9 Eurycope sarsi Beddard.

Inseln Marion, $46^{\circ} 16^{\prime}$ S., $48^{\circ} 27^{\prime}$ O., 2880 m (14, S. 61).

10 Eurycope murrayi (Walker).

Bai von Bengalen, $9^{\circ} 8^{\prime}$ N., $87^{\circ} 25^{\prime}$ O. (12, S. 188$)$.

Gebiet von Kap Verdun und von diesen Kap zu den Marion Inseln (218, S. 581). Nordatlantisch bis der Strasse von Gibraltar, 1130-4000 m, (198, S. 11; 88, S. 139).

11 Eurycope spinosa Beddard.

$53^{\circ} 55^{\prime}$ S., $108^{\circ} 35^{\prime}$ O., $3510 \mathrm{~m}(14$, S. 69$)$.

Es sind überdies noch 24 anderen Arten von Eurycope beschrieben worden. Von diesen sind 6 antarktisch, die übrigen sind alle atlantisch und zwar grösstenteils nord-atlantisch, und arktisch; am südlichsten gehen abyssicola Beddard von den Açoren, $3915 \mathrm{~m}$, nobilii, Richardson, atlantica Bonnier, beddardi Bonnier, complanata Bonnier und parva Bonnier, alle aus dem Golf von Biscaye, aus Tiefen von $950-11.07 \mathrm{~m}$.

\section{Storthyngura Vanhöffen}

1 Storthyngura intermedia (Beddard).

Sandwich-Inseln, $37^{\circ} 52^{\prime}$ N., $160^{\circ} 17^{\prime}$ W., 4932 m (14, S. 71).

2 Storthyngura pulchra (Hansen).

$6^{\circ} 10^{\prime}$ N., $83^{\circ} 6^{\prime}$ W.; $2648 \mathrm{~m} ; 2^{\circ} 34^{\prime}$ N., 92" $6^{\prime}$ W., $2448 \mathrm{~m}(86$, S. 100$) ; 2^{\circ} 34^{\prime}$ N., $92^{\circ} 6^{\prime}$ W., $2448-2648 \mathrm{~m}(24$, S. 184).

3 Stortbyngura fragilis (Beddard).

Yokohama, $34^{\circ} 37^{\prime}$ N., $140^{\circ} 32^{\prime}$ O., $3375 \mathrm{~m}$; Insel Marion, $46^{\circ} 16^{\prime}$ S., $48^{\circ} 27^{\prime}$ O., $2880 \mathrm{~m} ; 60^{\circ} 52^{\prime}$ S., $80^{\circ} 20^{\prime \prime}$ O., $2268 \mathrm{~m}$; $50^{\circ} 1^{\prime}$ S., $123^{\circ} 4^{\prime}$ O., $3240 \mathrm{~m}$ (14, S. 66).

4 Storthyngura novae-zeelandiae (Beddard).

Neu-Seeland, $40^{\circ} 28^{\prime}$ S., $177^{\circ} 43^{\prime}$ O., $1980 \mathrm{~m}$ (14, S. 63). 
Die übrigen 6 Arten kommen an verschiedenen Stellen vor; St. elegans Vanhöffen ist antarktisch, 400-3423 m; caribbea (Richardson) findet man in West-Indien, $1237 \mathrm{~m}$; truncata (Richardson) an der Ostküste Nord-Amerika's, 2745-3184 m; atlantica (Beddard) bei den Azoren, $38^{\circ} 11^{\prime}$ N. $27^{\circ} 9^{\prime}$ W., $162 \mathrm{~m}$; magnispinis (Richardson) bei Nantucket Shoals, $2641 \mathrm{~m}$; und robustissima Monod $71^{\circ} 14^{\prime}$ S. $89^{\circ} 14^{\prime}$ W., $400 \mathrm{~m}$.

\section{Munnopsurus Richardson}

1 Munnopsurus giganteus (G. O. Sars).

Bering-Meer, $13 \mathrm{~m}$; Ochotskisches Meer, 13-1645 m (77, S. 259; 78, S. 59); Nord-atlantisch und Arktisch, Grönland, 30-1404 m (88, S. 135); Ost-Grönland, 78--80 m (137, S. 3); Baffin Land, $75-930 \mathrm{~m}(198$, S. 11).

Die Art soll arktisch, vermutlich circumpolar sein. Die von GurJanova aufgestellte Varietät (78, S. 59) ochotensis Gurjanova wurde nur im Ochotskischen Meer angetroffen in Tiefen von $80-645 \mathrm{~m}$. Das Vorkommen dieser Form im Bering-Meer weist auf dem Eindringen der arktischen Formen in diese Gewässer hin. Sie dringt aus dem Eismeer in dem Stillen Ozean durch (78).

2 Munnopsurus longipes (Tattersall).

Lion's Head und Cape Point, Süd-Afrika, 434-1260 m (5, S. 227); westlich von Irland, 630-817 m; $61^{\circ} 15^{\prime}$ N. $9^{\circ} 35^{\prime}$ W., 833-927 m; Davis-Strasse, 2583 m (88, S. 136).

3 Munnopsurus minutus Gurjanova.

Japanisches Meer, 190—440 m (77, S. 258; 78, S. 62).

Es gibt weiter noch $M$. australis (Vanhöffen) aus dem Südpolarmeer, 400-3423 m. Die vierte Gattung, Syneurycope Hansen, mit der einzigen Art S. parallela Hansen, ist nord-atlantisch, $58^{\circ} 10^{\prime}$ N., $48^{\circ} 25^{\prime}$ W., $3321 \mathrm{~m}$.

\section{Gruppe Munnopsini}

\section{Munnopsis M. Sars}

1 Munnopsis latifrons Beddard.

Insel Ino Sima, Japan, $35^{\circ} 11^{\prime}$ N., $139^{\circ} 28^{\prime}$ O., $621 \mathrm{~m}$ (14, S. 57); Omai Saki Light, $34^{\circ} 5^{\prime} \mathrm{N}$., $137^{\circ} 59^{\prime}$ O., und Kusakaki Jima, $30^{\circ} 34^{\prime}$ N., $129^{\circ} 19^{\prime} 30^{\prime \prime}$ O.; $792--1192$ m (155, S. 119). (confer 78, S. 65).

2 Munnopsis gracilis Beddard.

Neu-Seeland, $40^{\circ} 28^{\prime}$ S., $177^{\circ} 43^{\prime}$ O., $1980 \mathrm{~m}$ (14, S. 52).

3 Munnopsis longiremis Richardson.

Galapagos-Inseln, 1462 m (163, S. 162); Chatham-Insel, 1462 m (219, S. 184).

Noch an anderen Stellen des nordwestlichen Teiles des pazifischen Ozeans kommen Formen vor, welche aber nicht näher beschrieben worden sind (155, S. 119); ebenso bei Neu-Seeland, zwischen Neu-Guinea und Japan, Kerguelen, den Crozet-Inseln und den Açoren. Der Typus, M. typica M. Sars, ist nord-atlantisch und arktisch, circumpolar, von Island bis Ost-Grönland (137, S. 3), Tiefe 43-275 m. Confer auch StePhensen (199, S. 6) 70-326 m, eine eurybathybische Art, grösste Tiefe bis $800 \mathrm{~m}$ und Stephensen (198, S. 12) Baffins Land, Tiefe bis $200 \mathrm{~m}$. 


\section{Pseudomunnopsis Hansen}

1 Pseudomunnopsis beddardi (Tattersall).

Cape Point, $1260 \mathrm{~m}$ (7, S. 418); weiter Westküste Irlands, 358-688 m; Faroer, $61^{\circ} 15^{\prime}$ N., $9^{\circ} 35^{\prime}$ W., 833-927 m; Davis-Strasse, $61^{\circ} 50^{\prime}$ N., 56 $21^{\prime}$ W., $2583 \mathrm{~m}$ (88, S. 161).

Die einzig bekannte Art.

\section{Munnopsoides 'Tattersall}

1 Munnopsoides australis (Beddard).

Insel Marion, $46^{\circ} 16^{\prime}$ S., $48^{\circ} 27^{\prime}$ O., $2880 \mathrm{~m}$ (14, S. 56); $78^{\circ}$ S., $88^{\circ} 48^{\prime}$ W., und $71^{\circ} 19^{\prime}$ S., $87^{\circ}$ $37^{\prime} \mathrm{W}$; $400-500 \mathrm{~m}(114$, S. 25).

Die zweite Art, exmius Hansen ist nord-atlantisch, 833-2583 m; die Verbreitung ist deshalb wieder bipolar. Die nahe verwandte Pseudomunnopsis beddardi (Tattersall) (einzige Art der Gattung) ist nord-atlantisch, 358-2583 m.

Die Gattung Paramunnopsis Hansen umfasst 5 Arten, von denen zwei mittel-atlantisch sind: longicornis (Hansen) von Fernando Noronha, 3.6 $6^{\circ}$ S. $33.2^{\circ} \mathrm{W} ., 600-800 \mathrm{~m}$, und spinifera (Vanhöffen) bei Ascension und den Kap Verdischen Inseln, 400-3000 m; die dritte Art oceanica (Tattersall) ist atlantisch, 0-3000 m (198, S. 12).

Die einzige Art der Gattung Lipomera Tattersall ist nord-atlantisch.

\section{Acanthocope Beddard}

1 Acanthocope acutuspina Beddard.

S. W. von Valparaiso, $42^{\circ} 43^{\prime}$ S., $82^{\circ} 11^{\prime}$ W., $2610 \mathrm{~m}$ (14, S. 85).

2 Acanthocope spinicauda Beddard. $52^{\circ} 1^{\prime}$ S., $123^{\circ} 4^{\prime}$ O., $3240 \mathrm{~m}(14$, S. 82$)$

Keine anderen Arten sind bekannt.

Die übrigen Gruppen: Thambematini, Pseudomesini und Schistosomatini, jede mit nur eine Art, sind alle nord-atlantisch.

\section{Unterordnung PHREATOICIDEA}

Man vergleiche für die Verwandtschaftsverhältnissen: 10, S. 161; 122, S. 179.

\section{Phreatoicus Chilton}

1 Phreatoicus australis Chilton.

Mount Kosciusko, Neu-Süd-Wales, 5700 Fuss hoch; Mount Wellington, Tasmanien, 4100 F'uss hoch; Huntingfields und in einer Lagune auf der Insel Bruni, Meeresspiegel höhe; Mount Baw Baw, Victoria, Süsswasser (177, S. 109; 10, S. 158, 144).

2 Pbreatoicus shephardi Sayce.

Wallaly Creek, Plenty Ranges, Victoria, 2000 Fuss (177, S. 112); Manning-Fluss, Neu-Süd-Wales, 5000 Fuss (10, S. 159; 43, S. 91). 
3 Pbreatoicus tasmaniae Thomson.

Great Lake von Tasmanien, 3880 Fuss hoch (177, S. 99; 5, S. 231).

4 Pbreatoicus brevicaudatus Smith.

Great Lake von Tasmanien; Swan Bay und Mc. Lanagan’s Bay vom Great Lake (177, S. 102; 10, S. 159).

5 Phreatoicus tattersalli Sheppard.

Todd's Corner, Great Lake von Tasmanien (177, S. 105).

6 Phreatoicus chiltoni Sheppard.

Great Lake von Tasmanien (177, S. 108).

7 Pbreatoicus kirki Chilton.

Insel Ruapuke, Neu-Seeland, Süsswasser (39, S. 275).

Die Varietät dunedensis Chilton in Süsswasser bei Mosgiel und Woodhaugh bei Dunedin (177, S. $111 ; 10$, S. 160).

8 Phreatoicus joyneri Nichols.

Tasmanien (122, S. 183).

9 Pbreatoicus assimilis Chilton.

In Brunnen bei Winchester, Canterbury, Neu-Seeland (37, S. 186; 10, S. 159).

10 Phreatoicus typicus Chilton.

Eyreton, Neu Seeland; Ashburton, Süsswasser (177, S. 110; 37, S. 196; 10, S. 159).

11 Pbreatoicus capensis Barnard.

Auf der Spitze von Tafelberg, 3000 Fuss (5, S. 233).

Die Varietät abbreviatus Barnard: Kogelberg, Hottentots Holland Mountains (10, S. 157).

Die Varietät depressus Barnard: Forest Ranger's Hut in Steenbras Valley, Hottentots Holland Mountains (10, S. 157).

Es sind keine anderen Arten bekannt, sodass die Gattung sich auf kleines Gebiet beschränkt. Merkwürdig ist die Tatsache, dass Phreatoicus schon im Trias von Neu-Süd-Wales gefunden worden ist (Phr. wianamattensis Chilton); er ist also eine sehr alte Form (44).

\section{Phraeatomerus Sheppard}

1 Pbraeatomerus latipes (Chilton).

Maree (Hergott), in einer Süsswasserbrunnen; Coward, Central Australien, Süsswasser (177, S. 115).

2 Phraeatomerus palustris (Glauert).

Chinaman's Garden, Smith's Lake, North Perth; Balcatta Beach bei Perth, West-Australien (177, S. 116).

Keine anderen Arten sind bekannt. 


\section{Phraetoicopsis Spencer et Hall}

1 Pbraeatoicopsis terricola Spencer et Hall.

Gellibrand River bei Colac, Victoria; Otway Forest; Fort William bei Ararat; the Grampians, Victoria $(177$, S. 118). (Confer 10, S. 142, 160).

Die einzig bekannte Art.

\section{Amphiscopus Nicholls}

1. Amphiscopus lintoni (Nicholls).

Mund von King River, West Australien, Süsswasser (122, S. 117).

2 Ampbiscopus ambigutus Sheard.

The Grampians, Wasserfälle, Victoria, Süsswasser (176, S. 473).

\section{Phreatoicoides Barnard}

1 Pbreatoicoides plumosus (Nicholls et Milner).

Lesmurdie Falls, Darling Range, West-Australien, Süsswasser (177, S. 120).

2 Phreatoicoides gracilis Sayce.

Narracan-Fluss, Thorpdale, Gippsland, Victoria (177, S. 121; 5, S. 232).

Keine anderen Arten sind bekannt.

\section{Eophreatoicus Nicholls}

1 Eopbreatoicus kershawi Nicholls.

Northern Tenritory, Australien (122, S. 191).

Die einzig bekannte Art.

\section{Hypsimetopus Sayce}

1 Hypsimetopus intrusor Sayce.

Zeehan, Tasmanien (177, S. 123; 5, S. 232).

Die einzig bekannte Art.

Die Verbreitung dieser sehr alten Familie ist interessant: Australien, Tasmanien und NeuSeeland einerseits, Süd-Afrika anderseits. Es unterliegt keinem Zweifel, dass die Tiere dieser zwei Gebiete miteinander verwandt sind; BARNARD weist mit Nachdruck auf die Verwandtschaft zwischen Pbreatoicus capensis mit australis hin (10, S. 159). Man kann wohl mit Sicherheit behaupten, dass die Pbreatoicidae noch an vielen anderen Stellen im indo-pazifischen Gebiet vorkommen; man hat aber niemals nach diesen Formen gesucht. 


\section{SCHRIFTTUM}

11925 Arcangeli, A. Notizie supra alcuni Isopodi del mare mediterraneo, Atti, Soc. Ital. Sci. Nat. Milano 63: 312-319.

21934 - Il Genere Jaera LEACH nel mediterraneo a la convivenza occasionale die Jaera hopeana Costa con Sphaeroma serratum (Fabr.) Boll. Mus. Zool. Torino 44, (3) : 273-292.

31913 Barnard, K. H. Phreatoicus in South Africa, Nature 91: 372.

41914 - Contributions to the Crustacean Fauna of South Africa 1. Additions to the marine Isopoda, Ann. South African Mus. 10: 197-242.

51914 - Contribution to the Crustacean Fauna of South Africa 2. Description of a new species of Phreatoicus (Isopoda) from S. Africa; Ann. S. Afr. Museum 10: 231-240.

61914 - Contributions to the Crustacean Fauna of South Africa 3. Additions to the marine Isopoda, with notes on some previously incompletely known species, Ann. S. African Museum 10: 325a358 a, 359-442.

71920 Contributions to the Crustacean Fauna of South Africa No. 6. Further additions to the list of marine Isopoda, Annals Souh African Mus. 17, Part 5: 319-438.

81925 A revision of the family Antburidae (Crustacea Isopoda), with Remarks on certain morphological peculiarities, Jl. Linn. Soc. 36: 109-160.

91925 Contributions to the Crustacean Fauna of South Africa No. 9. Further additions to the List of Isopoda, Ann. South African Mus. Vol. 20: 381-412.

101927 A study of the freshwater Isopoda and Amphipodan Crustacea of South Africa, Trans Roy, Soc. South Africa 14: 139-215.

111935 Report on some Amphipoda, Isopoda and Tanaidacea in the Collections of the Indian Museum, Rec. Ind. Mus. 37: 279-319.

121936 Isopods collected by the R.I.M.S. „Investigator”, Rec. Ind. Mus. 38: 147-191.

131885 BedDard, A. Preliminary notice of the Isopoda collected during the Voyage of H. M. S. Challenger, Pt. II Munnopsidae; Proc. Zool. Soc. London.

14. 1886 Report on the Isopoda collected by H. M .S. Challenger during the years 1873-1876 Part II, Challenger Reports, Zool. 17: 1-178.

151897 Benedict, J. E. A revision of the Genus Synidotea, Proc. Acad. Nat. Sc. Philadelphia: 389—404.

161898 - The Arcturidae in the U.S. National Mus., Proc. Biol. Soc. Washington 12: 41-51.

171898 Two new Isopods of the Genus Idotea from Coast of California, Proc. Biol. Soc. Washingtn 12.

181934 BERG, L. C. Über amphiboreale (disjunkte) Verbreiterung der Meeresfauna auf der nördlichen Halbkugel, Zoogeographica II, 3.

191896 Bonnier, J. Edriophthalmes, Résultats scientifiques Campagne „Candan” Golfe du Gascogne; Ann. Univ. Lyon 1896: 527---689.

201918 Boone, P. L. Descriptions of ten new Isopods. Proc. Stat. National Mus. 54: 591-604.

211920 — Isopoda, Reprt, Canadian Arctic Exped., 1913-1918 Vol. VII Crustacea pt. D. Ottawa: 1—40.

221923 New marine Tanaid and Isopod Crustacea from California, Proc. Biol. Soc. Washington 36: $147-156$.

$231927-$ Crustacea frm tropical east American Seas, Buil. Bingham Ocean Coll. New York I; 2: 1-147.

24. 1910 Bouvier, E. L. Quelques Crustacés de l'Amerique et des Sandwich du Sud, Revista chilena 14: $179-182$.

251911 Notes sur les Arcturus, Annales del Museo nacional de Historia natural de Buenos Aires (3) 14: 401.

261886 Bovallius, C. Notes on the family Asellidae, Bihang K. Svenska Vet. Akad. Hand. 11, No. 15: 1-54.

271909 Calman, W. T. A Treatise on Zoology by E. Ray Lankaster, Vol. 7 Appendiculata, Teil 3.

281878 Chilton, Ch. Additions to the New Zealand Crustacea, Transact. New Zealand Crustacea Transact. New Zealand Institute 11: 171-174.

291881 On some subterranean Crustacea, Transact. N. Zealand Institute 14: 174-180.

301883 Further additions to our knowledge of the New Zealand Crustacea, Trans N.Z., Inst. XV 1882: 72 . 73 . 
$31 \quad 1883$

$32 \quad 1883$

331890

34. 1891

$35 \quad 1892$

$36 \quad 1892$

$37 \quad 1894$

381905

391905

$40 \quad 1906$

$41 \quad 1909$

511916

1917

Chilton, CH. In two new Isopods, Transact. Proc. New Zealand Inst. 15, 1882: 188-190.

- Addition to the Crustacea of New Zealand, Transact. Proc. New Zealand Institute 16: 249

- Revision of the New Zealand Idoteidae, Transact. New Zealand Inst. 22: 189-204.

- Idotea lacustris G. M. THomson, New Zealand Jl. Sc. n.s. I: 131-132.

- Notes on some New Zealand Amphipoda and Isopoda, Transact. New Zealand Instit. 1891

24: $258-269$.

- A new species of Munna from New Zealand, Ann. Mag. Nat. Hist. (6) 9: 1-12.

- The subterranean Crustacea of New Zealand, Trans. Linn. Soc. London (2) 6: 163-284.

- List of Crustacea from the Chatham Islands, Transact, New Zealand Instit. 38: 269-273.

- Description of a species of Phreatoicus from the surface waters of New Zealand; Transact.

Proc. New Zealand Inst. 38: 274-275.

Notes on some Crustacea from the fresh water lakes of New Sealand, Proc. Z. Soc. London: $702-705$. New Zealand, Repts. 2: 601-671.

1912 - Miscellaneous notes on some New Zealand Crustacea, Transact, N. Zeal. Inst. 44: 128-135.

1916 Some Amphipoda and Isopoda from Barrington Tops (4600 Feet altit.) N. South Wales, Jl.

Roy Soc. N.S. Wales, Sydney 50: 82-98.

1918 A fossil Isopod belonging to the Freshwater Genus Phreatoicus, Pr. Roy, Soc. N.S.W. 51: $365-388$.

1922 The flora and fauna of Nuyt's Archipelago and the Investigator group No. 1 Amphipoda and Isopoda, Proc. R. Soc. S. Australia 46: 34-38.

1924 - Fauna of the Chilka Lake, Tanaidacea and Isopoda, Mem. Ind. Mus. Calcutta 5: 875—895.

1925 Some amphipoda and isopoda from the Chatham Islands, Rec. Canterbury Mus. 2: 317-320.

1915 Collinge, W. E. Some observations on the Isopod Idotea hectica (Pallas), Ann. Mag. Natur. Hist.

(8) 16: 161-164.

1916 On the structure of the marine Isopod Mesidotea sibirica (Birula) with some remarks upon allied Genera, Jl. Zool. Res. 1916 I: 112-118.

1916 Some remarks upon the structure and generic position of Idotea lacustris Thomson, Jl. Zool. Res. 1: 153-157.

Research, London 1: 33-35.

1916 On the marine Isopod Idotea ochotensis BrandT, Jl. of Zool. Res. 1: 82-85.

1917 - A revision of the British Idoteidae, a family of marine Isopoda, Transact, R. Soc. Edinburgh 51: $721-760$.

1917 - Description of a new species of Isopoda of the Genus Paridotea, Stebbing., Jl. Zool. Res. II, Pt. 3: $111-114$.

Description of a new species of Isopoda of the Genus Synidotea HARGER, from the Gulf of Manaar, Rec Ind. Mus. Calcutta 13: 1-3.

1918 - On the oral appendages of certain species of marine Isopoda, J1. Linn. Soc. Zool. 34: 65—93. 1918 Some observations on two rare marine isopods, Jl. Zool. Res. 3: 63-79.

1853 Dana, J. D. Crustacea, Pt. 1, United States exploring Exped. 1838-1842, 13: 696-806.

1870 Dohrn, A. Zur Kenntnis des Baues von Paranthura costana, Z.W.Z. 20: 81-93.

1891 Dollfus, A. Crustacés, Isopodes. Mission Scientifique du Cap Horn 1882-1893 Vol. VI, Zoologie: F. 55-F-76.

611916 Ekman, S. Systematische und tiergeographische Bemerkungen über einige glazial-marine Relikte des Kaspischen Meeres, Zool. Anz. 7: 258-269.

1935 Ekman, S. Tiergeographie des Meeres, Akad. Verlagsgesellschaft 1: 542.

1926 FEE, A. R. The Isopoda of Departure Bay and Vicinity, with descriptions of new species, varieties and colour notes, Contrib. Canadian Biol. and Fisheries 3: 13-46.

1885 Filhol, H. Crustacés in Rec. des Mémoires, Rapports et Documents relatifs à l'observation du passage dè Vénus sur le soleil Vol. 3, Pt. 2: 349-516. 
65 1881/1882 Gerstäcker, A. Isopoda, Bronn's Tierreich V, 2: 39, 244-etc.

661922 Giambiagi, D. Cuatro Isopodes de la Argentina, Physis (Rev. de la Soc. Argent. de Ciencias Naturales) 5: $230-244$.

671925 Resultatos de la Primera Expedición a Tierra del Fuego (1921) Crustacées Isopodes, Mus. Nacion H.N. Buenos Aires: $1-20$.

681929 Gurjanova, E. On the Fauna of Crustacea malacostraca of the Barentz sea, White Sea and Kara Sea, Travaux de la Soc. des Naturalistes de Leningrad 69: 29-46.

691929 - Neue Formen arktischer Isopoden und Amphipoden, Zool. Anz. 81: 309-329.

701930 - Beiträge zur Fauna der Crustacea malacostraca des Arctischen Gebietes, Zool. Anz 86: 231 - 248.

711933 - Contribution to the Isopod fauna of the Pacific Ocean I., New species of Valvifera and Flabellifera, Exploration des mers de l'U.R.S.S. Leningrad No. 17: 87-106.

721933 Die marinen Isopoden der Arktis, in Römer \& Schaudinn Fauna arctica 6: 391-470.

731935 Zur Zoogeographie der Crustacea malacostraca des arctischen Gebietes, Zoogeographica 2, Heft 4.

74 1935 Contribution tó the Zoogeography of far eastern Seas, Bull. de l'Acad. des Sciences de l'U.R.S.S. 1935: 1229-1235.

751936 Conribution to the Fauna of Crustacea malacostraca of the arctic Region, Transactions Arctic Inst. Leningrad 33: 31-44.

761936 The zoogeography of the Kara Sea, Contribution to the Fauna of Amphipoda and Isopoda of the Norhern Part of the Kara Sea, Bull. de l'Acad. des Sciences de l'U.R.S.S. Classe des Sciences mathematiques et Natur. 566-598.

771936 Beiträge zur Kenntnis der Isopodenfauna des Pazifischen Ozeans IV, Neue Isopodenarten aus dem Japanischen und Bering-Meer in Zool. Anz. 114: 250-265.

781936 Crustacés Isopodes des mers orientales, Fauna de l'U.R.S.S., Nouv, Sér. 6, Vol. VII No. 3: $1-278$.

791924 Hale, H. M. Notes on australian Crustacea II, Transact. Proceed, Roy Soc. South Australia 48: 1-6.

801924 The Crustaceans of South Australia Part I. Handbooks of the Flora and Fauna of South Austra-

lia, issued by the British Science Guild (South Australian Branch) Adelaide 1-200.

81 1924 Notes on Australian Crustacea No. 2, Transact, R. Soc. South Australia 48: $209-225$.

$821925-$ Notes on Australian Crustacea, Rec. of the South Austr. Mus. 3: 33-34.

831927 The fauna of Kangaroo Island, South Australia No. 1. The Crustacea, Transact. Roy. Soc. S. Australia 51: $307-321$. Australia, issued by the Britisch Guild (South Australian Branch) Adelaide 201-380. trium and its species, Proc. Zool. Soc. London 1904, 2: 302-331.

881916

891925

$90 \quad 1925$

The order Isopoda, the Danish Ingolf Expedition III, 5 Crustacea Malacostraca IIl: 1-262.
Studies on Arthropoda II, Kjøbenhavn Gyldendalske Boghandel 1-176.

HARGER, O. Reports on the results of dredging, under the supervision of Alex agassiz XXIII, Report on the Isopoda, Bull., Mus., Compar, Zool. Harvard College 11.

911881 Haswell, W. A. On some new Australian marine Isopoda I, Proc. Linn. Soc. N.S.W. 9: 676-679.

921882 Catalogue of the Australian stalk- and sessile-eyed Crustacea, Sydney.

931884 A revision of the Australian Isopoda, Proc. Linn. Soc. New. South Wales 9: 1001-1018.

$941884-$ On a new Crustacean found inhabiting the Tubes of Vermilia, Proc. Linn. Soc. N.S.W. 9: 676-679.

951868 Heller, C. Reise der Österreichischen Fregatte Novara um die Erde in den Jahren 1857 -1859 unter den Befehlen des Commodore B. von Wüllerstorf Urbain, Zool. Teil Bd 2, Abt. 3 Crustaceen, Vienna 1868. 
961893 Hilgendorf, F. Bemerkungen über 2 Isopoden, die japanische Süsswasser-Assel und eine neue Munnaart, Sitzber. Gesellschaft Naturf. Freunde Berlin.

971902 Hodgson, T. V. Crustacea in Report on the Collections made in the antarctic regions during the voyage of the Southern Cross London 1902. 1. 228-261.

981910 — National Antarctic Expedition, Natur. Hist. V. Crust. IX Isopoda: 1-77.

99 1936 Hult, J. On some species of Parasellidae, Arkiv for zoologi, 29A. 6; 1-11.

99 1937 Marina Isopoder från svenska Västkusten, Supplement till Wahrberg. Sveriges marina och lacustra Isopoder, Götebergs Kgl. Vet.-och Vitterhets Samh. s. Handl. 5 Fjöld (B) 5, No. 3.

1001934 JäGERSKJÖLD, L. A. Kattegats expeditionen, Göteborgs Museums Arstryck 1933/1934.

1011938 KesselyaK, A. Die Arten der Gattung Jaera, Leach (Isopoda Asellota), Zool. Jahrb. Syst. 71: 219252.

1021911 Koehler, R. Arcturides nouveaux de la campagne de la Princesse Alice, Bull. Instit. Océanographique Monaco No. 214.

1031880 Kossmann, N. Zoologische Ergebnisse einer Reise in die Küstengebiete des Rothen Meeres. Zweite Hälfte, Lief. I, Leipzig 1880.

104 1934 LovÉN, P. M. Zur Kenntniss einiger Amphipoden und Isopoden im Öresund, Unters. aus dem Öresund XVII.

1051937 Lundström, A. Neue Fundorte für Cyathura carinata (Kroyer) und Polydora ligni Wester, Kungl. Fysiografiska Sällskapets i Lund Förhandlingar 7, 15: 172-174.

1933 Moloney, J. O. Two new Isopod Crustaceans from California, Jl. Washington Acad. Sc. 23: $144-147$.

1876 Miers, E. J. Descriptions of some new species of Crustacea, chiefly from New-Zealand, Ann. Mag. Nat. Hist. (4) 17: 218-229.

1081881 Revision of the Idoteidae, a family of sessile-eyed Crustacea, Jl. Linnean Soc. 16: 1-88.

1091884 Report on the zoological collections made in the Indian Ocean during the voyage of H.M.S. Alert 1881-1882 Crustacea: 311.

1101840 Milne Edwards. H. Histoire naturelle des Crustacés Paris 1840: 1—638.

1111922 Monod, TH. Sur un essai de Classification rationelle des Isopodes, Bull. Soc. Zool. Paris 47: 134-140.

1121925 Tanaidacés et Isopodes aquatiques de l'Afrique occidentale et septentrionale, Boll. Soc. Sc. Nat. Maroc. 5: 238-241.

1131926 -_.. Les Gnatbiidae. Essai Monographique, Mém. de la Soc. de Sciences naturelles du Maroc 1926 No. 13: $1-667$.

114. 1926 - Tanaidacés, Isopodes et Amphipodes, Résultats du Voyage de la Belgica en 1897-1899, Rapports Scienfiques Zoologie 1926: 1-67.

1151927 Notes Isopodologiques II. Sur un Anthuride nouveau du Cameroen, Nothanthura barnardi nov. gen. nov. spec., Bull. Soc. Zool. France 52: 200--211.

1161931 Tanaidacés et Isopodes sub-antarctiques de la Collection Kohl-Larsen du Senckenberg Museum, Senckenbergiana 13: $10-30$.

1171931 - Sur quelques Crustacés aquatiques d'Afrique (Cameroen et Congo), Revue de Zoologie et de Botanique africaines 21: Fasc 1: 1 -36.

118 1931 Crustacés de Syrie, Extr. de A. Gruvel. Les états de Syrie, Paris. 1931.

1191933 - Tanaidacea et Isopoda. Mission Robert Dollfus en Egypte, Mém. Inst. Egypt. 21: 161—264.

1201934 - Isopodes marins des campagnes du "de Lanessan", Note Instit. Océanograph. Indochine 23: $1-22$.

1211924 Nicholls, G. E. Phreatoicus lintoni, a new species of freshwater Isopod from western Australia, J1. R. Soc. West-Australia Perth 10: 91-104.

1221927 A description of two new Genera and Species of Phreatoicidea, with a discussion of the affinities of the members of the family, Jl. Roy. Soc. Western Australia 12: 179-210.

1231929 Some new species of Stenetrium from Western Australia, Proc. Linnean Soc. New South Wales 54: $361-374$.

124 1937 On the fresh water Isoteidae of New Zealand (Crustacea, Isopoda), Ann. Mag. Nat. Hist. (10) 19: $113-135$. 
125

126

127

1281918

1291922

1301931

$131 \quad 1923$

1321930

1 33 1906

134. 1906

1351907

136

137

1935

1381886

1901

140A 1927

140B 1921

$141 \quad 1887$

142

1431921

1899

146 new species, Proc. Un. Stat. Nat. Mus. 21. 815-869.

1471904 - Contributions to the natural history of the Isopoda, Proc. U.S. State Nation. Mus. 27: 1-89.

$1481904-C o n t r i b u t i o n s$ to the natural history of the Isopoda (second part), Proc. U. S. Nation Mus. 27: $657-681$.

1491905 - A monograph on the Isopods of North America, Bull. U.S. Nat. Mus. 54: 1-727.

1501906 Isopods collected in the North West pacific by the U.S. Bureau of Fisheries Steamer Albatros in 1906, Proc. U.S. National Mus. 37: 75-120. 
1906 Richardson, H. Isopodes, Expéd. Antarctique française Jean Charcot 1903-1905 Sciences naturelles, Documents Scientifiques, Crustacés, 1906: 1-23.

152

1731900

1741900

1751914

1761936

1771927 Shepard, E. M. Revision of the Familly Pbredtoicidae (Crustacea) with a description of two new species, Proc. Zool. Soc. London: 81-124.

1781937 SpÄrcK, R. The benthonic animal communities of the coastal waters of Iceland, Zoology of Iceland I Pt. 6. ments Scientifiques 2ème mémoire 1908.

- Some new Isopoda of the Superfamily Aselloidea from the Atlantic Coast of North America, Proc. U. Stat. Nation. Mus. 35: 71-86. Nat. Mus. 34: 67-69.

- Description of a new Isopod of the Genus Eurycope from Martha's Vineyeard, Proc. Unit. States

Isopods of the North Western Pacific Albatros 1906, Proc. U.S. Nat. Mus. 37: 75-129. $421-422$.

Marine Isopods collected in the Phillippines by the U.S. Fisheries Steamer Albatross in 19071908, Department Commerce Lab. Bureau of Fisheries Doc, 736 Washington: 1-44.

— Les Crustacés Isopodes du Travailleur dt du Talisman. Bull. Mus. Paris: 1911: No. 7: 518—534.

from from the Mouth of the Rio de la Plata, Argentine, South America Proc. U.St. Nat. Mus. 40: $169-171$.

States Nat. Mus. 40: 633-635.

1912 - Isopodes du Sandwich du Sud, Museo Nacional de Buenos Aires 14: 395—400.

1912 Descriptions of two new Isopods, an Apseudes and a Munnopsis, both from the Galapagos islands, Proc. U.S. National Mus. 43: 159-162.

- Crustacés isopodes 2ème expédition antarctique française 1908-1910, Paris 1913, 24 pp.

Tropical Pacific. Albatross 1904-1905 XXVIII Isopoda, Bull. Mus. Comp. Zool. Harvard, Coll. Cambridge Mus. 58: 361--372.

St. Lawrence and Davis Straits, Proc. Royal Soc. Edinburgh 20: 154-163.

AEMUndsson, B. Icelandic Malacostraca in the Museum of Reykjavik, Rit. Visindafélags Islendinga Reykjavik.

-1886 Sars, G. O. Crustacea I-II Den Norske Nordhavs-Expedition, 1876-1878 Zoologi.

An Account of the Crustacen of Norway Vol ii, Isopoda: 1-270.

- Pacifisches Planton. Crustaceen, Zool. Jhrb. Syst. 21: 371-414.

\section{2: $122-138$.} toria, New Ser. 13: 25-28.

- Janirella, a new Genus of Isopoda from Freshwater, Victoria, Proc. Roy. Soc. Victoria New Ser. 13: $124-130$.

Sexton, E. W. On Antbura gracilis (Montagu), Jl. marine biol. Assoc. 10: 236-243.

SheArd, K. A new Phreatocoid from the Grampians, Victoria, Records of the South Australian Museum 5: 469-473.

Penger, B. \& Hall, T. S. On a new Genus of terrestrial Isopoda (Phreatoicopsis), Proc. Roy, Soc. Victoria, N. Ser 9: 12-20. 
